\begin{abstract}
Title of Document:

A CRITICAL PARAMETER OPTIMIZATION OF LAUNCH VEHICLE COSTS

Todd Herrmann

Master of Science, 2006

Directed By:

Associate Professor David Akin

Department of Aerospace Engineering
\end{abstract}

A parametric analysis aimed at minimizing costs of payload to low earth orbit is undertaken. By identifying a range of payload sizes, the effects of manipulating a number of critical parameters involving vehicle configurations on payload costs were examined. Vehicle configurations encompass single stage and multistage vehicles with combinations of airbreathing and/or rocket propulsion systems. Launch systems could be expendable or reusable on a stage-by-stage basis. Staging velocity is optimized for minimum cost at each design point. The costing model includes the effects of learning on production and operations, discount factors for multiyear investments, and the use of "refurbishment fraction" (fraction of initial procurement costs required for reusable vehicle refurbishment between flights) for estimating maintenance and turn-around costs. Overall vehicle recurring and nonrecurring costs are estimated based on sets of inert-mass cost estimating relations drawn from published sources. 


\section{A CRITICAL PARAMETER OPTIMIZATION OF LAUNCH VEHICLE COSTS}

By

Todd Matthew Herrmann

Thesis submitted to the Faculty of the Graduate School of the University of Maryland, College Park, in partial fulfillment of the requirements for the degree of

Master of Science

2006

Advisory Committee:

Associate Professor David Akin

Professor Norman Wereley

Dr. Mary Bowden 
(C) Copyright by

University of Maryland Space Systems Laboratory

2006 


\section{Acknowledgements}

This work was supported by the Space Vehicle Technology Institute under grant NCC3-989 jointly funded by NASA and DOD within the NASA Constellation University Institutes Project, with Claudia Meyer as the project manager.

Thanks to my advisor Dave Akin, for providing me with the opportunity to do this work. Thanks to the students and staff at the SSL for being so supportive and creating and being part of such a unique learning environment.

Thanks to Carolyn, who has made me so happy with her unconditional love and support.

Finally thanks to my parents who from an early age have instilled in me the importance of a broad education. I wouldn't have made it here without them. 


\section{Table of Contents}

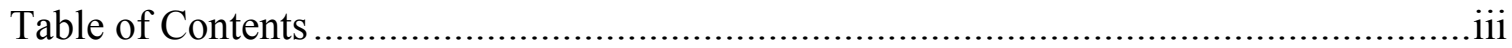

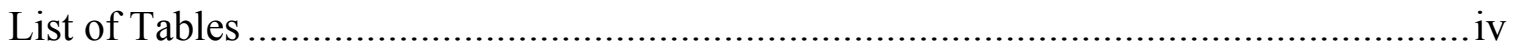

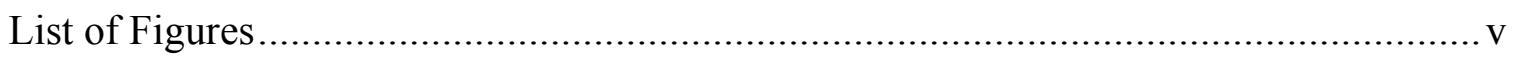

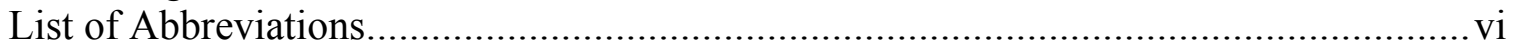

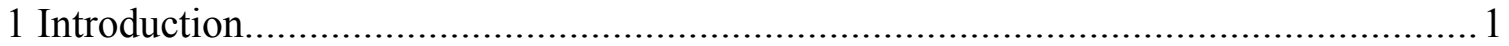

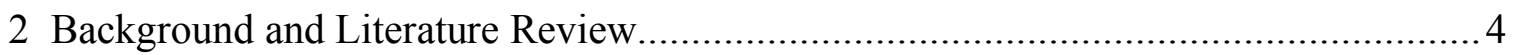

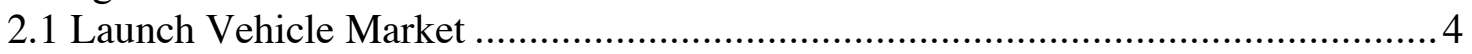

2.2 Launch Vehicle Cost Modeling....................................................................... 7

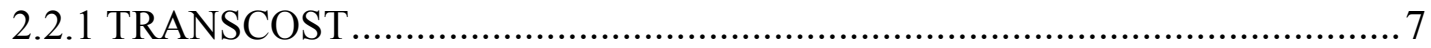

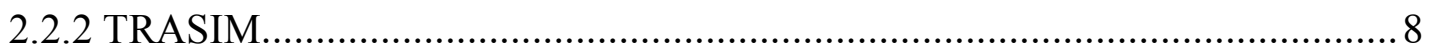

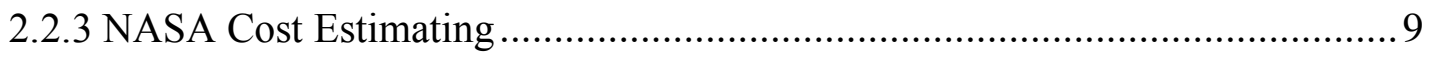

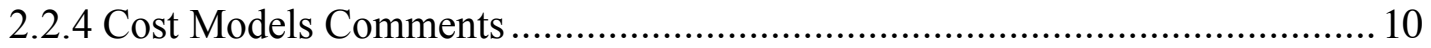

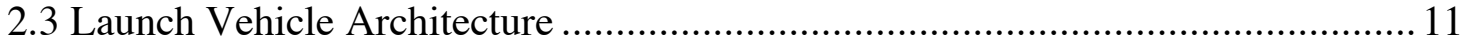

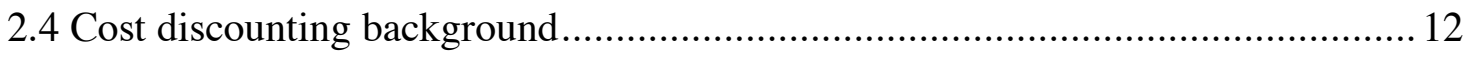

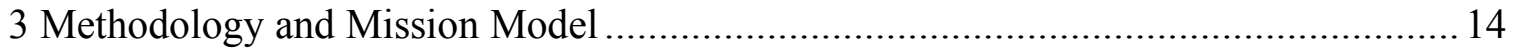

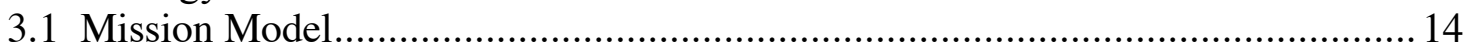

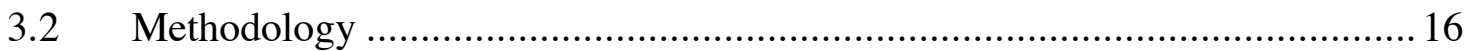

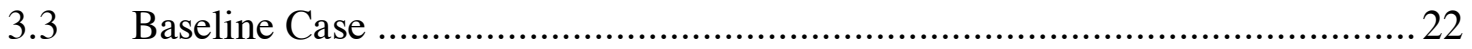

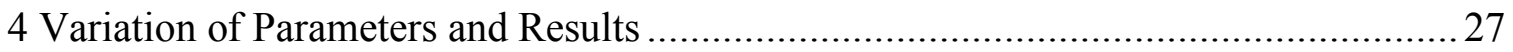

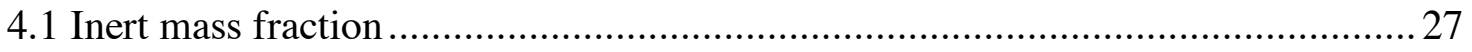

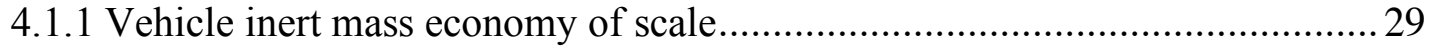

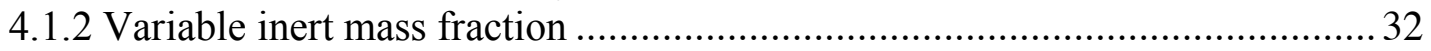

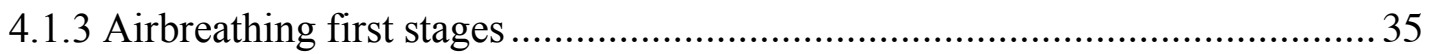

4.2 Refurbishment fraction and number of flights per vehicle ..................................... 38

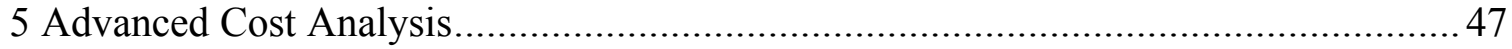

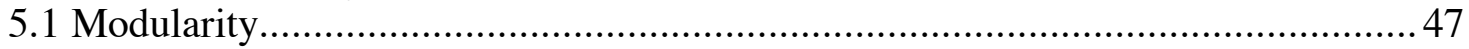

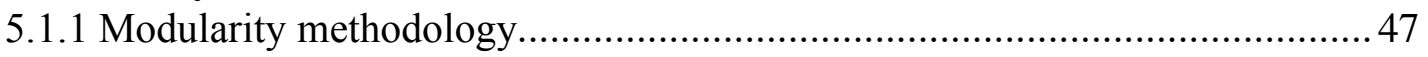

5.1.2 Inert mass fraction and operations cost complexity factors ............................52

5.1.2.1 Inert mass fraction complexity factors ...................................................5 53

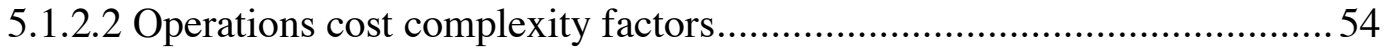

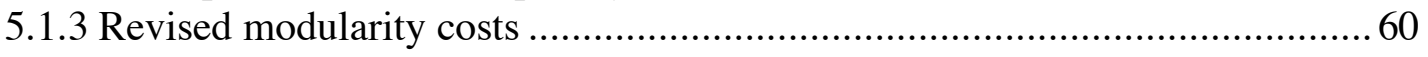

5.1.4 Expendable Launch Vehicles with Modularity ................................................63

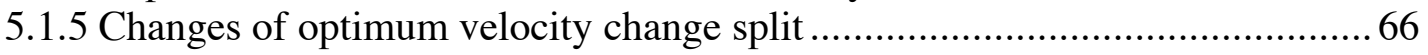

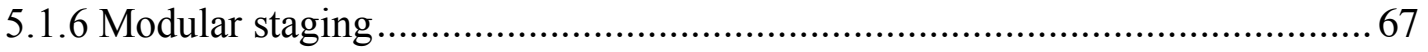

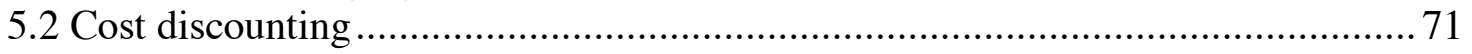

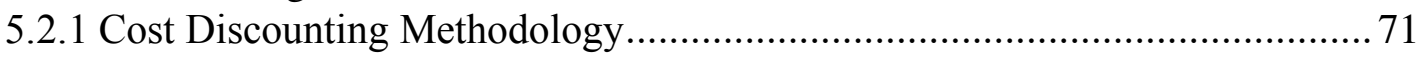

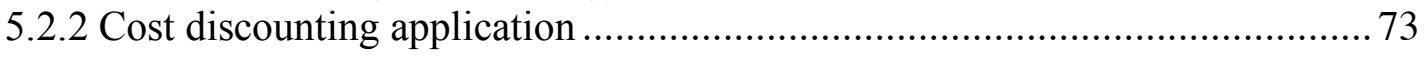

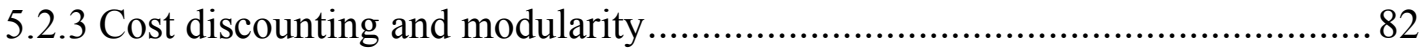

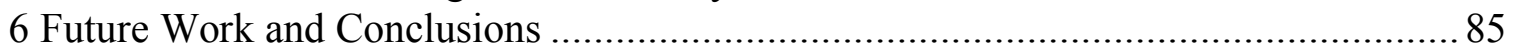

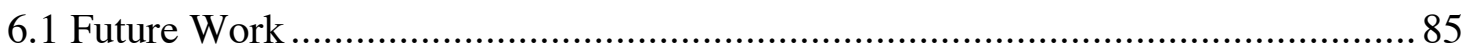

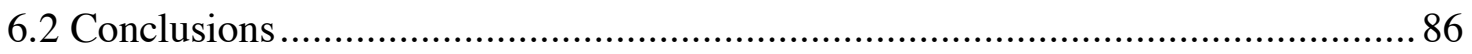

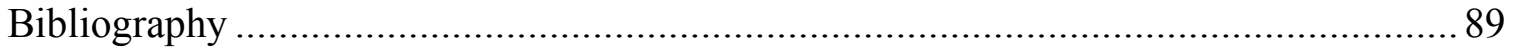




\section{List of Tables}

Table 4.1: Inert mass fractions of air breathing vehicles ........................................... 35

Table 4.2: Refurbishment fraction and flights per vehicle at $\delta=0.12$ and $I_{s p}=450 \ldots \ldots \ldots .42$

Table 5.1: Inert mass fraction as a function of number of modules................................54

Table 5.2: Operations complexity factor as a function of number of modules ...............55

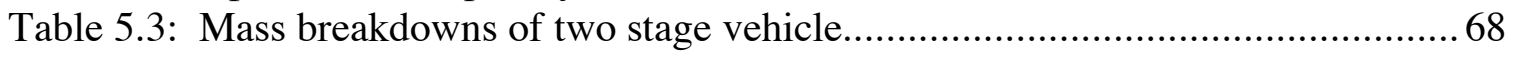

Table 5.4: Optimal dv values for stage 1 modules............................................. 68

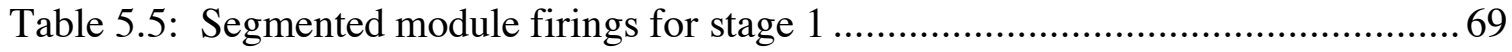

Table 5.6: Costs and revenue at 50\% return rate .................................................... 74

Table 5.7: Solved for breakeven costs and revenue at 50\% return rate ......................... 76

Table 5.8: Payload values at minimum cost for various rates of return........................ 76 


\section{List of Figures}

Figure 2.1: Launches by type last five years .................................................. 5

Figure 2.2: Ten year forecast for commercial launches ...................................... 6

Figure 3.1: Baseline Vehicle Payload Costs............................................................ 23

Figure 3.2: Baseline Vehicle Development Costs ................................................... 24

Figure 3.3: Baseline Vehicle 1st Unit Costs............................................................. 24

Figure 3.4: Baseline Vehicle Production Costs .......................................................... 25

Figure 3.5: Baseline Vehicle Operations Costs ..................................................... 25

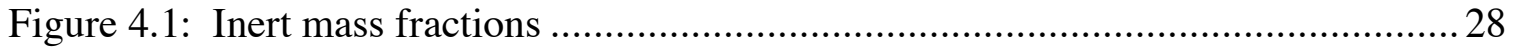

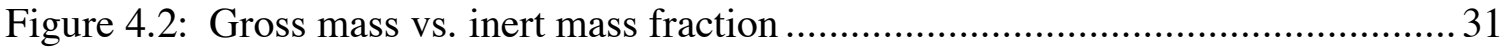

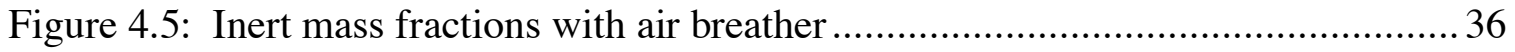

Figure 4.6: Airbreathing Staging Mach 5-14....................................................... 38

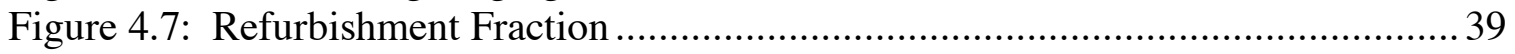

Figure 4.8: Refurbishment fraction corrected for RLV vs. ELV .............................. 41

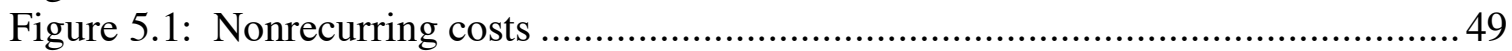

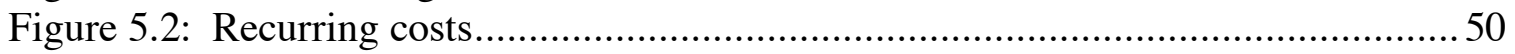

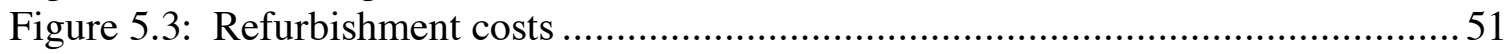

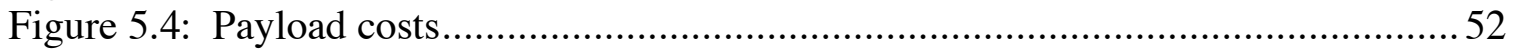

Figure 5.5: Operations cost multiplier (number of modules) …............................56

Figure 5.6: Operations cost multiplier (square root number of modules) ......................56

Figure 5.7: Operations cost multiplier (third root number of modules) .........................57

Figure 5.8: Operations cost multiplier (fourth root number of modules) ......................57

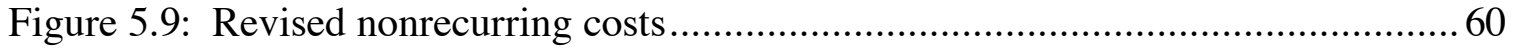

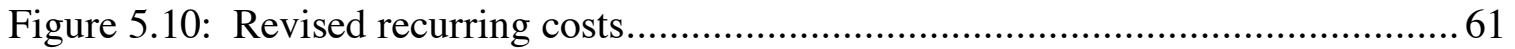

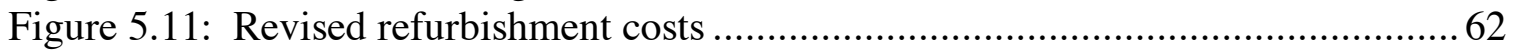

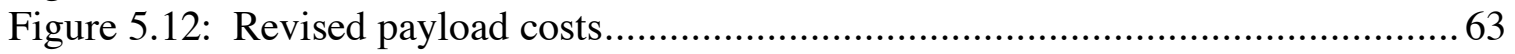

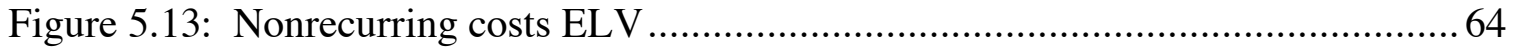

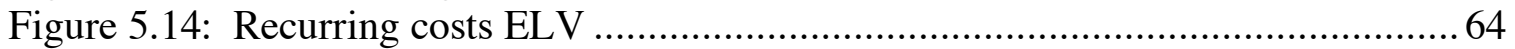

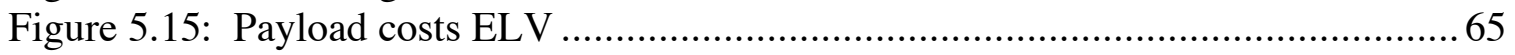

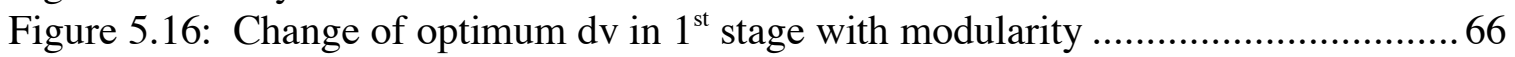

Figure 5.17: Revenue required at various rates of return for small refurbishment fraction

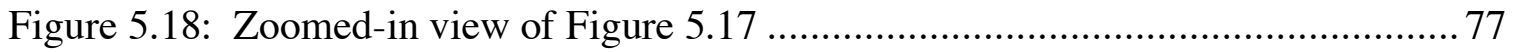

Figure 5.19: Revenue required at various rates of return for large refurbishment fraction

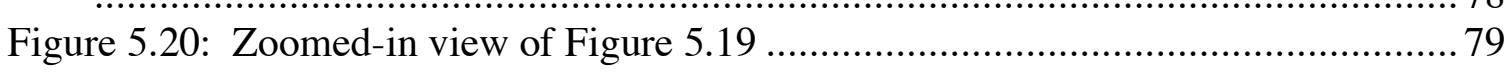

Figure 5.21: Revenue required at various rates of return for baseline ELV ...................8 80

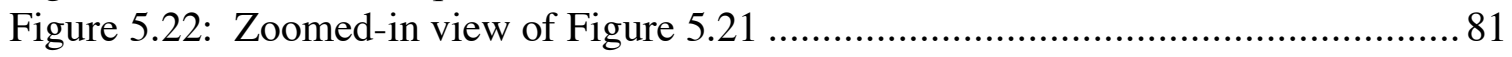

Figure 5.23: Modularity applied to discounting at 20,000 kg payload ........................ 83 


\section{List of Abbreviations}

\begin{tabular}{|c|c|}
\hline$c_{e f f}$ & $=$ effective exhaust velocity \\
\hline$C_{o p s}$ & $=$ operations cost \\
\hline$C_{n r}$ & $=$ non-recurring cost \\
\hline$C_{\text {refurb }}$ & $=$ refurbishment cost \\
\hline$C_{r t o t}$ & $=$ recurring cost \\
\hline$C_{\text {lst unit }}$ & $=$ first unit production cost \\
\hline$C_{\$ / k g}$ & $=$ cost per kilogram payload \\
\hline$C E R$ & $=$ cost estimating relationship \\
\hline$\Delta V$ & $=$ orbital velocity \\
\hline$\delta$ & $=$ inert mass fraction \\
\hline$E L V$ & $=$ expendable launch vehicle \\
\hline EELV & $=$ evolved expendable launch vehicle \\
\hline$f_{\text {refurb }}$ & $=$ refurbishment fraction \\
\hline$g_{0}$ & $=$ acceleration of gravity $\left(9.8066 \mathrm{~m} / \mathrm{s}^{2}\right)$ \\
\hline$G E O$ & $=$ geostationary orbit \\
\hline HTHL & $=$ horizontal takeoff/horizontal landing \\
\hline$I_{s p}$ & $=$ specific impulse \\
\hline LEO & $=$ low Earth orbit \\
\hline L1 & $=\mathrm{L} 1$ Lagrange point \\
\hline L2 & $=$ L2 Lagrange point \\
\hline$M_{\text {gross }}$ & $=$ vehicle gross mass \\
\hline$M_{f}$ & $=$ final vehicle mass after takeoff \\
\hline$M_{\text {inert }}$ & $=$ vehicle inert mass \\
\hline$M_{p L}$ & $=$ payload mass \\
\hline$M_{t o t}$ & $=$ total program mass \\
\hline$M_{0}$ & $=$ initial vehicle mass before takeoff \\
\hline Mod & $=$ module \\
\hline$N_{f l t s}$ & $=$ number of flights in program \\
\hline$N_{f p v}$ & $=$ number of flights per vehicle \\
\hline$N_{v e h}$ & $=$ number of vehicles in program \\
\hline$p_{\exp }$ & $=$ learning curve factor \\
\hline$r$ & mass ratio \\
\hline$R L V$ & $=$ reusable launch vehicle \\
\hline$R B C C$ & $=$ rocket based combined cycle \\
\hline SSTO & $=$ single stage to orbit \\
\hline$S V L C M$ & $=$ spacecraft $/$ vehicle level costing model \\
\hline$T B C C$ & turbine based combined cycle \\
\hline TSTO & stage to orbit \\
\hline
\end{tabular}




\section{Introduction}

The Space Shuttle was originally "sold" as a means of achieving an order-ofmagnitude reduction in launch costs to low earth orbit. More than a hundred flights later, no significant cost savings have been evident. During the intervening two decades, a number of reusable launch vehicle projects were initiated with the hopes of substantial cost reduction, and then abandoned in the face of enormous and escalating technical challenges and development costs. In recent years, NASA and the DOD seem to have settled on evolved expendable launch vehicles (EELVs) for operational launches, but this decision is being revisited in light of the new focus on extended human exploration of the moon and Mars. Currently NASA is rejecting EELVs in favor of new Shuttle derived launch vehicles. Due to these developments, it is important to re-examine the critical design choices in next-generation Earth launch systems. The goal in this study is to develop parametric models for launch vehicle performance and costing, and to apply the parameters equally to all potential systems, thus starting from a "level playing field" for the purposes of finding the most advantageous paradigms for creating a minimum cost launch vehicle.

In recent years a paradigm shift has occurred in the approach to launch vehicle design. In the early years of space exploration, demonstrating the technological capacity to get to space and setting up a grand national image were the main motivations behind design decisions. Recently, however, launch vehicle development has become less focused on national prestige and more focused on commercialization and international 
competition for satellite launches [1]. Instead of advancing technology, cost is now the main driver behind launch vehicle development. For a launch vehicle with new advanced technology to be successful, it must be proven to offer a cost advantage.

The standard vehicle for unmanned space launches is presently multiple stage expendable launch vehicles (ELV). Many studies have examined the use of reusable launch vehicles (RLV). $[2,5,6,8,9,10]$ The original design for the Space Shuttle called for it to be fully reusable as opposed to the partially reusable vehicle the Shuttle is today. Many design concepts for RLVs, such as the X33, have been abandoned in recent years due to high development costs stemming from technical difficulties. [19]

The objective of this research is to examine traditional design variables for launch vehicle stages and their effect on cost of payload to orbit. These variables are then manipulated to represent various vehicle architectures such as ELVs, RLVs, single stage, multiple stages, various rocket propellants, and airbreathing propulsion engines. In addition to design variables that represent the physical features of a launch vehicle, costing variables representing opportunity costs over time are examined. The various vehicle design cases are compared against a baseline case of a traditional ELV with design variable values approximating ELVs in use in the launch market currently. The manipulated cases are compared to this baseline case, and the results determine which design and costing variables are key parameters in controlling payload costs of launch vehicles.

A discussion of the launch vehicle market and previous launch vehicle cost modeling is presented in Chapter 2. Chapter 3 lays out the methodology and mission model assumptions used in this study. The assembly of the launch vehicle costing 
database, identification of critical parameters and variables for minimizing cost and the value selection criteria of those variables is also presented. Analysis and the effects of manipulation of the critical parameters on various launch vehicle types are discussed in Chapter 4. Chapter 5 presents the concepts of breaking launch vehicle stages into multiple modules as opposed to a monolithic stage to minimize costs. The effects of cost discounting for a new launch vehicle program paid for by commercial or private investors are also covered in this section. Chapter 6 concludes this phase of the research and offers suggestions for future work. 


\section{Background and Literature Review}

\subsection{Launch Vehicle Market}

In the late 1990's rapid growth was also in the launch vehicle market as telecommunications and related technologies experienced rapid expansion. A number of LEO satellite constellations such as Iridium, Globalstar and ORBCOMM were proposed. In 1998, the FAA projected the launch of over 1,000 satellites in the next decade to nongeosynchronous orbits [2]. To support this high rate of projected launches, a number of reusable launch vehicle concepts were proposed such as Lockheed-Martin's VentureStar. However, the operators of these new LEO satellite constellations suffered financial setbacks before their systems came to fruition, and consequently the expanding market for reusable launch vehicle concepts disappeared before their development was complete. In 2001, the FAA forecast only 151 satellites in the next decade. This represented an $80 \%$ cut in the expected market of the new reusable vehicles.

The current launch industry is fairly stable at 50-70 launches per year [3]. The total world launch market in the last five years, both commercial and non-commercial, is shown in Figure 2.1. 


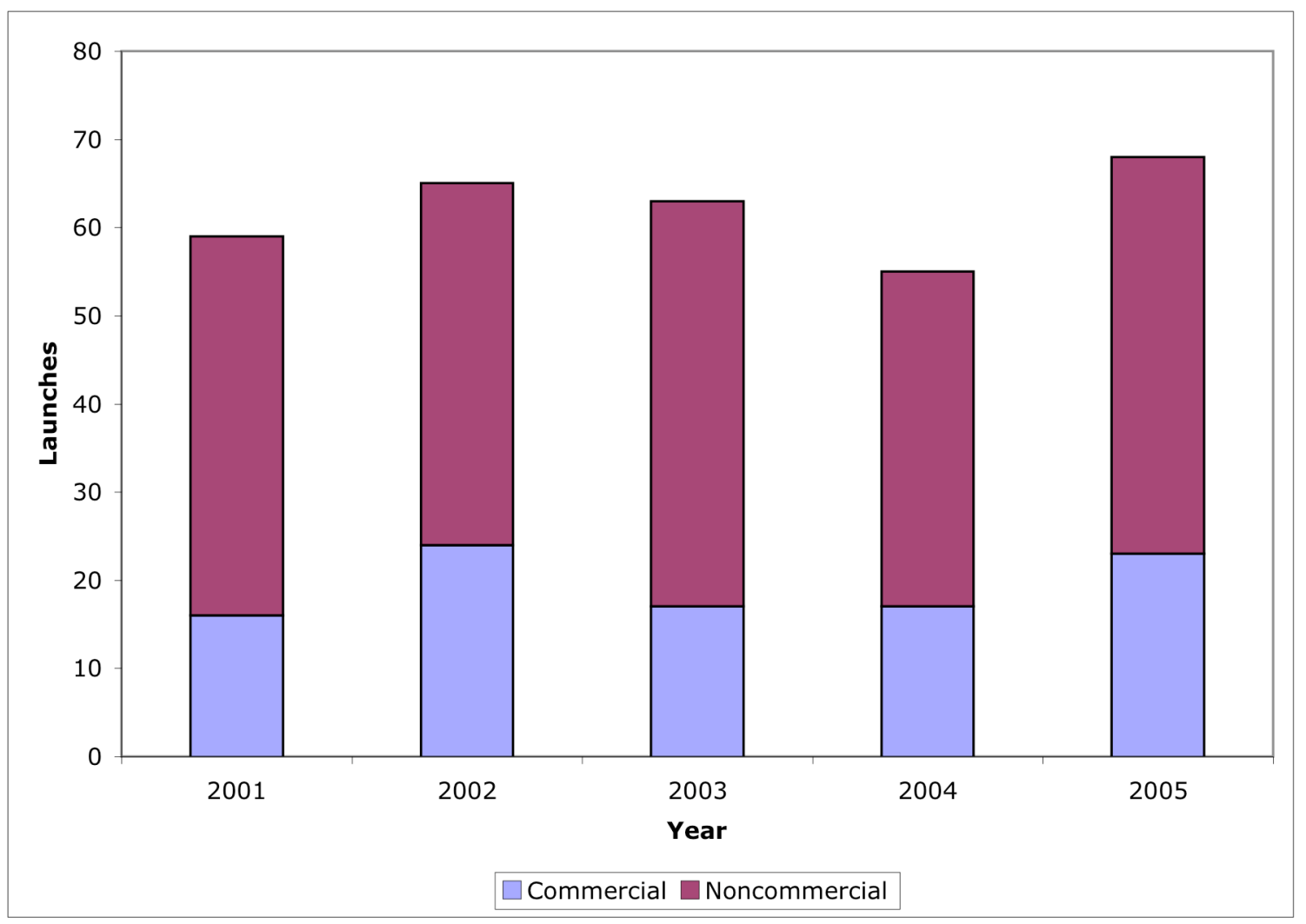

Figure 2.1: Total launches 2001-2005

Data from Futron Corporation [3]

A significant majority of these launches are non-commercial launches primarily made by the governments of the USA, Russia and China. The leaders in the commercial launch market are the USA, Russia and Europe.

The FAA Commercial Space Transportation Forecast [4] predicts the number of commercial launches to decline over the next ten years and then stay fairly constant around 20-25 launches per year with a clear majority of launches sending satellites to GSO as shown in Figure 2.2. 


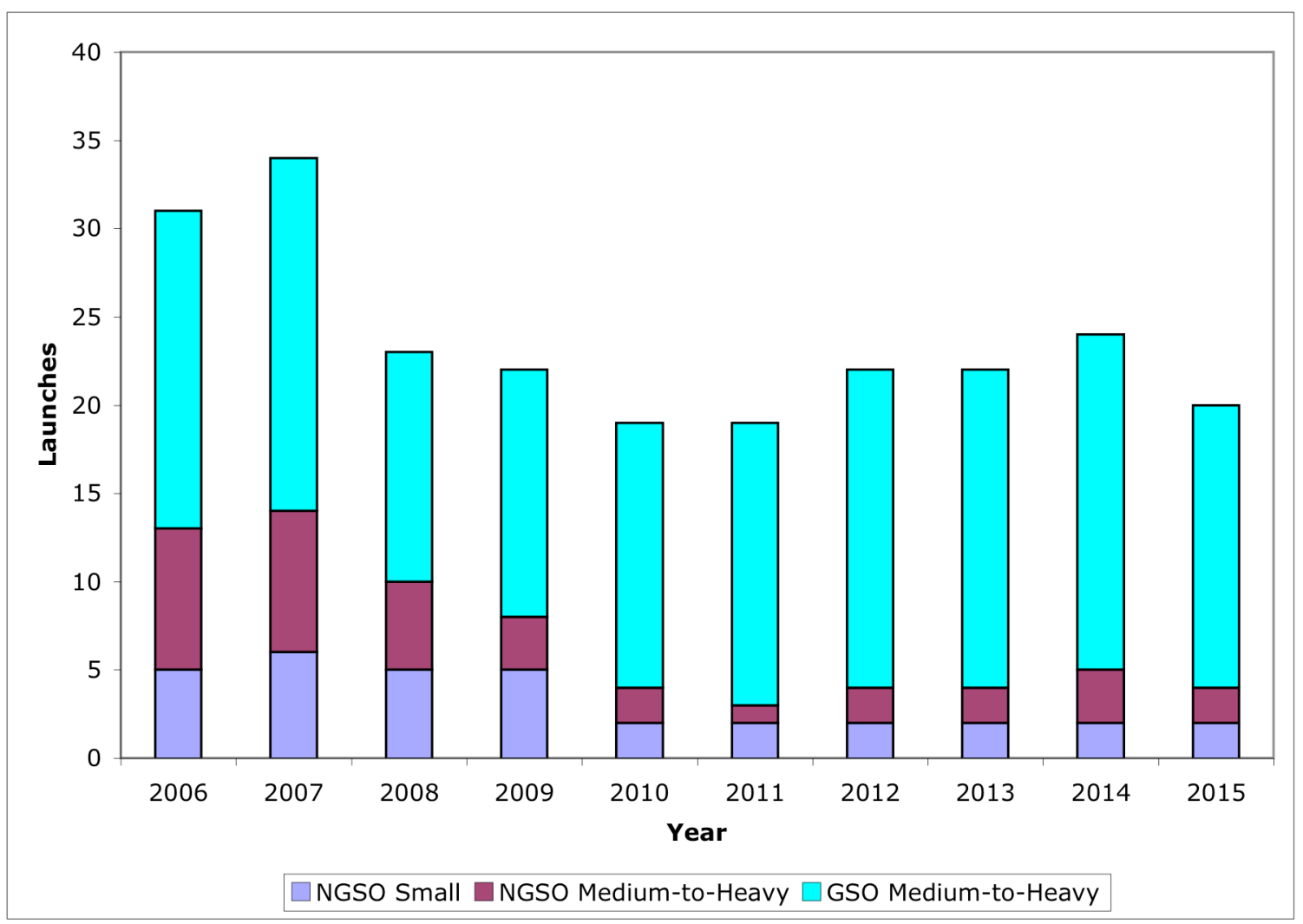

Figure 2.2: Ten year forecast for commercial launches Data from FAA [4]

However, some of the companies operating LEO satellite systems have emerged from bankruptcy in recent years. ORBCOMM and Globalstar both have plans to launch replenishment satellites over the next few years. This will give a minor boost to the number of launches to LEO in the near future.

This research assumes a fairly active launch market where demand for launch capabilities to LEO is high. It has been shown that for a fleet of new RLVs to be economically successful, the launch rate must be high; economic viability also requires heavy commercial development with cost amortization [1]. The effects of commercial financing of new launch vehicle programs at various rates of return are examined in Chapter 5. 


\subsection{Launch Vehicle Cost Modeling}

Cost modeling is an important aspect of the development and design process of launch vehicle programs. Many new programs that showed great potential on the drawing board, such as the $\mathrm{X} 33$, ultimately failed in part due to cost overruns. It is vital to the success of new launch vehicle programs to accurately cost new technology and to prove that it is cost effective. Government, military and academia have developed a number of cost models for this purpose. Many of these models are based on a set of heuristic equations developed by examining launch vehicle data from vehicles that have already been designed, developed and flown. Two significant non-government costs models (TRANSCOST and TRASIM) are summarized below, followed by cost models developed by NASA and the U.S. military.

\subsubsection{TRANSCOST}

Dietrich E. Koelle wrote the TRANSCOST "Statistical Analytical Model for Cost Estimation and Economical Optimization of Space Transportation Systems" [5]. It uses historical data from an international database of space vehicles and engine projects since 1960 to document actual costs of completed projects through statistical methods. These findings are then used to extrapolate future projects with advanced technology such as ballistic reusable and winged orbital vehicles. This model is used for the design phase of new space vehicles and engines with a systems level view and minimal emphasis on subsystems. The three main cost categories covered in TRANSCOST are development cost, recurring cost and ground and flight operations cost. Development and recurring 
costs are divided into cost estimating relationships (CERs) for engines and vehicle systems. Engines covered are solid propellant, liquid propellant, pressure-fed and airbreathing turbo and ramjet engines. Vehicle systems detailed are propulsion modules, expendable ballistic stages, reusable ballistic stages, winged orbital vehicles, high-speed aircraft and winged first stage vehicles and crewed space vehicles. Most of these CERs are derived based on vehicle or component mass and take the form:

$$
C=a \cdot M^{x}
$$

where $C=$ cost, $a=$ a system specific constant, $M=$ vehicle or component mass in $\mathrm{kg}$ and $x=$ a system specific cost to mass sensitivity factor. Many of these CERs also use a series of factors which are multipliers to the basic CER to reflect a system's specific level of technical development, technical quality, team experience or learning for programs with series production. The costing unit here is the "Man-Year" which is defined as the "total industrial project cost divided by the number of fully accounted people." The advantage of using the Man-Year as a costing unit is that it is independent from annual changes due to inflation and international currency exchange rates. Based on historical cost data regression analysis, Koelle shows that TRANSCOST is accurate to a range of $+/-15$ to $25 \%$.

\subsubsection{TRASIM}

H.H. Koelle and B. Johenning developed TRASIM (Space Transportation Simulation Model) [6]. TRASIM simulates the life cycle of a fleet of space transportation systems broken down on year-by-year basis. The difference between TRASIM and

TRANSCOST is that TRASIM performs cost estimations at the subsystem level and 
simulates the entire life cycle of the fleet broken down on an annual basis. Also while TRANSCOST focuses mainly on missions to LEO and GEO, touching briefly on lunar and interplanetary missions, TRASIM considers mission models between nine different locations in the solar system. These include Earth's surface, LEO, GEO, L1, L2, lunar orbit, lunar surface, planetary escape vector, and Mars surface.

\subsubsection{NASA Cost Estimating}

There are several cost estimating tools used by NASA's Cost Estimating and Assessment Office (CEAO). The CEAO operates from JSC and has simple cost estimating tools for a variety of air and space transportation systems available at their website [7]. These models are designed for quick estimation of the development and production cost of a vehicle, launch stage or component based on simple design parameters such as dry mass, number of vehicles to be produced, learning curve, speed (for air vehicles) and complexity. For the basis of this study, the Spacecraft/Vehicle Level Costing Model is used. The SVLCM is a top-level model derived from the NASA/Air Force Cost Model (NAFCOM) database. NAFCOM was developed at Marshall Space Flight Center beginning in 1990 in response to the need for a standardized cost-estimating tool for NASA. NAFCOM consolidates existing cost models and databases used by NASA, presenting them in software with a spreadsheet interface. Much of the information in NAFCOM comes from the Resource Data Storage and Retrieval (REDSTAR) library, which houses NASA's cost, technical, and program data back to the 1960 s. The CERs in NAFCOM are based on analogous points and averages of data in systems comparable to the one being considered. To create a new 
CER, users can select from specific vehicle type databases (e.g., liquid rocket engine, manned spacecraft, unmanned planetary spacecraft, launch vehicle stage, scientific instrument) and then the appropriate data level (group, subsystem, component, unit). Within each data level there are subcategories for groups such as structures, thermal, power, and data handling, for subsystems such as attitude control and thermal shields and for components such as batteries and wires. Users then select a series of filters to reflect technical and program considerations and enter weights and complexity factors based on the type of mission.

\subsubsection{Cost Models Comments}

One common feature of all these models is that their CERs are based on the amount of dry, or inert mass, in a vehicle. Koelle contends that inert mass fraction reduces as gross mass increases [5]. This inert mass economy of scale concept is studied further in Chapter 4 by examining historic launch vehicle inert mass fractions. Since nonrecurring, or development costs, are a function of inert mass, a declining inert mass fraction as gross mass increased would seem to give a cost advantage to larger vehicles. This is not always the case though for reusable vehicles, especially if there is commercial financing involved in their development. When factors such as the cost of refurbishment, cost discounting and the number of units produced are taken into account, it becomes clear that bigger is not always better when it comes to launch vehicles. This research examines these concepts and presents new findings on the effects of these parameters on overall program costs. 
However whenever dealing with the development of new launch vehicles, using the most advanced technology is not always the best way to cut costs. Koelle [8] states that advanced high cost materials will lower the mass of a vehicle, but drive up development costs. Using already developed hardware will cut down on development costs, and increase the gross mass of the vehicle. When any new launch vehicle program is considered, trade offs of new technology vs. additional development cost must be done. Technology that may drive up development costs could enable the vehicle to fly further and faster. Vehicle gross mass is not a major cost driver. Koelle [8] states payload capability grows faster with increasing vehicle size than development cost. This thesis research examines the effects of payload size on vehicle costs. A large range of payload sizes, from small to very large, is considered to determine the optimum vehicle size for minimizing launch costs.

\subsection{Launch Vehicle Architecture}

The architecture of a launch vehicle must also be considered when determining cost optimum design solutions. It is generally assumed that a reusable launch vehicle will reduce payload costs to orbit because the recurring costs are lower as opposed to an expendable vehicle. However, new technology costs can drive development costs of reusable vehicles up enough to overcome these cost savings. Wertz [9] states, "expendable launch vehicles will continue to have a significant economic advantage over reusable vehicles until launch rates increase by well over 100 times." It takes launch rates this high for the amortized development costs of a reusable launch vehicle to "catch up" to the cost benefits seen on the learning curve for a similar number of expendable 
launch vehicles. High launch rates are needed to amortize the cost over multiple flights sufficiently to drive down launch costs. As previously mentioned, cost effectiveness must be the driving factor behind launch vehicle development.

\subsection{Cost discounting background}

Net Present Value (NPV) compares the value of a dollar today to the value of the same dollar in the future, accounting for inflation and returns. The definition of net present value is the difference between the present value of cash inflows and the present value of cash outflows.

$N P V=\sum_{t=1}^{T} C_{t}(1+r)^{-t}-C_{0}$

In this research present value, the amount that a future sum of money is worth today given a rate of return, is used

$C_{i}=C_{i+n}(1+r)^{-n}$

The discount rate, $r$, in the above equation is the interest rate used in determining the present value of future cash flows. For example, a client will pay $\$ 1000$ in a year. That $\$ 1000$ (assuming a discount rate of $10 \%$ ) is worth $1000^{*}(1+0.1)^{\wedge}-1$ or $\$ 909.09$ today. If a client pays $\$ 1000$ in two years it is worth $1000^{*}(1+0.1)^{\wedge}-2$ or $\$ 826.44$ today. From this standpoint it would be better to get paid the $\$ 1000$ now. From the client's standpoint it is better to pay the same amount later. Deferring expenditures until later is preferable to making payments in the early years of launch vehicle programs.

No in depth analysis exists in the literature on the effects of cost discounting at various rates of return on vehicle sizing for optimum payload costs. Griffin and 
Claybaugh [10] note that high amortization costs "can cause the specific launch cost of the RLV to exceed those of existing systems" and "suggest that RLV development carried out according to commercial aerospace practices and procedures could substantially reduce development costs." Cost discounting incorporates the opportunity costs of investing and examines what revenue would need to be achieved to see rates of return investors would expect when considering funding a new launch vehicle program. This thesis research incorporates the time effects of money and examines the optimum vehicle payload size for investors to see a payback on their investment at various rates of return. Traditionally high nonrecurring costs have prevented the levels of return investors expect to see. Vehicle architectures will be manipulated to minimize these costs in an attempt to make return rates more favorable for investors. 


\section{Methodology and Mission Model}

\subsection{Mission Model}

The cost model developed for this analysis incorporates all of the major system and vehicle level costs associated with a fleet of new vehicles for launch to low earth orbit. Past analyses have demonstrated the critical role of total mission model in overall cost estimation [11]; for the purposes of this study, the total launch market is assumed to be 20 million kilograms of payload delivered to LEO at an altitude of 400 kilometers over a period of 20 years, unless otherwise noted. This period of 20 years accounts only for when launches are occurring and will be expanded upon when program development time is taken into account as noted later in this paper. Also, the total program payload mass of 20 million kilograms will be assumed except when changes are introduced to determine effects of a larger or smaller total program payload mass. This launch rate corresponds to 40-50 flights of shuttle/EELV-class vehicles per year, which is a modest increase over current launch rates. The cost model includes all development costs associated with designing a next generation launch vehicle, production and facilities costs for constructing and maintaining the fleet and operations costs for launching missions.

The total program payload is divided evenly between all flights. The number of flights required is determined by the payload size, and therefore ultimately by the overall size of the vehicles. The vehicle size and number of flights required to complete the program's objectives is iterated in the model to optimize for minimum total payload costs to orbit. While an expendable launch vehicle (ELV) costs less to develop, reusable launch vehicles (RLV) might be expected to cost less on a equivalent per mission basis, as post-mission refurbishment costs for RLVs are generally lower than the equivalent 
production costs of more ELVs. The primary focus of this research is on evaluating competing technologies for reusable vehicles, so costing results presented will focus specifically on reusable systems unless designated specifically as an expendable vehicle or stage.

Since multiple vehicles will be constructed, learning trends will affect production costs. A constant learning curve of $80 \%$ is used for this model: that is, the $2^{\text {nd }}$ unit will cost $80 \%$ of the $1^{\text {st }}$, the $4^{\text {th }}$ will cost $80 \%$ of the $2^{\text {nd }}$, and so on. The traditional learning curve in the aerospace industry is between $87 \%$ and 96\% [12]. In [12], Apgar, et al suggest a 95\% learning curve for less than 10 units, a 90\% learning curve for between 10 and 50 units and a $85 \%$ learning curve for greater than 50 units. Given the large total program mass to be launched, this model considers the production of hundreds of units in some cases. A learning curve of $80 \%$ is a good estimate for this research. The cost of the $\mathrm{n}^{\text {th }}$ unit can be determined using equations (1) and (2) below:

$$
\begin{gathered}
C_{n}=C_{1} n^{p_{\text {exp }}} \\
p_{\exp }=\frac{\log \left(\frac{C_{2}}{C_{1}}\right)}{\log (2)}
\end{gathered}
$$

The learning curve here is represented by the $p_{\exp }$ term. The learning curve for this vehicle program is set at $80 \%$, which corresponds to a $p_{\exp }$ value of -0.32 . The learning curve takes into account improved understanding of how to build the vehicle that comes with each subsequent unit, and lower costs associated with production lines for 
large quantities of each part of the vehicle. Sensitivity analysis is performed on several input parameters to determine the effects on the overall cost of the program. These input parameters are discussed in the methodology section in this chapter and following chapters.

The baseline vehicle examined here is a two stage to orbit (TSTO) system. It has been shown that a multistage launch vehicle approach has both physical and economic advantages [13] in lifting payload into orbit. Stages are modeled with rocket engines using several different types of rocket propellant and, when indicated, airbreathing engines. All dollar amounts are in \$2004US unless otherwise noted.

\subsection{Methodology}

Costs are estimated using the NASA Spacecraft/Vehicle Level Costing Model [7]; this is a set of heuristic equations for various cost elements based on overall vehicle parameters, such as inert mass. To minimize the cost per kilogram of payload to orbit, it is important to determine the optimum payload size in each vehicle. A range of payload sizes is selected from 1,000 to 75,000 kilograms; this covers vehicle sizes ranging from the smallest current launch vehicles to systems with payloads three times the current largest.

For each payload size, the following data is determined. The total number of flights required over the course of the 20-year flight period is determined by dividing the total program payload mass by the vehicle design payload mass for each flight.

$$
N_{f t s}=\frac{M_{t o t}}{M_{p L}}
$$


The total number of individual vehicles required for the program is then determined by dividing the total number of flights by the number of flights per vehicle. The number of flights per vehicle is a variable that can be set, changed and optimized by the user. If an expendable vehicle is considered, then the number of flights per vehicle is set to one.

$$
N_{v e h}=\frac{N_{f l t s}}{N_{f p v}}
$$

The mass ratio, $r$, is the classic measure of the rocket's design effectiveness. The mass ratio is defined as the ratio of the final mass of the vehicle after launch to the initial mass. It includes $\Delta V$ (orbital velocity), which is the amount of velocity required for the launch vehicle to travel from the Earth's surface to circular orbit at a given altitude. The required velocity to reach a circular orbital altitude of $400 \mathrm{~km}$ is assumed to be 9200 meters per second, which includes standard allocations for gravity and aerodynamic losses. The staging velocity split is represented as the fraction of the total change in velocity $(9200 \mathrm{~m} / \mathrm{s})$ at which staging occurs. For a single stage vehicle the $\Delta V$ split is one since all the change in velocity comes in the single stage. For a multistage vehicle the $\Delta V$ split for each stage is a fractional value, with all the $\Delta V$ split values adding up to one. The $\Delta V$ split between stages is optimized for each vehicle case to minimize cost. For most cases in a TSTO vehicle the optimum $\Delta V$ split to minimize cost is equal to $55-70 \%$ of the total required velocity spent in the first stage. This range is calculated using an iterative process where a best guess for the $\Delta V$ split for the first stage is entered. The cost 
per kilogram payload is computed by following equations (1) through (14) in this section. Then the $\Delta V$ split is incremented by $1 \%$ and the change in payload costs is again recorded. This iterative process continues until a minimum payload cost is achieved. The optimum $\Delta V$ split is usually on the lower end of the range, around $60 \%$, for a RLV and the higher end, around 67\%, for an ELV. Even though there is more gross mass in an RLV to account for the additional dry weight of recovery systems, the ratio of propellant mass to gross mass in both vehicles is the same. The propellant mass increases at a rate proportional to the gross mass to account for the extra propellant needed to lift a higher gross mass. However with more inert mass in RLV stages it is beneficial to the payload cost to split the required velocity change a little more evenly between stages. The optimum $\Delta V$ split may fall outside this range for special cases where major design deviations are taken from a traditional TSTO vehicle with rocket engines. Design changes such as using an airbreathing engine in the first stage or splitting the first stage into modules cause the optimum $\Delta V$ split to fall outside the 55-70\% range. These changes are further examined later in this paper.

Also required to calculate the vehicle's mass ratio is the specific impulse, $\left(I_{s p}\right)$, which is a measure of a rocket engine's efficiency and is dependent on the propellant used. Chemical propellants, which are used in the vehicles for this program, typically have a specific impulse of 200-450 seconds. $I_{s p}$ values were set in this range with specific values depending on the type of propellant used. For the airbreathing vehicles considered in this study, the (fuel) specific impulse is set at 2000 seconds. 


$$
\begin{gathered}
r=e^{\frac{-\Delta V}{c_{e f f}}}=\frac{M_{f}}{M_{0}} \\
c_{e f f}=I_{s p} g_{0}
\end{gathered}
$$

The inert mass fraction $\delta$ is one of the major vehicle estimating parameters, and is defined as the ratio of inert mass in the vehicle to the total mass of the vehicle. This formulation assumes that the total mass of the vehicle can be split into categories of payload mass, inert mass and propellant mass. The inert mass fractions used in this model are derived from historical data for vehicle configurations with past heritage, or are taken from detailed design studies for advanced technologies such as airbreathing stages. Typical values for $\delta$ range from 0.04 to 0.20 for vehicles with rocket engines and 0.20 to 0.40 for stages with airbreathing engines. The gross mass of each launch vehicle is calculated by using the payload mass and the fraction of total vehicle mass that is composed of payload mass.

$$
M_{\text {gross }}=\frac{M_{p L}}{r-\delta}
$$

The inert mass of the vehicle is determined by multiplying the inert mass fraction by the gross mass.

$$
M_{\text {inert }}=M_{\text {gross }} * \delta
$$

With the inert mass known, the costs of the launch vehicles can be determined. All the following costs are in millions of US 2004 dollars (\$M2004). Inert mass is estimated using the information from the equations above. The numerical values shown 
are constants based on the type of vehicle, in this case a launch vehicle stage, and are determined by NASA from years of launch vehicle data [7]. The numerical coefficients of these costing equations are based on data compiled in 1996. Therefore inflation must be taken into account. The last term (1.20) on the equations below represents inflation factors from 1996 to 2004 [14]. Equation (9) estimates the nonrecurring development costs for the launch vehicles.

$$
\begin{gathered}
C_{n r}=6.7 * M_{\text {inert }}^{0.55} * 1.20 \\
C_{1^{s t} \text { unit }}=0.1 * M_{\text {inert }}^{0.662} * 1.20
\end{gathered}
$$

The recurring, or production, costs for the vehicles are determined using equation (10), which estimates the production costs for the first vehicle built. Subsequent vehicle production costs are reduced due to learning effects, which tend to favor programs with larger production runs. The total program recurring cost is dependent on the production cost, the number of vehicles and the learning curve. The ideal way to account for total recurring costs would be to directly add up the estimates for each vehicle produced, which is not well suited for rapid iteration of analyses in the course of finding optimal solutions. Instead, equation (7) is an approximation for total recurring costs, which is accurate to within a few percent as long as the total number of units produced is larger than $\sim 10$.

$$
C_{r_{\text {tot }}}=C_{1^{\text {st } u n i t}} * \frac{N_{v e h}^{\left(1+p_{\exp }\right)}}{1+p_{\exp }}
$$


The vehicle refurbishment cost is dependent on the recurring cost, the refurbishment fraction, and the number of flights per vehicle. The refurbishment fraction of a vehicle $f_{\text {refurb }}$ represents the cost required for post-flight refurbishment, expressed as a fraction of the $1^{\text {st }}$ unit production cost. The refurbishment fraction's value is input by the user in this model, and based on historical data can vary from 10-20\% for a launch vehicle. The X-15 program demonstrated a 3\% refurbishment fraction over 199 flights, and that figure will be used as a lower practical limit for launch vehicle estimation.

$$
C_{\text {refurb }}=C_{r_{\text {tot }}} * f_{\text {refurb }} * N_{f p v}
$$

The operations cost is represented by a constant operations cost per flight multiplied by the total number of flights. Koelle [8] concludes that TSTO vehicles have a $20 \%$ higher operations cost than similar SSTO vehicles. Therefore operations costs are set at \$1 million per flight for SSTO vehicles and \$1.2 million per flight for TSTO vehicles in this study.

$$
C_{o p s}=f_{o p} * N_{f l t s}
$$

The total cost is determined by adding the nonrecurring, recurring, refurbishment and operations costs. Note that the $1^{\text {st }}$ unit production costs are not added separately here because all production costs are included in the recurring costs. The chosen figure of merit for this study is the cost per kilogram of payload $(\$ / \mathrm{kg})$, calculated by dividing the total cost by the total program payload mass. 


$$
C_{\$ / k g}=\frac{C_{n r}+C_{r}+C_{r e f u r b}+C_{o p s}}{M_{t o t}}
$$

Once the database has been constructed from the equations listed above, several input variables are optimized to minimize cost. The variables, $N_{f p v}, \delta, M_{p L}, \Delta V$ split and $f_{\text {refurb }}$ are user input variables in this model and can be changed. The inert mass fraction $(\delta)$ and refurbishment fraction $\left(f_{\text {refurb }}\right)$ represent limitations of technology, and are used as independent variables in the analysis. The flights per vehicle $\left(N_{f p v}\right)$ and launch vehicle payload mass $\left(M_{p L}\right)$ are optimized to find values resulting in the minimal cost per kilogram of payload to orbit.

\subsection{Baseline Case}

To fully understand the implications of manipulating the design variables discussed above it is useful to define a vehicle with baseline values of specific design variables. This provides a platform by which to compare the positive or negative effects of all other manipulations and changes of the design variables on vehicle costs. This baseline vehicle is an unmanned TSTO vehicle with cryogenic (LOX/LH2) engines in both stages. The cryogenic engine is chosen for its high fuel efficiency $\left(I_{s p}=425\right.$ seconds). Since most launches currently are using an ELV, the Shuttle is the only partially reusable vehicle in use today; the baseline case is set as an expendable vehicle. The inert mass fraction is set at $\delta=0.078$. This number is based on the average of a number of expendable stages taken from historical data [15]. Since both stages are expendable, the number of flights per vehicle is equal to one and the refurbishment 
fraction is 0 . Also the $\Delta V$ split for the first stage is set at 0.67 . This is the relative proportion of the total velocity change that should be completed during the firing of the $1^{\text {st }}$ stage to minimize the payload cost of the expendable vehicle. Cost trends in the baseline vehicle over the major cost categories are shown below.

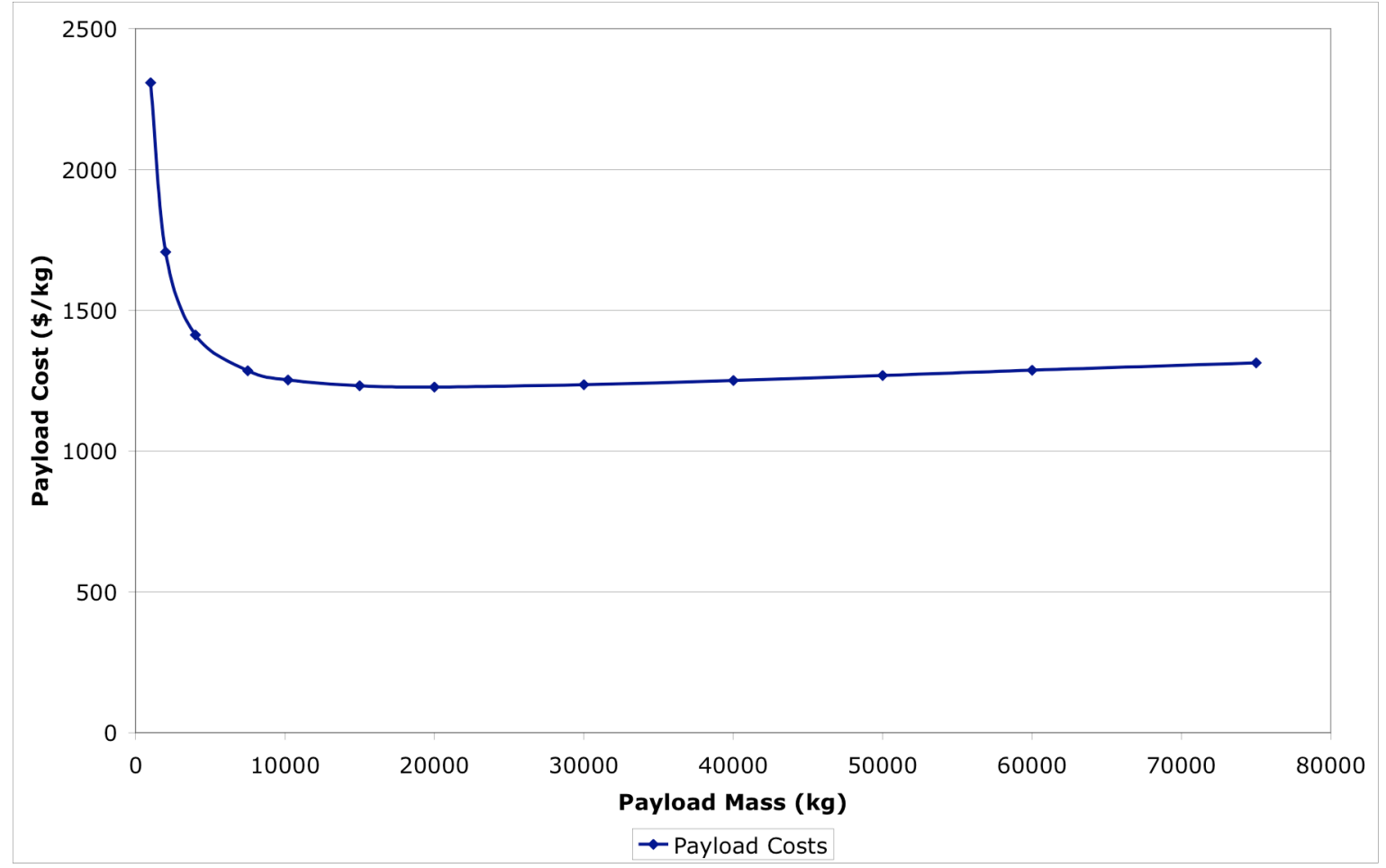

Figure 3.1: Baseline Vehicle Payload Costs 


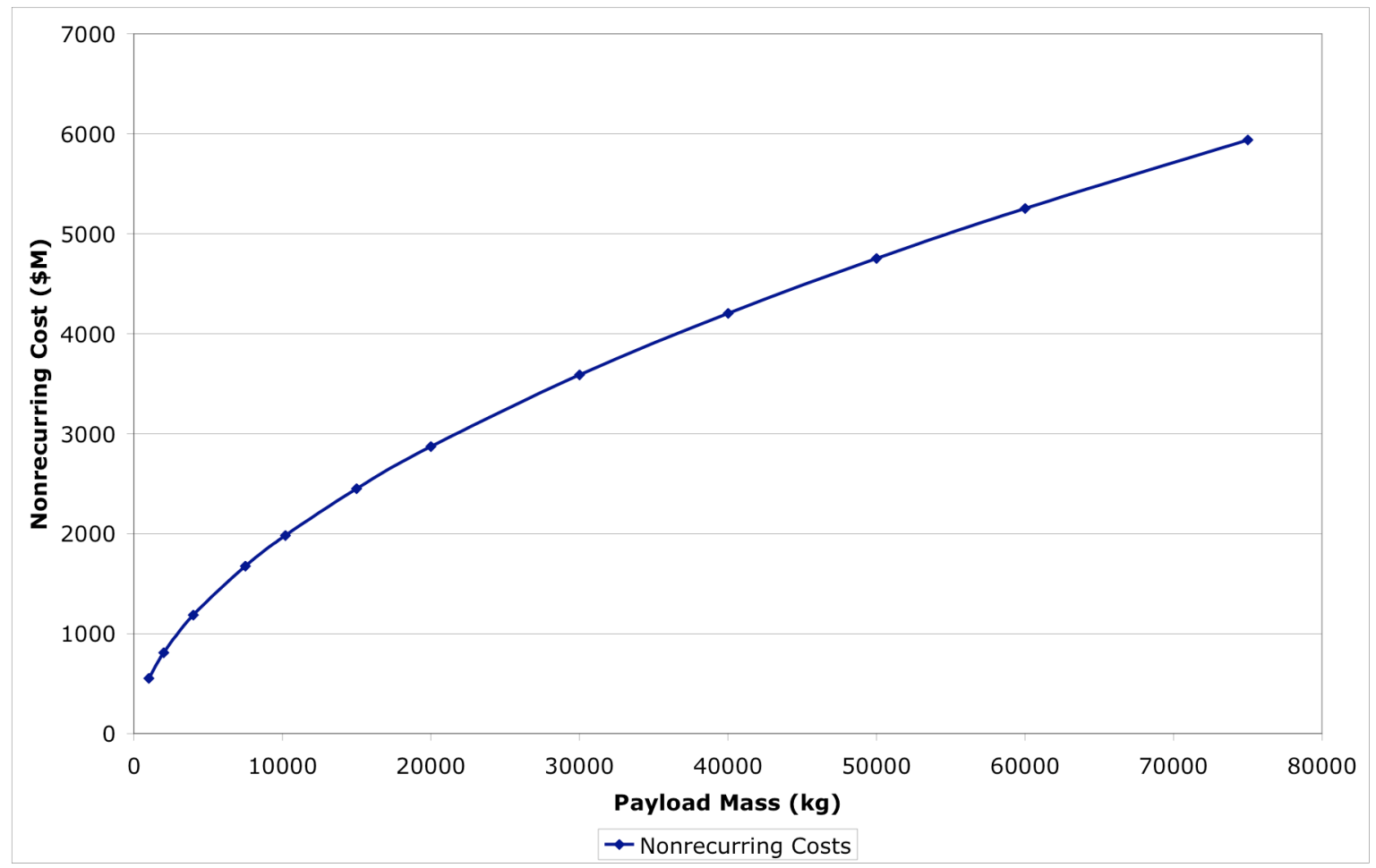

Figure 3.2: Baseline Vehicle Development Costs

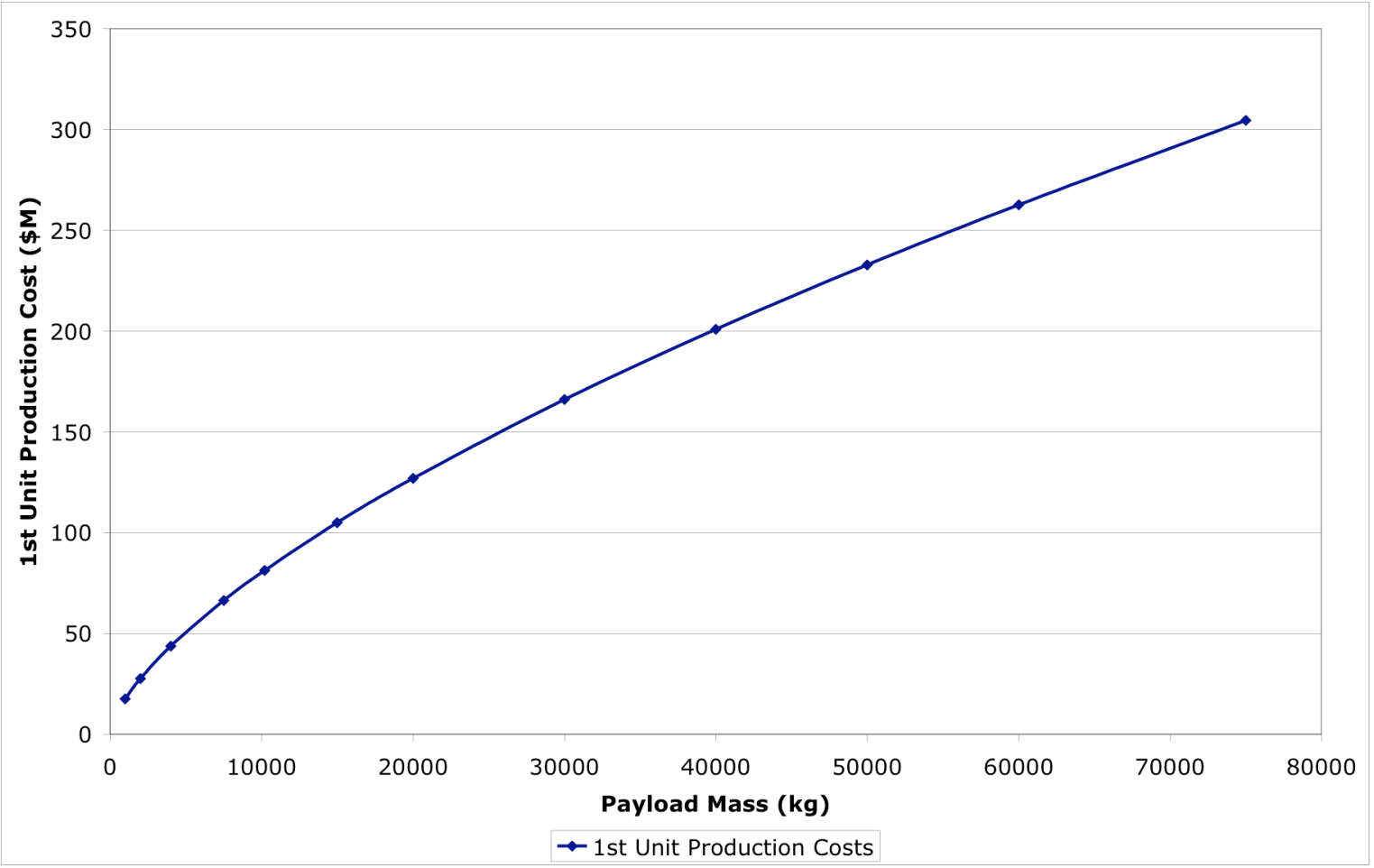

Figure 3.3: Baseline Vehicle $1^{\text {st }}$ Unit Costs 


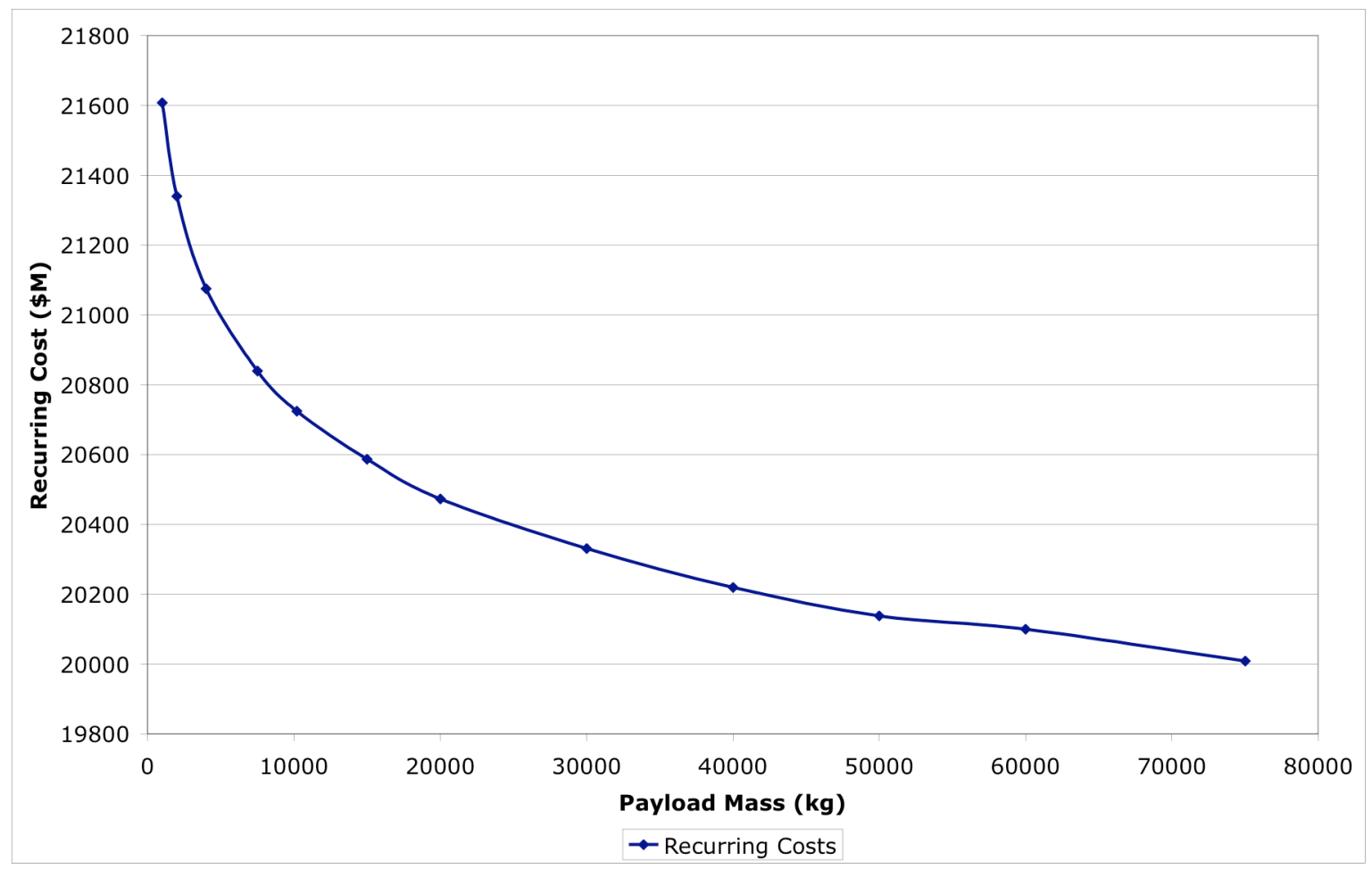

Figure 3.4: Baseline Vehicle Recurring Costs

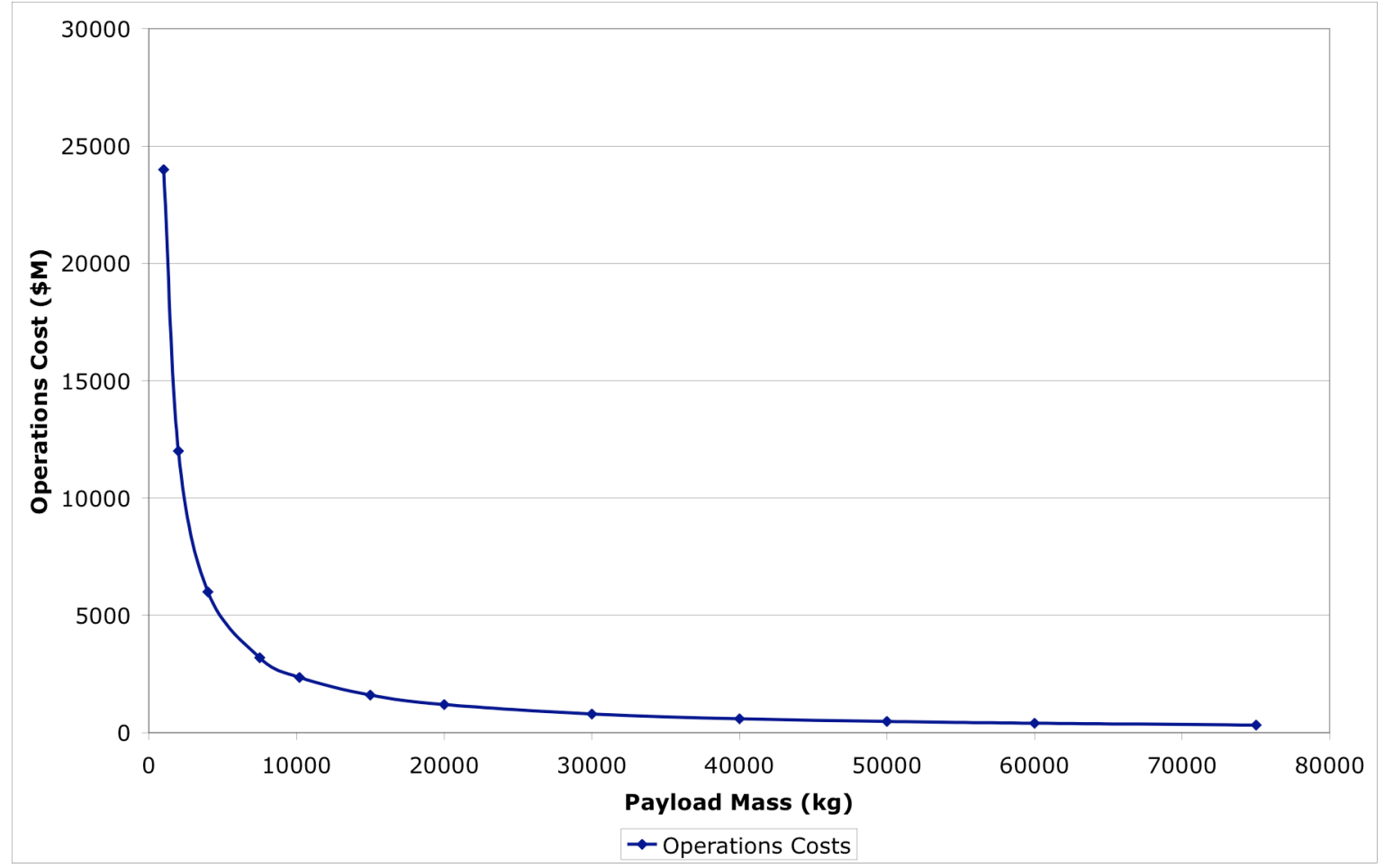

Figure 3.5: Baseline Vehicle Operations Costs 
The baseline launch vehicle reaches a cost minimum around a payload of 20,000 $\mathrm{kg}$ as shown in Figure 3.1. At lower and higher payloads the cost per kilogram payload to orbit rises. An examination of the subsequent component costs breakdowns can explain these trends. At small payloads the per flight operations costs dominates as shown in Figure 3.5. Because a substantial number of flights are required to get the total program payload mass to orbit at very low payloads, and the per flight operations cost is a function of the number of flights, the total payload costs are driven up. At large payloads the nonrecurring costs, shown in Figure 3.2 are dominant. With large payloads, there are fewer flights per vehicle required to lift the entire program payload mass into orbit. Therefore, there is less opportunity for the vehicle costs to be amortized over each flight during a vehicle's lifetime. First unit production costs in Figure 3.3 follow the same trend as the nonrecurring costs. This is because the first unit production costs are a function of inert mass. As the payload mass rises, more structural mass is needed to support it. More structural mass makes the production of the first unit more costly. The recurring costs, shown in Figure 3.4, decline at a small rate as the payload increases for the baseline expendable vehicle. At low payloads, the recurring costs are higher due to a greater number of vehicles produced than at high payloads. But the effects of the learning curve keep the recurring costs in check. In assembly line production, the more units built, the cheaper the $n^{\text {th }}$ unit will be when compared to the initial units. At low payloads, there are more vehicles required so the assembly process has more opportunity to learn and improve, thereby driving costs down. At higher payloads, fewer vehicles are built so there are less total production costs. 


\section{Variation of Parameters and Results}

The key independent variables inert mass fraction $(\delta)$ and refurbishment fraction

$\left(f_{\text {refurb }}\right)$ are manipulated within a range set by limitations of technology. As mentioned in Chapter 3.2 the historical range for $\delta$ is 0.04 to 0.20 for vehicles with rocket engines and 0.20 to 0.40 to stages with airbreathing engines. The range for $f_{\text {refurb }}$ is 0 for an entirely expendable vehicle to 0.20 for complex reusable vehicles with high refurbishment costs. A strong correlation between refurbishment fraction and number of flights per vehicle for reusable vehicles is found. For two stage vehicles the optimum change in velocity performed by each stage (staging $\Delta V$ ) is calculated for each scenario to minimize the payload cost.

\subsection{Inert mass fraction}

Since the SVLCM model estimates costs based on inert mass, it is intuitively obvious that as inert mass fraction increases, the payload cost to orbit rises; this trend is shown in Figure 4.1. 


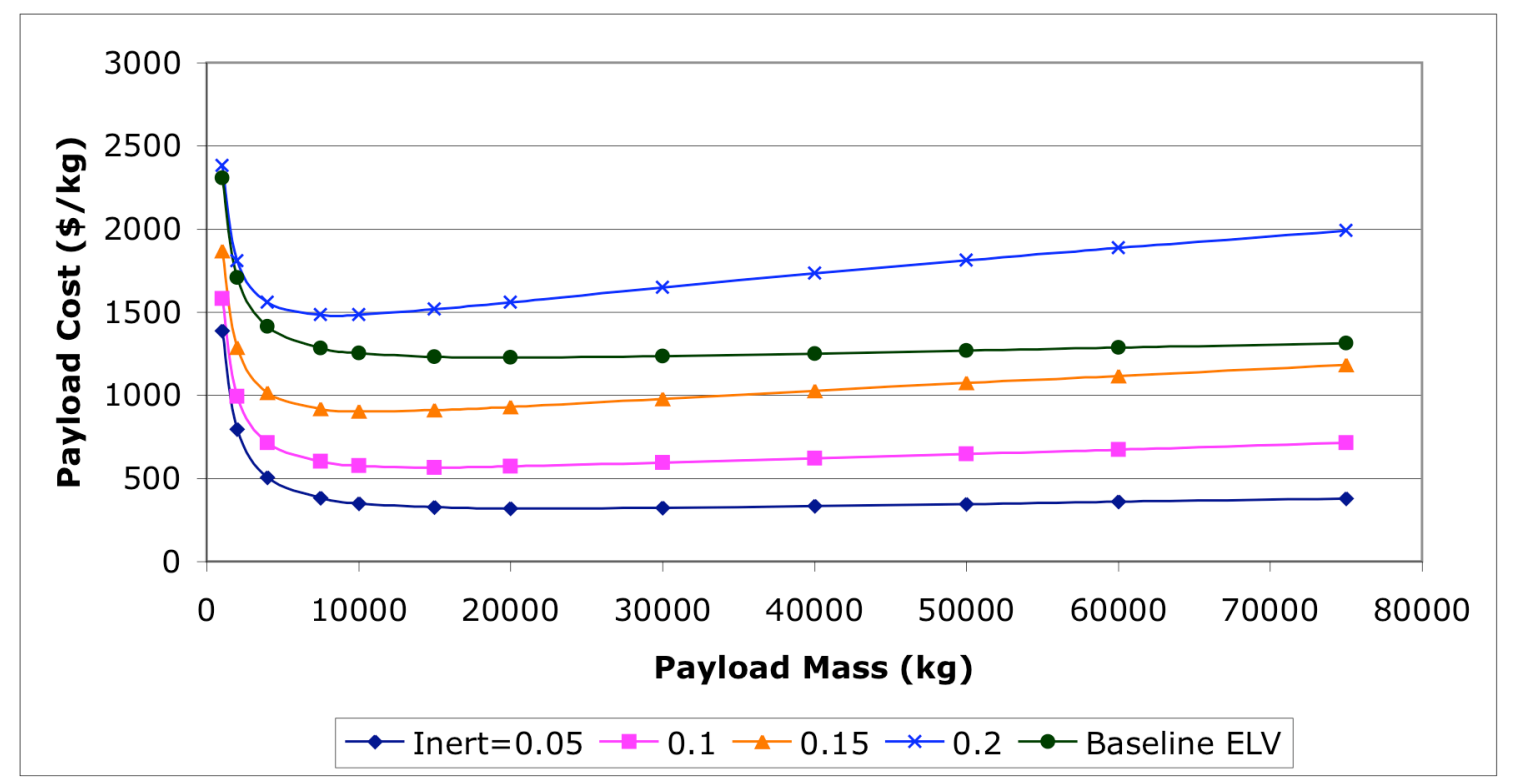

Figure 4.1: Inert mass fractions

It is interesting to note that, as the payload mass is varied from 0 to $75,000 \mathrm{~kg}$, the payload costs always exhibit a minimum at an intermediate value of payload mass. An examination of the component costs explain the trends exhibited: at very low payload masses, the per-flight operations cost dominates due to the large number of flights. At the high end of payload mass, the vehicles are large enough that costs are dominated by nonrecurring and refurbishment costs. With fewer flights per vehicle, there is less opportunity for the vehicle costs to be amortized over each flight during a vehicle's lifetime. The baseline ELV has a higher payload cost than all but vehicles at the highest end (0.2) of the inert mass fraction scale.

The specific value of optimum payload mass to minimize payload cost to orbit over the program occurs at a lower payload mass for each respective higher inert mass fraction. This is consistent with the observed trends, as higher inert mass fractions add to nonrecurring and refurbishment costs, and thus force the optimum payload size (and corresponding vehicle size) lower for minimum costs. The minimum for an inert mass 
fraction of 0.05 occurs closer to $20,000 \mathrm{~kg}$ while the minimum for an inert mass fraction of 0.20 occurs before $10,000 \mathrm{~kg}$. The minimum for the baseline expendable vehicle with a low inert mass fraction of 0.078 occurs around $20,000 \mathrm{~kg}$.

It is worth noting that all of these minimum cost payload sizes are subsumed within the range of current launch vehicles; in fact, even the highest inert mass fraction yields an optimum payload size less than that of the space shuttle or a heavy-lift EELV. While there may be operational considerations driving the current NASA desire for $100,000 \mathrm{~kg}$ payload launch vehicles for the Vision for Space Exploration, these Saturn V class launch vehicles are not likely to provide minimum launch costs.

\subsubsection{Vehicle inert mass economy of scale}

Having demonstrated the utility of inert mass fraction as a vehicle-level estimation parameter for categorizing program costs, the next logical step is to arrive at supportable estimates for $\delta$ as a function of launch vehicle design choices, such as type of propulsion system. Historical systems, such as classical multistage rocket system, can be analyzed by the use of known databases to find estimating relations for $\delta$. The results of this regression analysis, using historical data [15] from multiple stage vehicles including Delta, Soyuz, Saturn, Taurus, Pegasus and the Shuttle are shown in Figure 4.2. The historical vehicle stages are divided into four groups by propellant type (cryogenic, petroleum, solid and storable). 

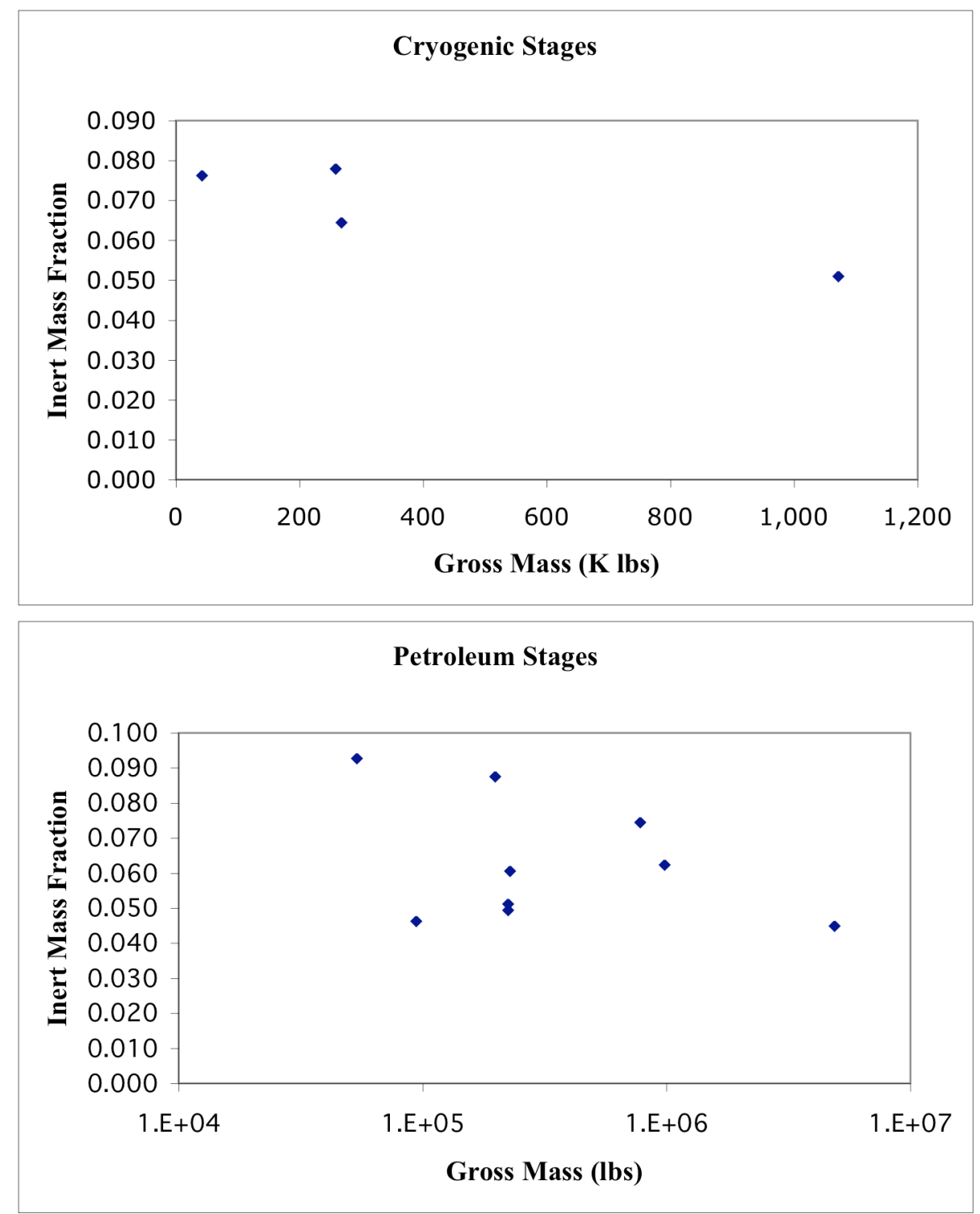

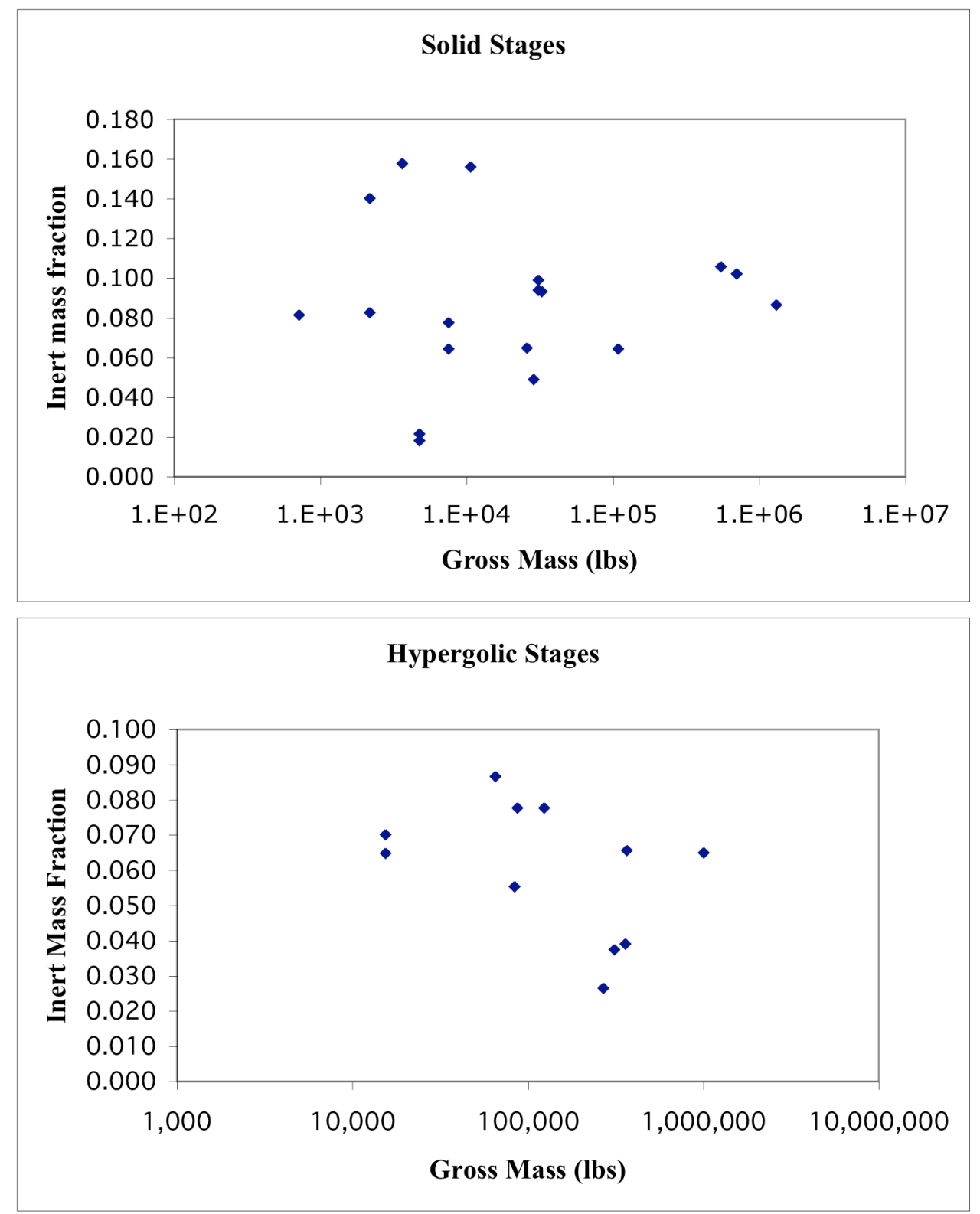

Figure 4.2: Gross mass vs. inert mass fraction

Multiple classical launch vehicle estimation algorithms [5,11] assume a physical economy of scale: increasing the absolute size of a stage corresponds to lower inert mass fractions, as increasing scale correlates to increasing structural efficiencies. By visual inspection, some aspects of this trend may be seen in the data of Figure 4.2. However, none of the data sets have mathematically acceptable regression trends, as substantial variations in $\delta$ across vehicle designs prevents an acceptable curve fit in three of the 
cases, and the fourth (cryogenic stages) has insufficient data points for a meaningful trend.

\subsubsection{Variable inert mass fraction}

Although there are no statistically significant trends to inert mass fraction as a function of vehicle gross lift-off mass, an intuitive examination of the regression analysis shows that there could be some reason to at least examine the effect of an economy of scale for vehicle inert mass. It may be valuable to do an analysis based on changing inert mass fraction as gross mass and propellant mass increases or decreases and how these variable inert mass fractions affect launch costs. Since gross mass is a function of payload mass, the effect of an economy of scale of gross mass on inert mass can be examined by changing inert mass fraction in increments as payload mass changes. Since reusable vehicles usually have more inert mass than a similarly sized expendable vehicle due to extra weight required for recovery systems, different ranges of inert mass fractions are applied for reusable and expendable launch vehicles.

The ELV variable and RLV variable inert mass fraction values follow Koelle's launch vehicle estimation algorithm [5] in that larger payloads requiring larger stages have lower inert mass fractions than their smaller counterparts due to increasing structural efficiencies. The values for variable inert mass fraction based on changing payload mass are approximated using a logarithmic fit. A logarithmic curve is a good fit because both curves fit well to Koelle's mass fraction curves from TRANSCOST. It should be noted that Koelle's definition of mass fraction varies from the definition of 
inert mass fraction presented here. Koelle's mass fraction is the dry mass plus residuals and gases at cutoff, without the engine mass, related to the propellant mass. Logarithmic curves of inert mass fraction changing depending on gross mass for the ELV and RLV are shown in Figure 4.3 below.

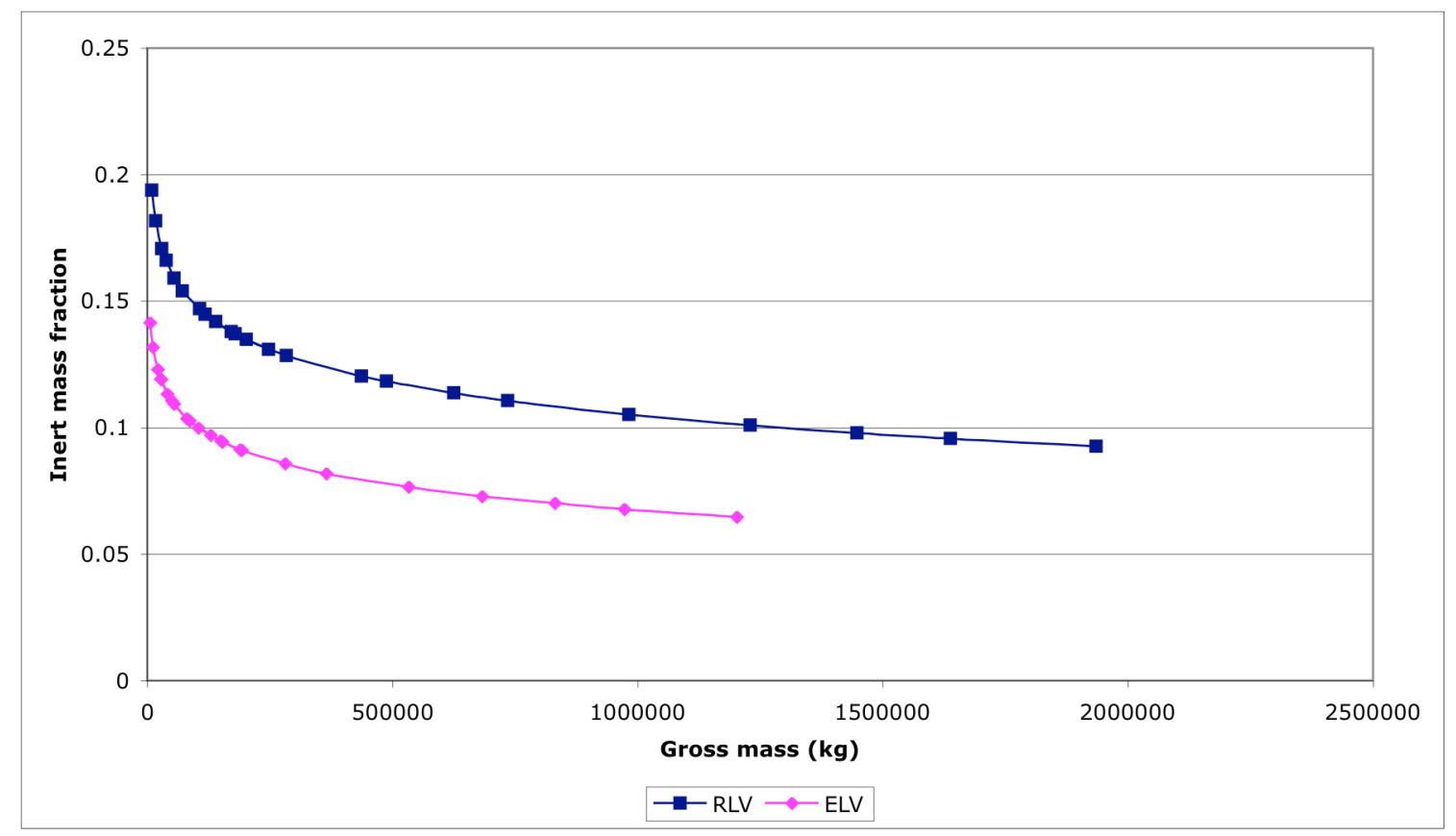

Figure 4.3: Logarithmic fit curves for variable inert mass fraction

If the launch vehicle is a multistage vehicle then the second stage is sized smaller, thereby assuming decreasing structural efficiencies. Therefore the highest inert mass fractions are seen at the second stage of vehicles lifting small payloads to orbit and the lowest inert mass fractions are seen at the first stage of vehicles lifting large payloads to orbit.

The effects of varying inert mass fraction on payload cost to orbit can be seen in Figure 4.4 below. 


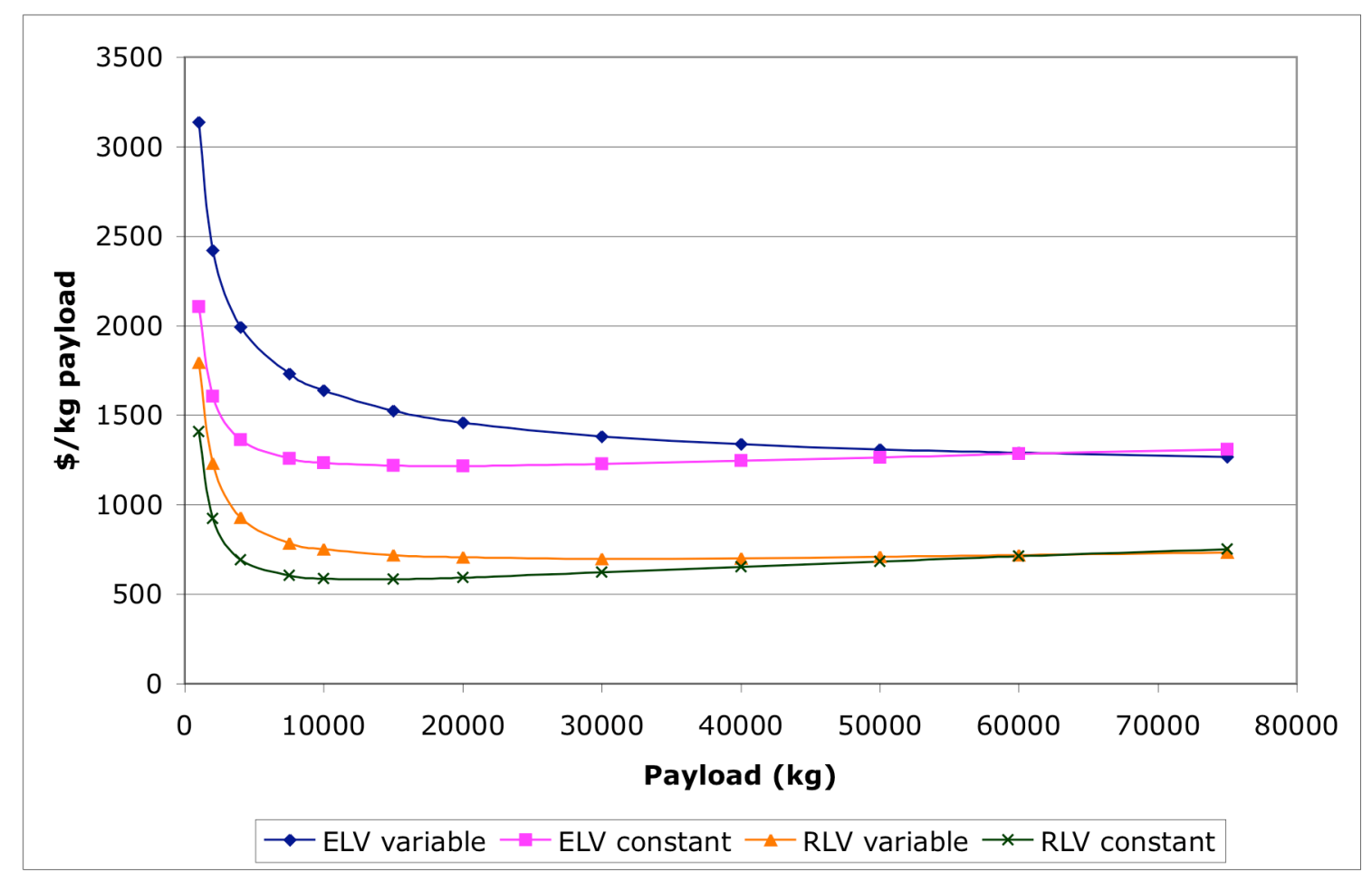

Figure 4.4: Payload cost to orbit for variable and constant inert mass fraction

These data compare the payload costs reusable and expendable launch vehicles with the constant and varying inert mass fraction trends shown in Figure 4.3. The results shown are intuitive for the most part. The payload costs of the ELV and RLV with constant inert mass fractions are lower than their higher variable inert mass fractions counterparts. The vehicles with variable inert mass fractions cost more than the vehicles with constant inert mass fractions at lower payloads because the variable inert mass fractions are higher at lower payloads. For the constant inert mass fractions the minimum costs occur at payloads near $20,000 \mathrm{~kg}$. The payload at which a cost minimum occurs has shifted to higher payload values, above $60,000 \mathrm{~kg}$, for both the ELV and RLV variable inert mass fraction cases. Variable inert mass fractions assume lower values for larger vehicles. Since the CERs are based on inert mass of a vehicle, the costs for large vehicles will decrease with variable inert mass fractions. 


\subsubsection{Airbreathing first stages}

Examining vehicles, both theoretical and real, with some type of airbreathing engine, performs a second approach to inert mass fraction estimation. The purpose of this study is to assign an estimated range of values for inert mass fractions of stages with airbreathing engines. Those results can be found in Table 4.1 below, along with some rocket-based parameters taken from the regression analysis above.

\begin{tabular}{|l|l|}
\hline Vehicle/Stage & Inert mass fraction \\
\hline Space Shuttle & 0.113 \\
\hline SSTO Rocket & 0.081 \\
\hline SSTO Airbreather/Rocket & 0.261 \\
\hline SSTO HTHL Airbreather/Rocket & 0.261 \\
\hline SSTO RBCC & 0.178 \\
\hline TSTO Airbreather/Rocket & 0.379 \\
\hline TSTO HTHL S1-RBCC & 0.232 \\
S2-Rocket & 0.176 \\
\hline TSTO TBCC Stage 1-Launcher & $0.379-0.425$ \\
\hline TSTO Spaceplane Stage 2-Orbiter & $0.318-0.414$ \\
STTO TBCC Stage 1 & $0.127-0.173$ \\
Stage 2 & 0.371 \\
Stage 2 & 0.198 \\
\hline TSTO ACES Stage 1 & 0.413 \\
\hline
\end{tabular}

Table 4.1: Inert mass fractions of air breathing vehicles

Based on these figures an inert mass fraction in the area of 0.35 is a safe assumption for vehicle stages with airbreathing engines. This figure will be slightly higher for turbine based combined cycle (TBCC) engines and slightly lower for rocket based combined cycle (RBCC) engines. For this study, the fuel-specific impulse of air breathing stages is set at 2000 seconds. This is at the low end of an acceptable range of specific impulse for a jet engine (2000-3000 seconds), accounting for decreased efficiency of an airbreathing engine operating at high speeds and altitudes. 


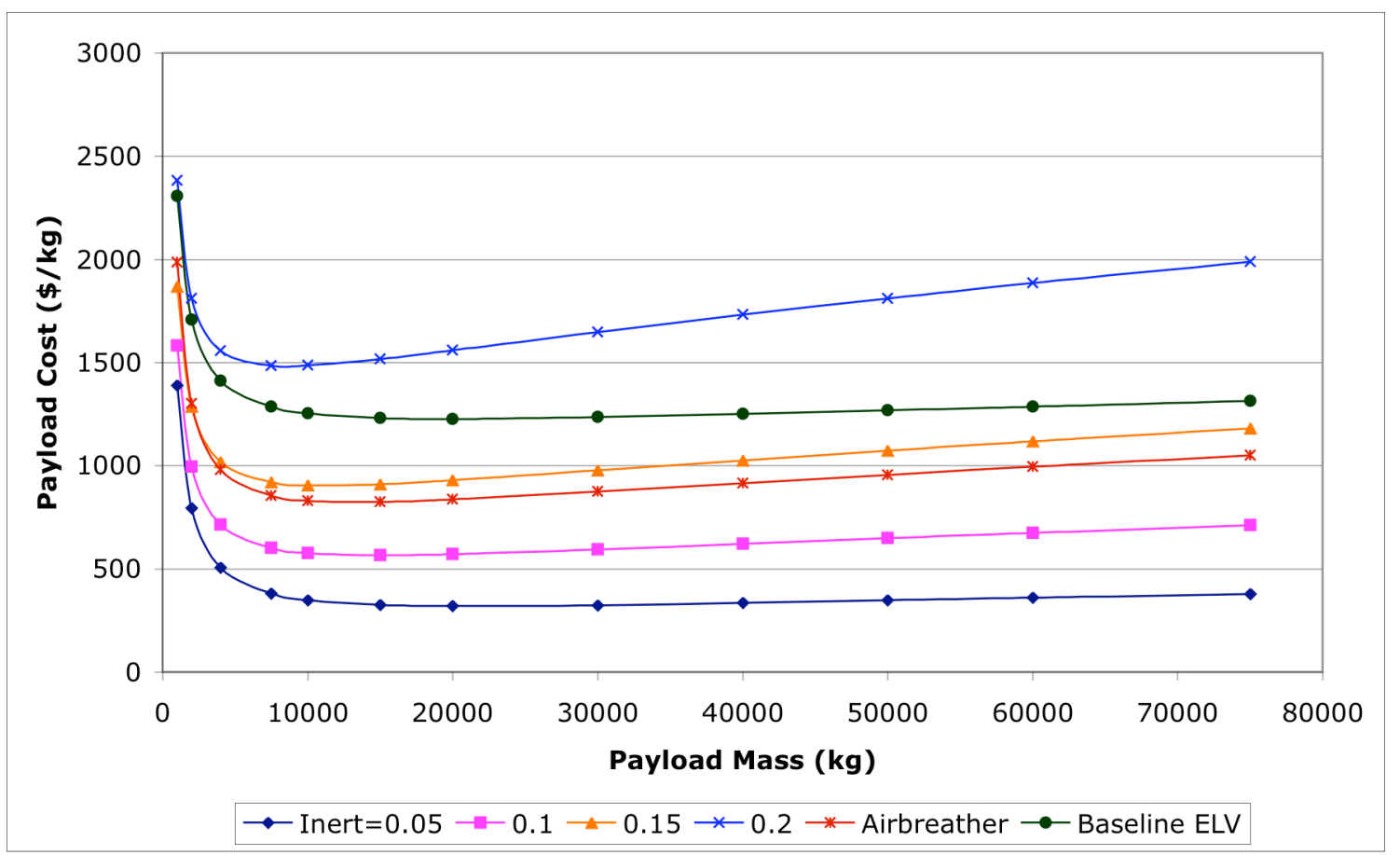

Figure 4.5: Inert mass fractions with air breather

Figure 4.5 is similar to Figure 4.1, but the latter now includes a set of data representing a vehicle with an air breathing first stage. This data is labeled "air breather" on the graph and is calculated using the assumptions for an air breathing stage stated above. The airbreather is more costly than rocket based vehicles with inert mass fractions under 0.15 ; however, it remains less costly than rocket based vehicles with inert mass fractions of 0.2 and above. The airbreather is also less costly than baseline ELV. The airbreather modeled here has a larger inert mass fraction $(0.35)$ but the efficiency of the engine (fuel-specific impulse $=2000$ seconds) keeps the overall vehicle size down, which in turn limits the vehicle cost elements and keeps the payload cost down. Airbreathing systems are far more complex than rocket systems. There isn't sufficient data available from a hybrid system with an airbreathing first stage and a rocket upper stage to accurately model how additional complexities will affect vehicle costs. It is 
likely that development costs will reach higher than even the high inert mass fraction considered here can account for. Also operations complexity factors must be considered to drive up the cost of an airbreathing first stage.

For TSTO vehicles with airbreathing first stages, the $2^{\text {nd }}$ stage and overall vehicle will be smaller the higher the flight velocity at which staging occurs. Staging Mach numbers ranging from 5-14 are a good range for airbreathing systems, encompassing ramjet and scramjet engines. $[16,17]$ In a hybrid vehicle where the $1^{\text {st }}$ stage transitions from a ramjet or scramjet to rocket propulsion, the first stage uses less propellant than a standard rocket based stage because the high Isp airbreathing systems is operated over a large portion of the flight within the atmosphere. As the velocity at which staging occurs increases, the distance traveled requirement for the rocket based propulsion system in the $2^{\text {nd }}$ stage decreases, thereby decreasing the mass of chemical propellant needed and the overall mass of the vehicle. Also the payload cost of the vehicle goes down since less propellant is needed less structural mass is needed to support the propellant. These trends are shown in Figure 4.6. 


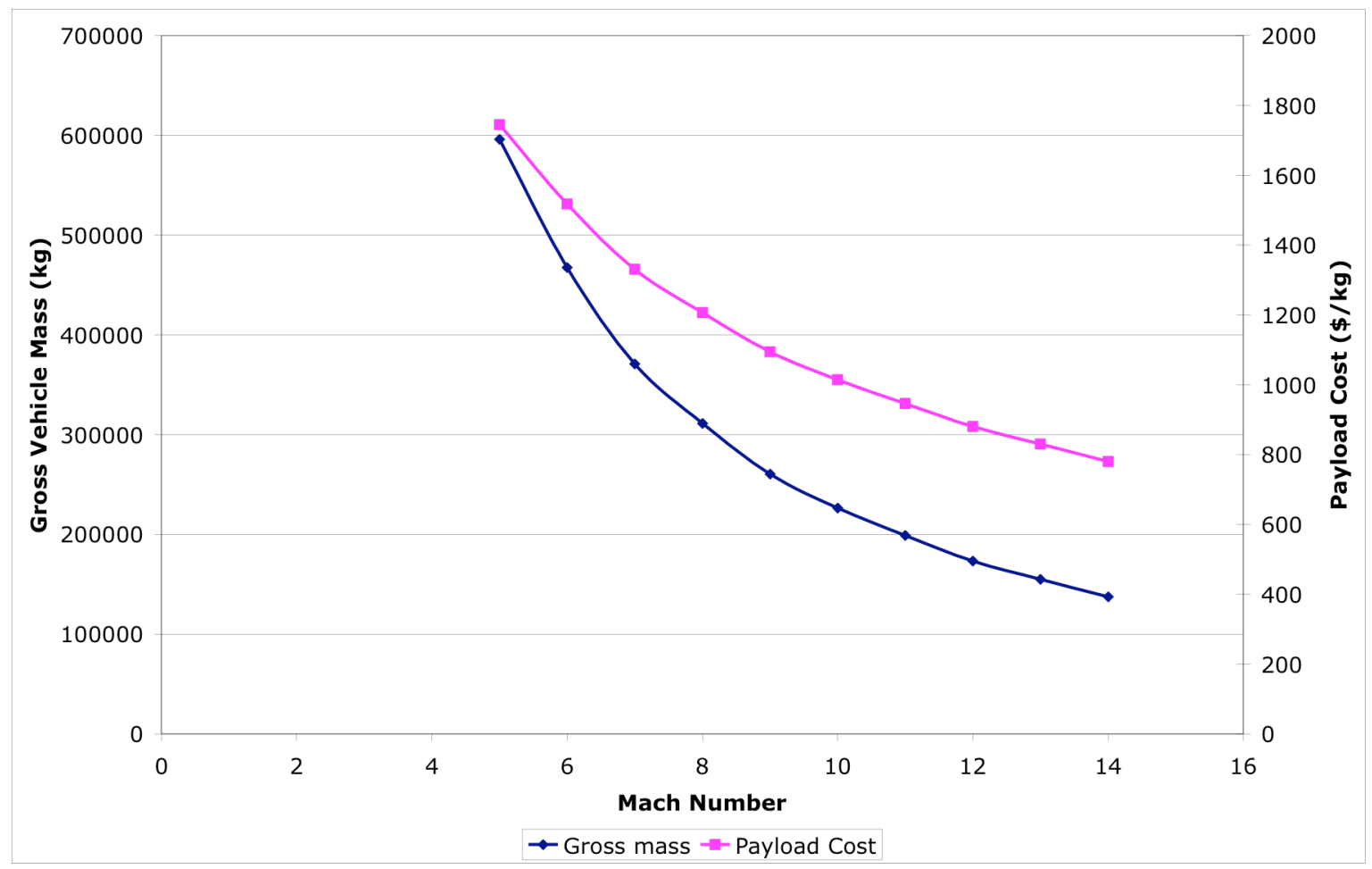

Figure 4.6: Airbreathing Staging Mach 5-14

Carried to the extreme a minimum payload cost is reached if all of the propellant in stage 2 is eliminated and all the change in velocity occurs in stage 1 . In the real world an airbreathing engine only has a certain operating envelope and above a certain Mach number and altitude range the rocket engine in the $2^{\text {nd }}$ stage must be used.

\subsection{Refurbishment fraction and number of flights per vehicle}

Refurbishment costs are those costs associated with maintenance and repair on reusable vehicles between flights. The refurbishment fraction is defined as the percentage of the first unit production cost that is required for average post-flight refurbishment of a reusable launch vehicle for subsequent launches. A range of refurbishment fractions 
from $1-20 \%$ was considered. The current space shuttle orbiter generally falls into the 10$20 \%$ range for refurbishment fraction.

As can be seen from Figure 4.7, increasing the refurbishment fraction of a vehicle increases the payload cost at any given payload mass. Even a high refurbishment fraction, such as 0.2 , is still more cost effective than an identical expendable vehicle,

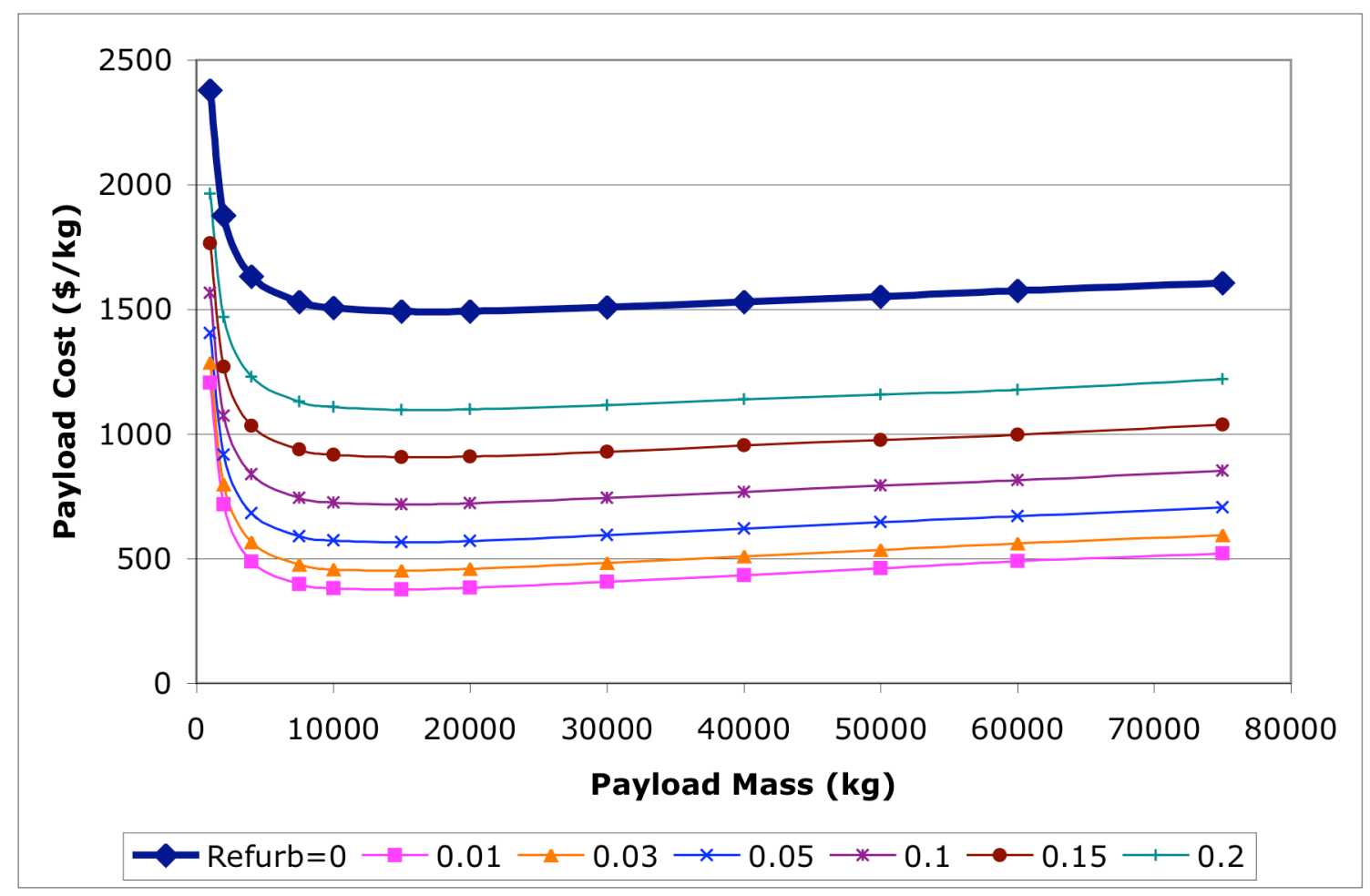

Figure 4.7: Refurbishment Fraction

represented by setting refurbishment fraction to 0 and number of flights per vehicle to 1 . The refurbishment fraction would have to be increased to the neighborhood of 0.4-0.45 for a reusable vehicle to become less cost effective than an expendable vehicle. This is due to the high production costs associated with expendable vehicles. The production costs of reusable vehicles are amortized over their lifetime due to multiple flights per vehicle. It should be pointed out that the specific comparison here is between identical 
reusable and expendable vehicles: this analysis does not take into account the fact that an expendable vehicle is inevitably lighter in weight due to the lack of need for carrying recovery systems, nor that reusable vehicles typically require more advanced technologies for viability, thus requiring a cost premium for both nonrecurring and recurring costs at the same physical size as a corresponding expendable vehicle. Taking into account the mass and cost premiums for a reusable vehicle over an expendable through a modest increase of the inert mass fraction has an immediate effect on the sensitivity of the cost efficiency of a reusable vs. an expendable. For example an expendable is assumed to have an inert mass fraction of 0.078 and the inert mass fraction is raised to 0.12 to simulate the need for carrying recovery systems and developing more advanced technology, thereby increasing the nonrecurring costs of a similar reusable vehicle carrying the same payload size. At this point if the refurbishment fraction is raised to the high end of the range of values considered in Figure $4.7(0.17-0.20)$ the expendable vehicle is actually more cost effective than the reusable as seen in Figure 4.8. 


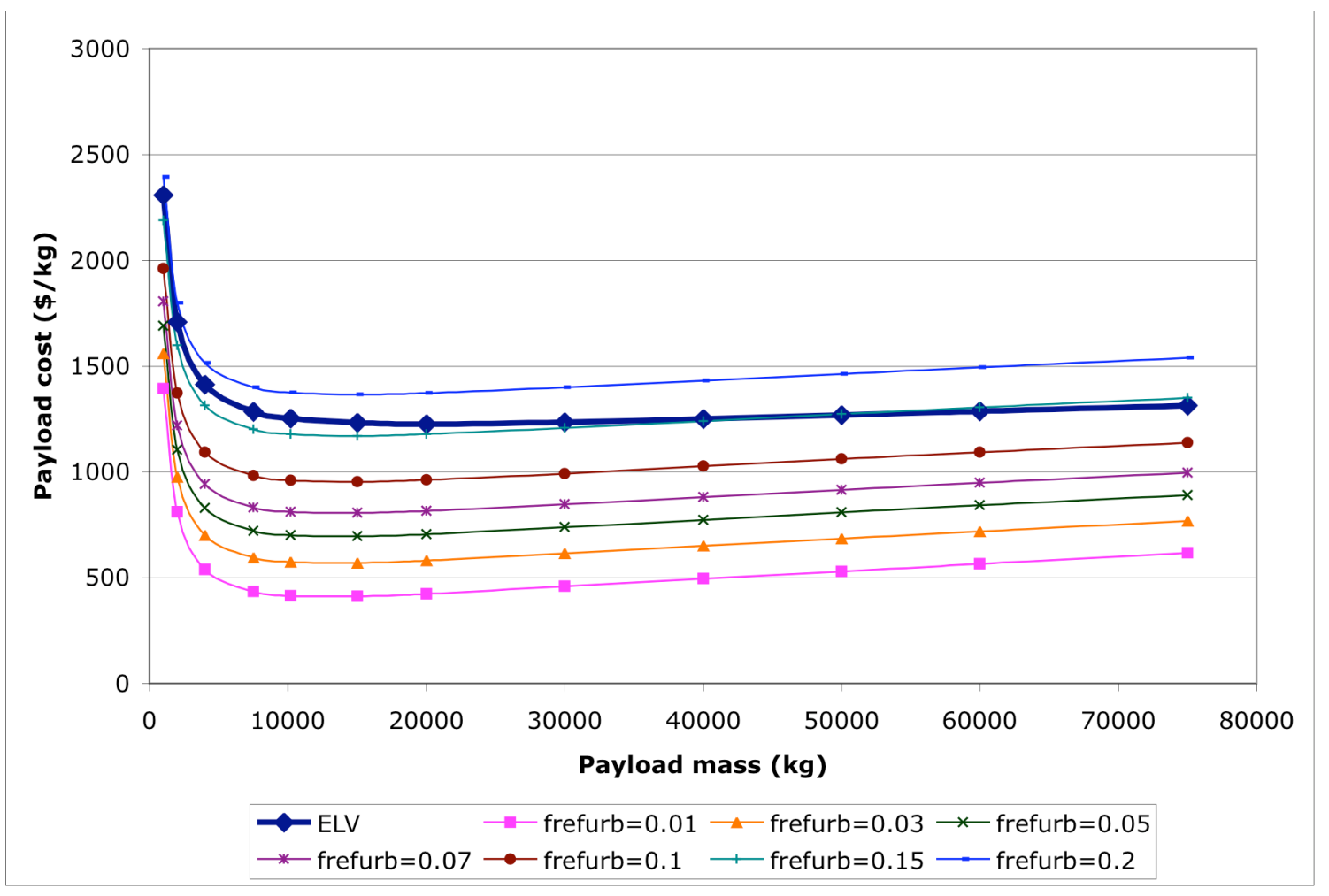

Figure 4.8: Refurbishment fraction corrected for RLV vs. ELV

For this case the cost of recovery and refurbishment of a reusable with additional dry mass is more than the cost of building a new unit of a similar expendable.

Figures 4.7 and 4.8 show an interesting result with regards to refurbishment fraction and payload mass. The optimum payload size for minimum costs occurs between 10,000-20,000 $\mathrm{kg}$ for all refurbishment fractions in the range considered, from $0-0.20$. The optimum payload size of a vehicle is independent of the level of reusability. An interesting complement to the refurbishment fraction is the number of flights flown per vehicle. While it might intuitively seem that one would always want to fly a reusable vehicle as many times as possible, analysis indicates that, beyond a certain point, the reusable vehicle fleet size becomes so small that almost no advantageous effects of the learning curve are achieved. Taken to the extreme, if one vehicle could fly every 
mission in the program, it would be a "hand-built" vehicle, with corresponding high costs for refurbishment parts.

To better understand the effects of number of flights per vehicle on the payload launch costs, sensitivity analysis was run to optimize the optimum number of flights per vehicle for each of a range of refurbishment fractions. First a baseline case of a reusable TSTO vehicle with $\delta_{I}=\delta_{2}=0.12$ and $I_{s p 1}=I_{s p 2}=450$ seconds was established. The results are shown in Table 4.2 below.

\begin{tabular}{|c|c|c|c|c|}
\hline$f_{\text {refurb }}$ & Optimum $\boldsymbol{N}_{f p \boldsymbol{v}}$ & $\begin{array}{c}\text { Optimum Payload } \\
\text { Size }\end{array}$ & $\begin{array}{c}\text { Optimum } \boldsymbol{d} \boldsymbol{v} \\
\text { Split }\end{array}$ & $\begin{array}{c}\text { \$/kg at Optimum } \\
\text { Payload }\end{array}$ \\
\hline 0.01 & 215 & 13700 & 0.625 & 314 \\
\hline 0.03 & 72 & 14000 & 0.622 & 432 \\
\hline 0.05 & 43 & 14200 & 0.621 & 524 \\
\hline 0.07 & 31 & 14200 & 0.620 & 605 \\
\hline 0.1 & 21 & 14400 & 0.619 & 714 \\
\hline 0.15 & 14 & 15000 & 0.619 & 874 \\
\hline 0.2 & 11 & 15400 & 0.618 & 1017 \\
\hline
\end{tabular}

Table 4.2: Refurbishment fraction and flights per vehicle at $\delta=0.12$ and $I_{s p}=450$

This analysis shows that low refurbishment fractions (with correspondingly low refurbishment costs) optimize to a large number of flights per vehicle. Given the relatively low refurbishment costs, there is a clear benefit to a long vehicle lifetime as it allows the amortization of nonrecurring costs over a large number of flights. As the refurbishment fraction moves into the shuttle range $(0.1 \sim 0.2)$, the optimum number of flights per vehicle drops precipitously, into the range of only 10-20 flights per vehicle. There is a greater benefit to increasing the production run (with resultant learning curve efficiencies) than extending the life of a small expensive fleet for a vehicle with high refurbishment costs. If a vehicle has refurbishment costs of $20 \%$, flying it 5 times is 
equivalent to buying a new vehicle. This is what's happening with the Space Shuttle now. The Shuttle is a small fleet of vehicles, for which learning curve effects are minimal, with high refurbishment costs. Also there aren't enough spare parts for the vehicles currently in use, so this further detracts from possible learning curve effects. Each new spare part is essentially a custom built part since production lines are shut down. The alternative to this is switching out parts between vehicles, which is inefficient. In all cases, refurbishment costs are a substantial portion of the overall payload launch costs, as reducing refurbishment rates dramatically reduces overall payload costs. The other significant trend from Table 4.2 is that there is very little difference across the cases in absolute optimum payload mass or in the velocity increment at staging conditions. While these parameters are still included in all subsequent analyses, the values for these second-order variable results will not be further documented in this paper; instead, we will focus on trends in cost per kilogram of payload, along with number of flights per vehicle to produce the minimum payload charges. Using refurbishment fraction as the independent variable, the trends relating optimum number of flights per vehicle with payload costs are shown in Figure 4.9. 


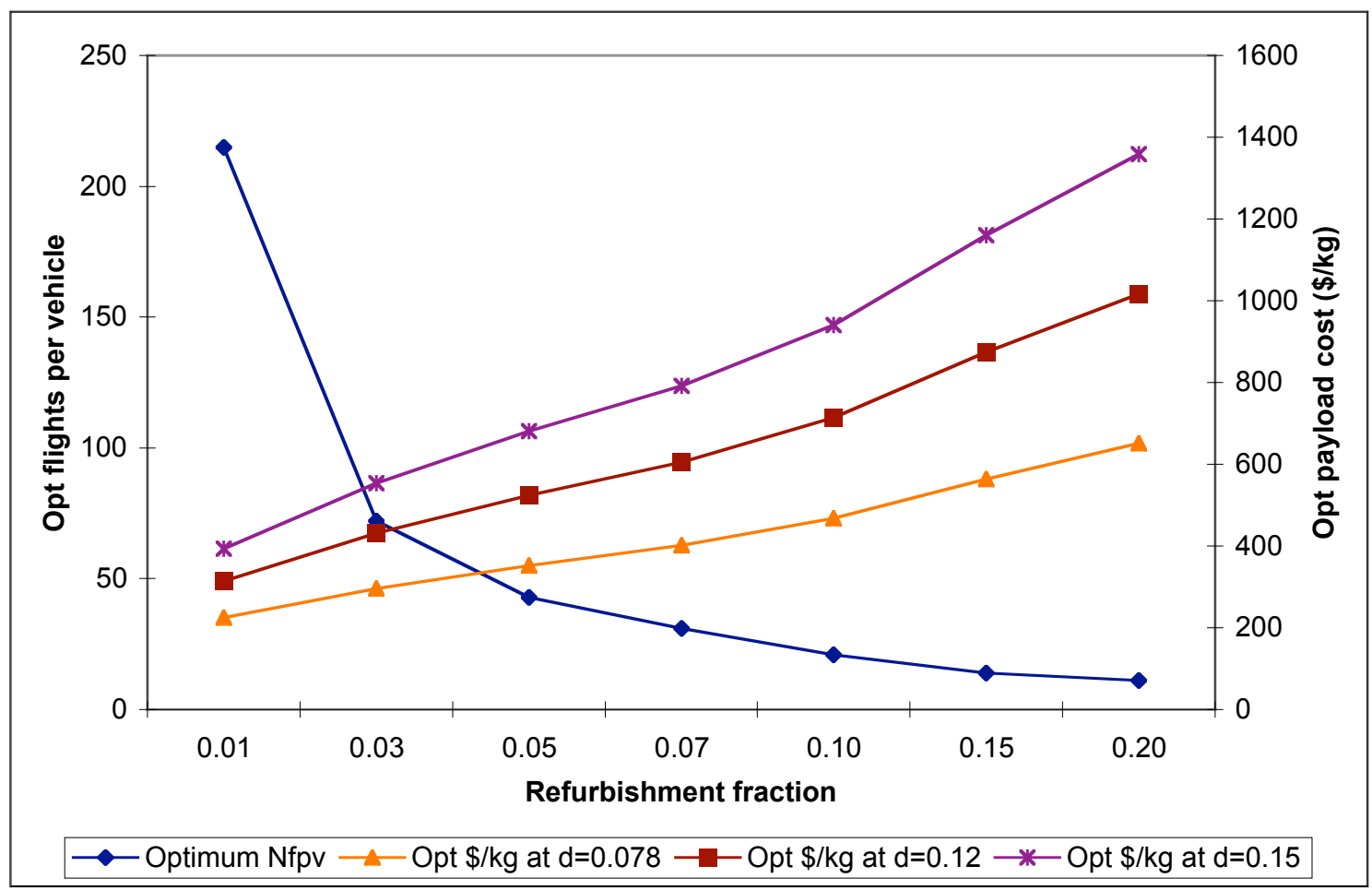

Figure 4.9: Refurbishment fractions and flights per vehicle at $\delta=0.078,0.12,0.15$ and $I_{s p}=450 \mathrm{sec}$

To determine the effect of changing inert mass fraction on the relationship between refurbishment fraction, $N_{f p v}$, payload size, payload cost and dv split, a sensitivity analysis was performed by raising and lowering the values for $\delta$ by a set factor. From the baseline value of $0.12, \delta$ was lowered to $\delta_{I}=\delta_{2}=0.078$, and then raised to 0.15 ; throughout these trials, the specific impulse was maintained at $I_{s p 1}=I_{s p 2}=450$ seconds.

Similarly, to determine the effect of changing $I_{s p}$ on the relationship between refurbishment fraction, $N_{f p v}$, payload size, payload cost and $d v$ split, the specific impulse values were set altered from the baseline $\mathrm{I}_{\mathrm{sp}}$ to $I_{s p 1}=I_{s p 2}=320$ seconds while holding $\delta_{I}=\delta_{2}=0.12$. The results are shown below in Figure 4.10. 


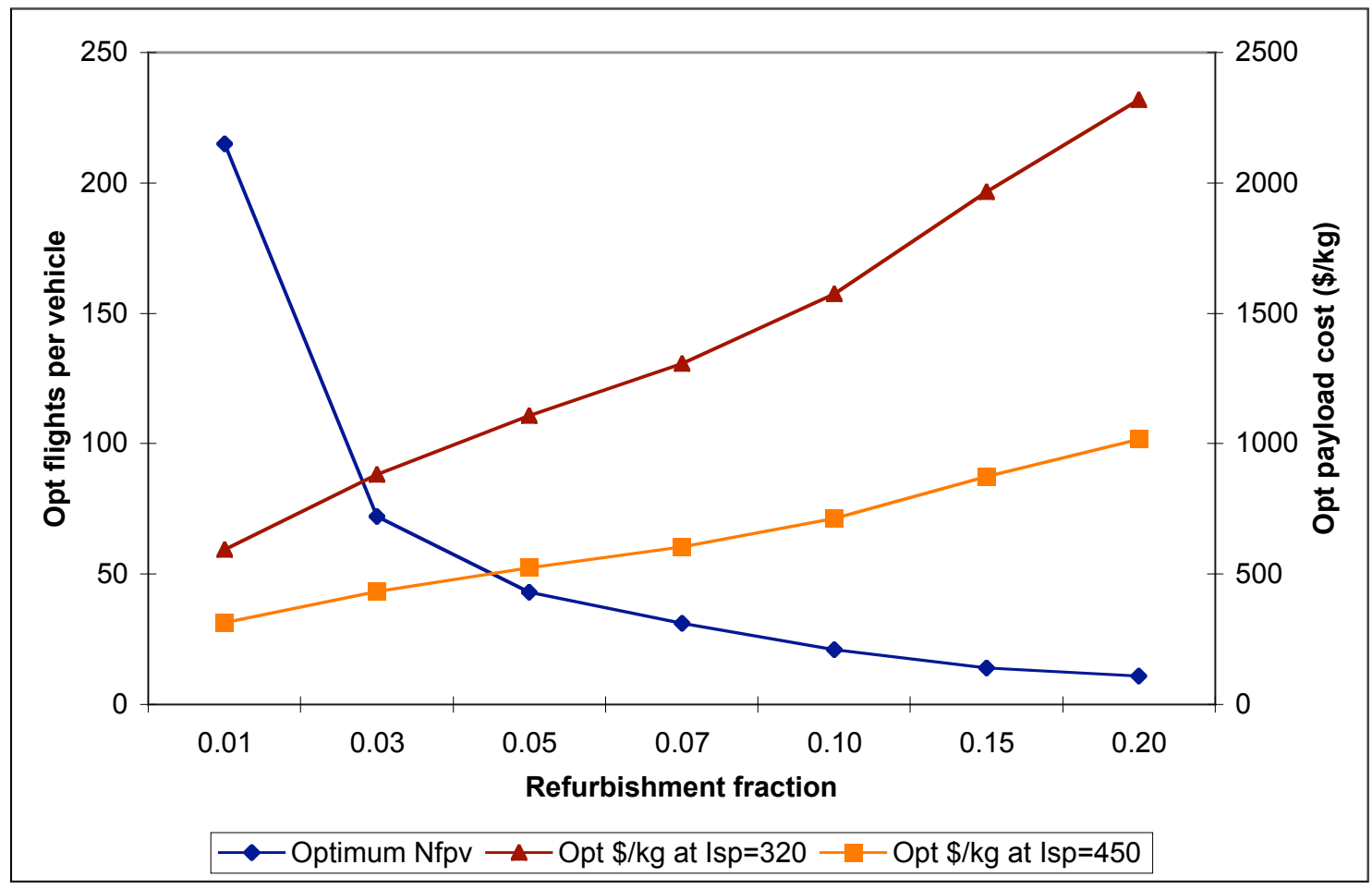

Figure 4.10: Refurbishment fraction and flights per vehicle at $\delta=0.12$ and $I_{s p}=320$, $450 \mathrm{sec}$

These sensitivity analyses show that changing $\delta$ and $I_{s p}$ does not have any effect on the optimum number of flights per vehicle for a given refurbishment fraction. Also, the optimum payload size stays nearly constant throughout, with only a few hundred kilograms variation across the various analyses. However, the payload costs per kilogram change dramatically, which illustrates the critical importance of refurbishment fraction on launch costs.

As mentioned earlier, there is a direct correlation between the refurbishment fraction and the optimum number of flights per vehicle. The optimum number of flights per vehicle, at a given refurbishment fraction, remains constant as the inert mass fraction, specific impulse or $\Delta \mathrm{V}$ split change.

The optimum number of flights per vehicle decreases as refurbishment fraction increases, due to the learning curve impact of extremely small fleet sizes and 
corresponding production runs. Since refurbishment fraction is a function of first unit production costs, refurbishment fractions as a cost driver are scaled with the overall size of the vehicle. Vehicles with a high refurbishment fraction have a higher payload cost than vehicles with a low refurbishment fraction. At currently demonstrated refurbishment rates (10-20\%), vehicle maintenance actions following each flight are a significant fraction of the costs to build the first vehicle. Unlike increasing the size of the production run, however, refurbishment costs (as evidenced by both the X-15 and Space Shuttle programs) evidence much lower rates of learning effects than vehicle production. Therefore, to reduce costs for a vehicle with a high refurbishment fraction, it is advantageous to reduce the number of flights flown by each vehicle to increase the size of the fleet production run, which in turn reduces both the cost per vehicle and the cost of necessary spares for the refurbishment process. One corollary of this observation is that as the payload size increases, the optimum number of flights per vehicle decreases. Higher vehicle production and flight rates for the program as a whole (as opposed to any single vehicle) still are the most important factor in minimizing payload launch costs. Also reusable vehicles that are expensive to refurbish (over $15 \%$ of the first unit cost) must be carefully examined to determine if it is actually cheaper to reuse the vehicle or produce a new expendable one. 


\section{Advanced Cost Analysis}

\subsection{Modularity}

The current study has shown the critical effects of learning curves, which are the driving effect towards smaller vehicle sizes in order to increase production and flight rates. One strategy to increase production run sizes is to adopt modular vehicle practices, as in the three identical common booster core modules of the Delta IV Heavy EELV. Rather than a single monolithic first stage, three identical modules reduce the total unique inert mass in the first stage (thus decreasing both nonrecurring and first unit production costs), as well as tripling the size of the first stage production run for a given number of launch vehicles produced. Other concepts in modular launch vehicles, such as the OTRAG [18] vehicle concepts from the 1970's, dramatically reduced the unique design mass by adopting designs with large numbers of identical modules. This approach should be better modeled and compared directly with more conventional designs, both reusable and expendable.

\subsubsection{Modularity methodology}

To apply modularity to this cost model, the $1^{\text {st }}$ stage inert mass is split up into identical modules. With a one module first stage representing the monolithic first stages modeled up until this point, vehicles with 1, 3, 5, and 7 modules are analyzed. A new parameter $N_{\text {units }}$ is defined as:

$$
N_{\text {units }}=N_{\text {veh }} * N_{\bmod s / v e h}
$$

$N_{v e h}$ is the number of vehicles required in the program given a total program payload mass and a payload mass per vehicle. $N_{\text {mods } / v e h}$ is set at $1,3,5$ or 7 modules in the first stage. 
To determine production costs and learning curve effects, each module is referenced as one unit. Therefore the term $M_{\text {gross }}$, which previously reflected the vehicle stage one gross mass, now is used to identify the gross mass of one module within stage one by dividing the vehicle gross mass by the number of modules in stage one. To reflect the costing changes associated with dividing stage one into modules, the term $N_{v e h}$ is replaced with $N_{\text {units }}$ in the appropriate equations. The recurring costs and refurbishment costs are affected as shown below:

$$
\begin{aligned}
& C_{r}=C_{1^{t}} * \frac{N_{\text {units }}^{\left(1+p_{\text {exp }}\right)}}{1+p_{\text {exp }}} \\
& C_{\text {refurb }}=\frac{C_{r}}{N_{\text {units }}} * f_{\text {refurb }} * N_{f t s} * N_{\text {units }}
\end{aligned}
$$

In the expression for refurbishment costs, the recurring cost is divided by the number of units in this case. However the number of flights must also be multiplied by the number of units since a flight contains more than one unit when the number of modules per vehicle is greater than one. Simplifying, the refurbishment costs expression turns out to be the same as introduced in Chapter 3.

$$
C_{\text {refurb }}=\frac{C_{r}}{N_{\text {veh }}} * f_{\text {refurb }} * N_{f t s}
$$

It should be noted that the values for refurbishment costs still change as the number of units change, since the refurbishment costs expression contains the recurring costs term, which is a function of the number of units.

The effects of implementing modules on the nonrecurring, recurring, refurbishment and overall payload costs for the vehicle first stage are illustrated below in Figures 5.1-5.4. The refurbishment fraction here is 0.05 . While the values of the costs 
will change for different refurbishment fractions, the visual trends will be similar in all cases of a reusable first stage.

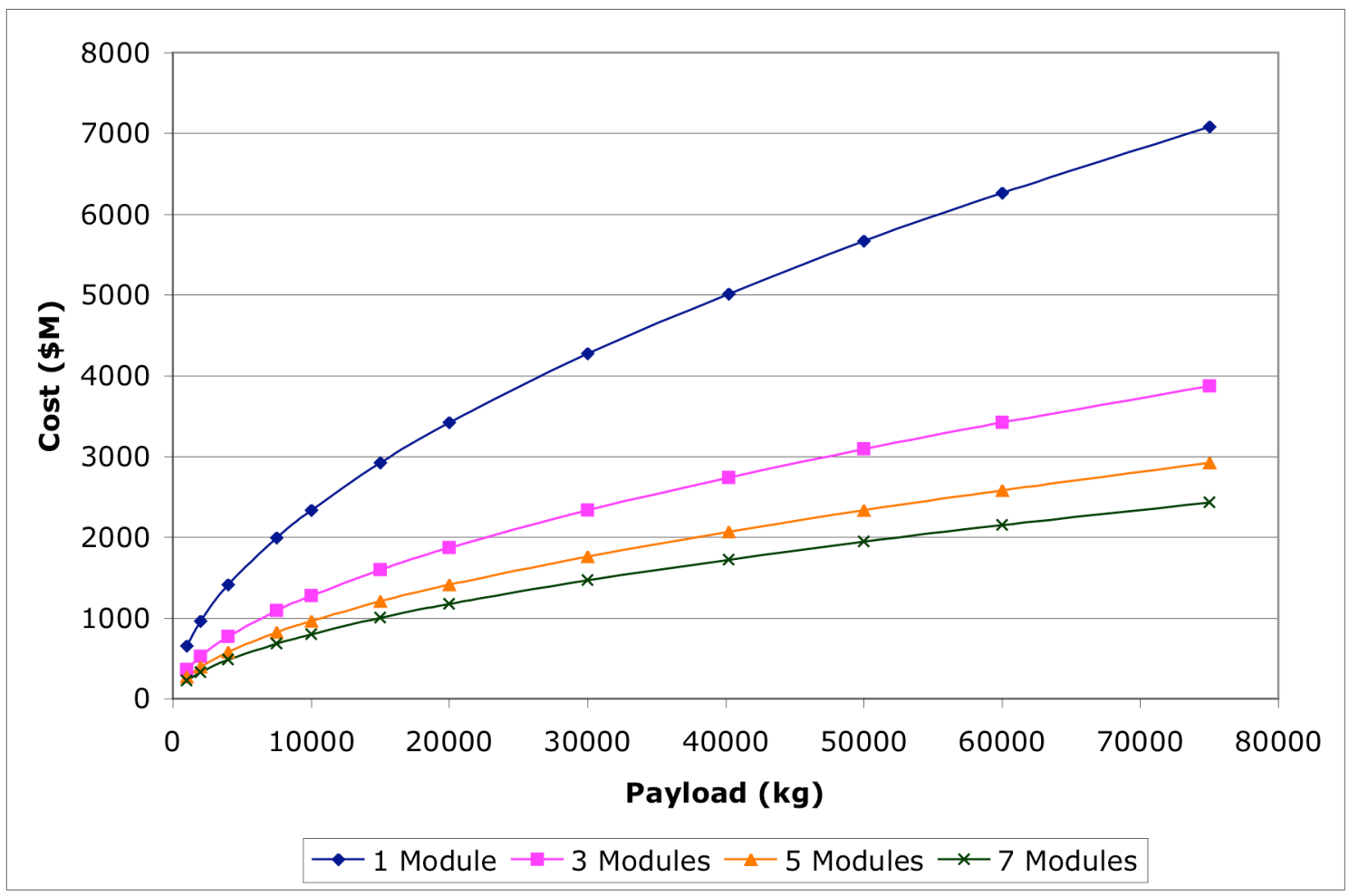

Figure 5.1: Nonrecurring costs $1^{\text {st }}$ stage

The nonrecurring costs decline as the number of modules in the first stage increases.

This is due to the assumption that all modules produced are identical and have the same inert mass, which is a fraction of the total stage inert mass. Due to the nonlinear relation used to represent these development costs, as the inert mass for each module goes down (as the number of modules goes up), the rate at which the development costs increase given an incremental increase in payload goes down. This is a simplified assumption however. With increasing the number of modules in the first stage, it is expected that there will be inert mass penalties and operations complexities resulting from the inherent increased difficulty in assembling multiple modules. These additional factors are examined later in this section. 


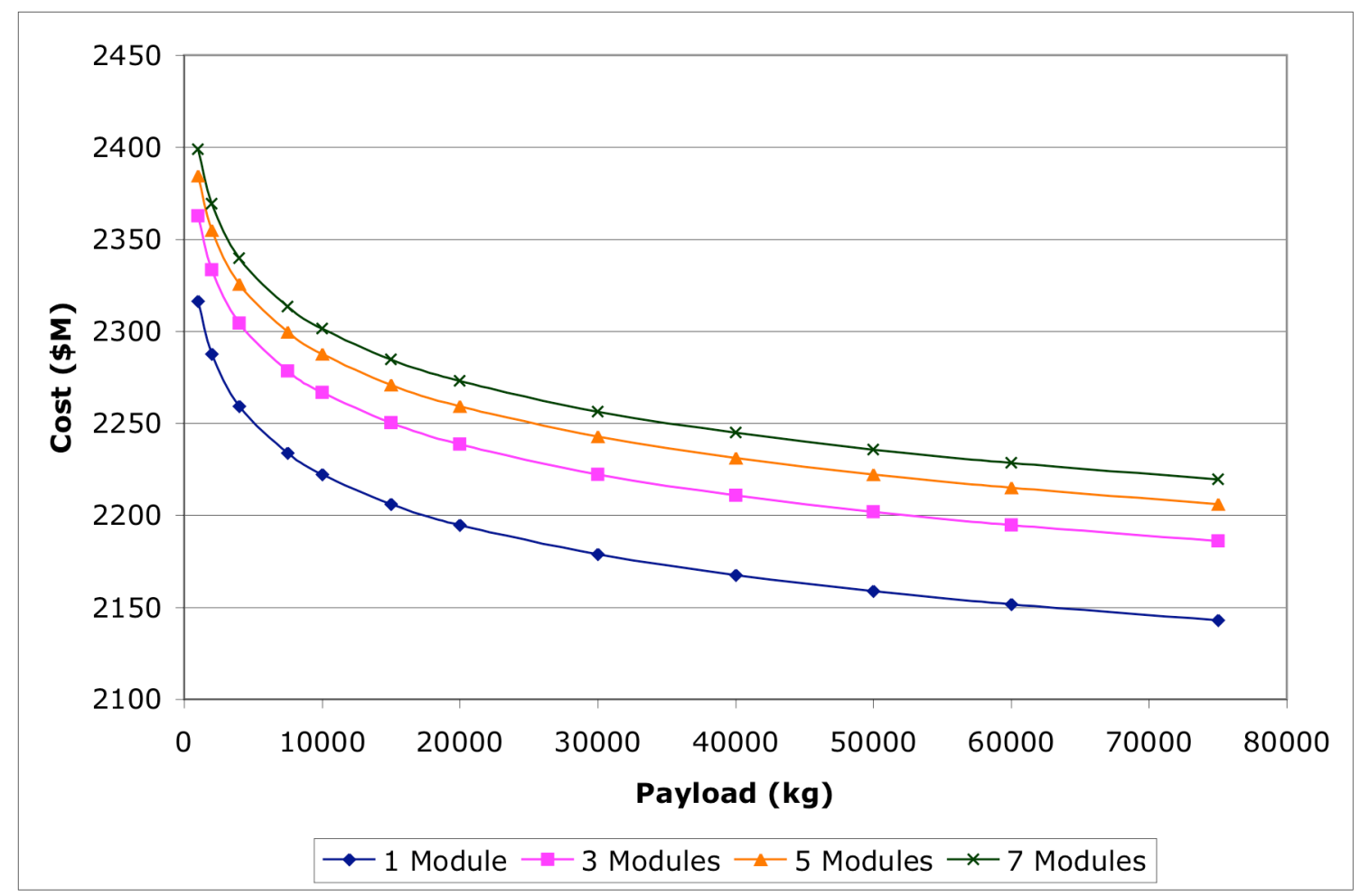

Figure 5.2: Recurring costs $1^{\text {st }}$ stage

The recurring costs increase as the number of modules rises. This is due to the large number of units being produced $(3 \mathrm{x}, 5 \mathrm{x}, 7 \mathrm{x}$, etc.) as opposed to a smaller number for a monolithic stage. The increase in the number of units produced has a positive effect on the cost of individual units, taking advantage of learning curve effects of larger production runs for units beyond the first. While the recurring costs increase given a higher number of modules, they don't increase linearly with the number of modules, thus taking advantage of learning curve effects. 


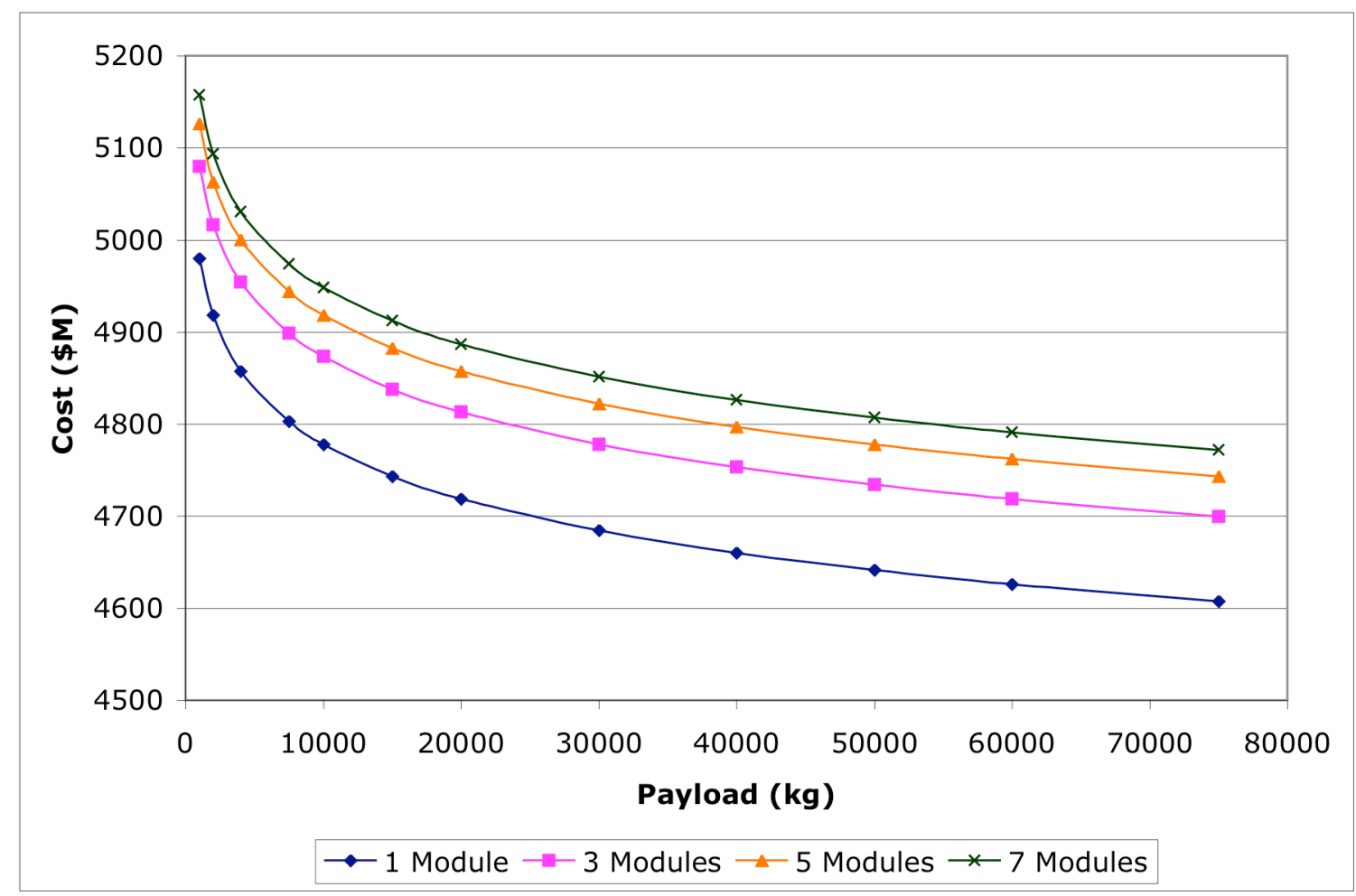

Figure 5.3: Refurbishment costs $1^{\text {st }}$ stage

Refurbishment costs increase as the number of modules in the first stage increases.

While recurring costs are divided over a larger number of units for the same number of flights, which by itself would reduce refurbishment costs, there are more units to refurbish per flight with more modules per vehicle. 


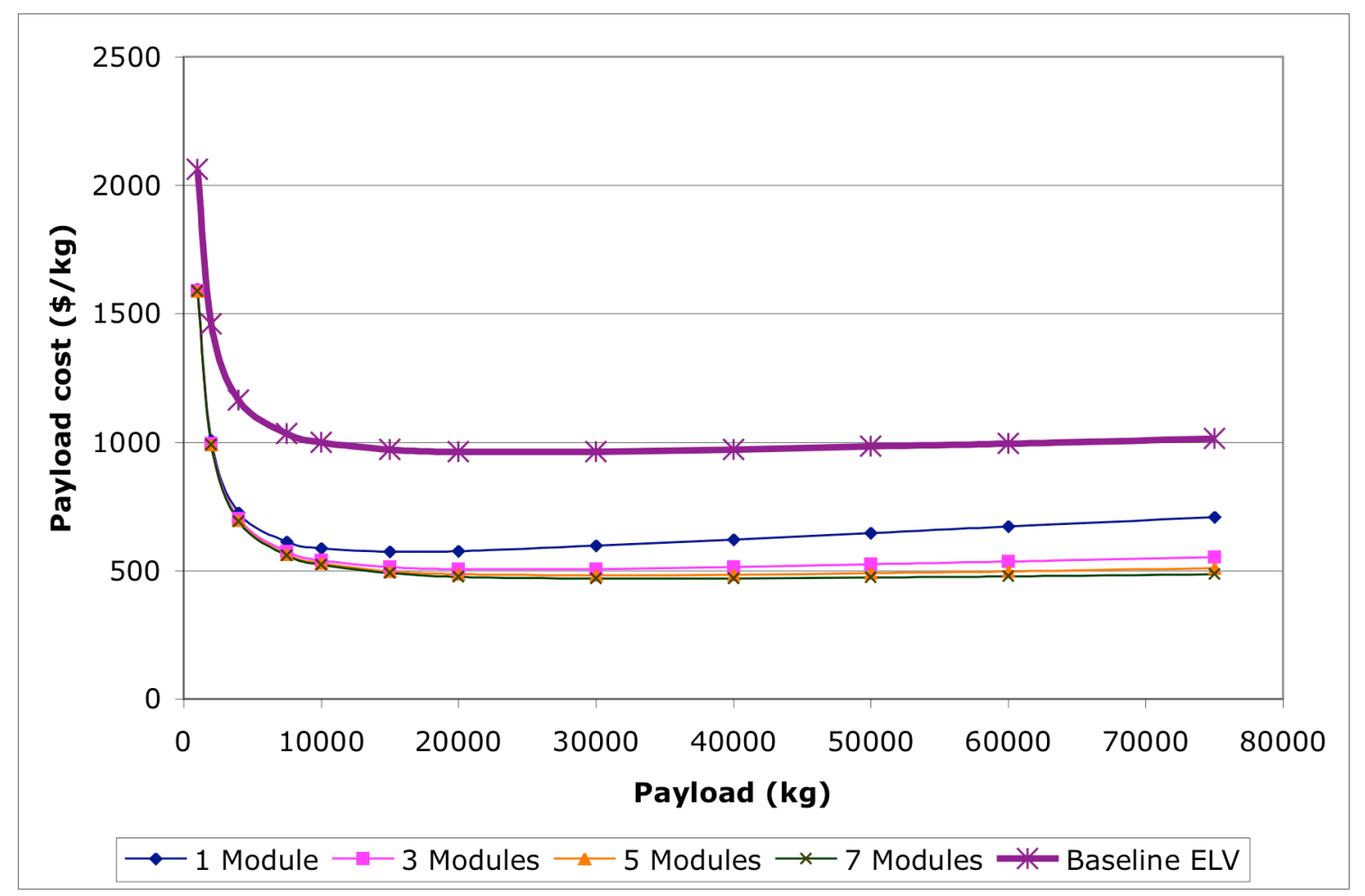

Figure 5.4: Payload costs $1^{\text {st }}$ stage

At low payloads the payload costs are minimized with one module due to the low nonrecurring costs associated with a small vehicle. However as the payload mass increases, the inert mass rises at a rate large enough to make the nonrecurring cost become the cost driver. For multiple module vehicles the nonrecurring costs don't increase as quickly as a monolithic vehicle with rising payload size. Even by adding modules and units to be built thus increasing the recurring costs, the reusable vehicles with modules are still more cost effective than the baseline ELV. However this doesn't include inert mass and operations cost complexities resulting from increased difficulties in developing and assembling multiple modules in the $1^{\text {st }}$ stage. The next sections detail a method to model these increased complexities.

\subsubsection{Inert mass fraction and operations cost complexity factors}


Breaking a monolithic first stage into modules increases the complexity associated with building and assembling the pieces of the stage. Both the cost and inert mass fraction change. To compensate for the increased time and effort spent on assembling modules, a complexity factor is added to the operations cost. Additional dry materials are used in the stage's construction. The inert mass fraction changes as a function of the number of modules used.

\subsubsection{Inert mass fraction complexity factors}

An approximate function of how the inert mass fraction will change as the number of modules changes is found by looking at data from Koelle's TRANSCOST [5]. The net mass fraction, defined by Koelle as the net mass (dry mass plus residuals and gases at cut-off) related to the usable propellant mass, is compared to inert mass fraction. Here the inert mass fraction for a monolithic first stage is 0.078 , previously assigned as the inert mass fraction for cryogenic stages. Koelle's dry mass quantity is comparable to this model's inert mass quantity. Therefore Koelle's net mass fraction is converted to this model's inert mass fraction via the relation below:

$$
\delta=\frac{N M F * m_{\text {prop }}}{m_{\text {gross }}}
$$

Using Koelle's net mass fraction curves for cryogenic stage vehicles [5, p. 31] an extreme point is chosen as unit one to represent one monolithic stage mass. Then the curve is followed to the left to values of $1 / 3,1 / 5$, and $1 / 7$ of the one unit mass to represent the stage broken up into 3, 5, and 7 modules respectively. Values of net mass fraction at 1/3, $1 / 5$ and $1 / 7$ the unit mass are noted to simulate the changing net mass fraction as the stage is broken up into 3, 5 or 7 modules. Then using the equation above these net mass fraction values are converted into inert mass fraction values. 


\begin{tabular}{|c|c|c|c|c|c|c|}
\hline Modules & Unit Mass & \multirow{2}{*}{ NMF } & \multicolumn{2}{|c|}{$\delta(\sqrt[3]{\mathrm{mod}})$} & \multicolumn{2}{c|}{$\delta(\sqrt[4]{\mathrm{mod}})$} \\
\cline { 3 - 7 } & & & ELV & RLV & ELV & RLV \\
\hline 1 & 1 & .090 & .078 & .12 & .078 & .12 \\
\hline 3 & $1 / 3$ & .110 & .112 & .173 & .103 & .158 \\
\hline 5 & $1 / 5$ & .128 & .133 & .205 & .117 & .179 \\
\hline 7 & $1 / 7$ & .135 & .149 & .230 & .127 & .195 \\
\hline
\end{tabular}

Table 5.1: Inert mass fraction as a function of number of modules

By visual inspection, the values of $\delta$ seem to follow a third or fourth root fit as the number of modules increase. Calculating the third and fourth roots of the number of modules represented in the table above shows that the relationship between number of modules, net mass fraction and inert mass fraction follow closely to a fourth root fit. Therefore the inert mass fraction in this cost model will change as a function of the fourth root of the number of modules in the stage.

\subsubsection{Operations cost complexity factors}

An operations cost complexity factor must be added to account for increased complexity of assembling and recovering multiple modules. For the purposes of the two stages vehicle in this model one number will be used to designate the operations cost complexity factor for the whole vehicle. A multiplier of the number of modules is used to determine a complexity factor for operations cost. As mentioned previously the operations cost for a reusable vehicle is $\$ 1.2 \mathrm{M}$ per launch. This is used as the baseline operations cost for a monolithic first stage. There is little published data on operations cost complexity factors involved with multiple booster modules in one stage. Therefore this model considers a range of values and examines the results of each set of values. An assumption is made that if there is a correlation between modules in a stage and increased operations cost, the operations cost is a function of number of modules. Four scenarios are considered: the worst case where the operations cost multiplier is equal to the 
number of modules, the square root of the modules number, the third root of the modules number and the fourth root of the modules number. These values are given in Table 5.2 below with the number of modules and the respective operations cost complexity factor for each scenario.

\begin{tabular}{|c|c|c|c|c|}
\hline Modules & X mods & $\mathrm{f}_{\text {op }} \sqrt{\mathrm{mod}}$ & $\mathrm{f}_{\text {op }} \sqrt[3]{\mathrm{mod}}$ & $\mathrm{f}_{\text {op }} \sqrt[4]{\mathrm{mod}}$ \\
\hline 1 & 1 & 1 & 1 & 1 \\
\hline 3 & 3 & 1.732 & 1.442 & 1.316 \\
\hline 5 & 5 & 2.236 & 1.710 & 1.495 \\
\hline 7 & 7 & 2.646 & 1.913 & 1.627 \\
\hline
\end{tabular}

Table 5.2: Operations complexity factor as a function of number of modules

Using these new inert mass fractions and operations complexity factors as a function of number of modules, each vehicle with first stage modular configurations is graphed at the optimized number of flights per vehicle, given a refurbishment fraction. The refurbishment fraction used here is 0.05 . Trends of payload costs dependent on the number of modules and operations costs configuration are discussed following the graphs. 


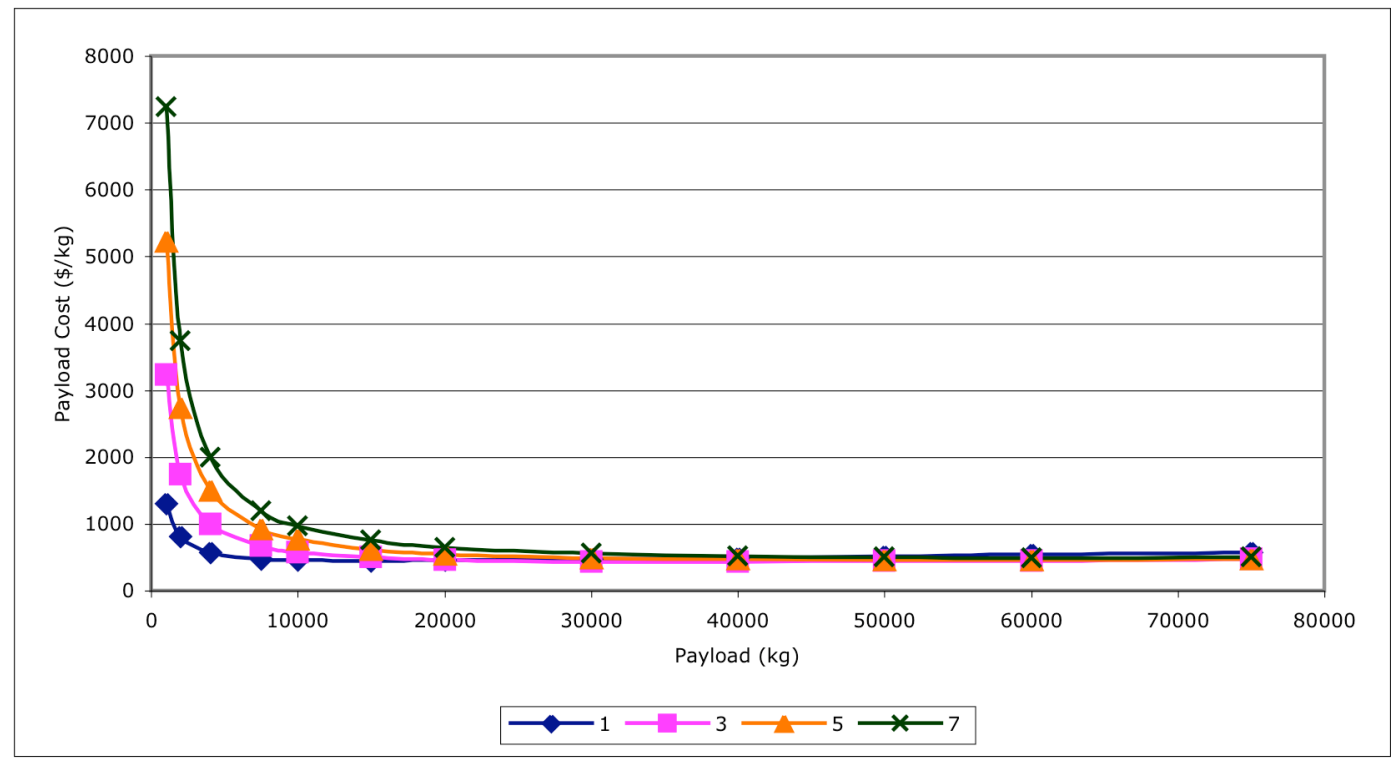

Figure 5.5: Operations cost multiplier (number of modules)

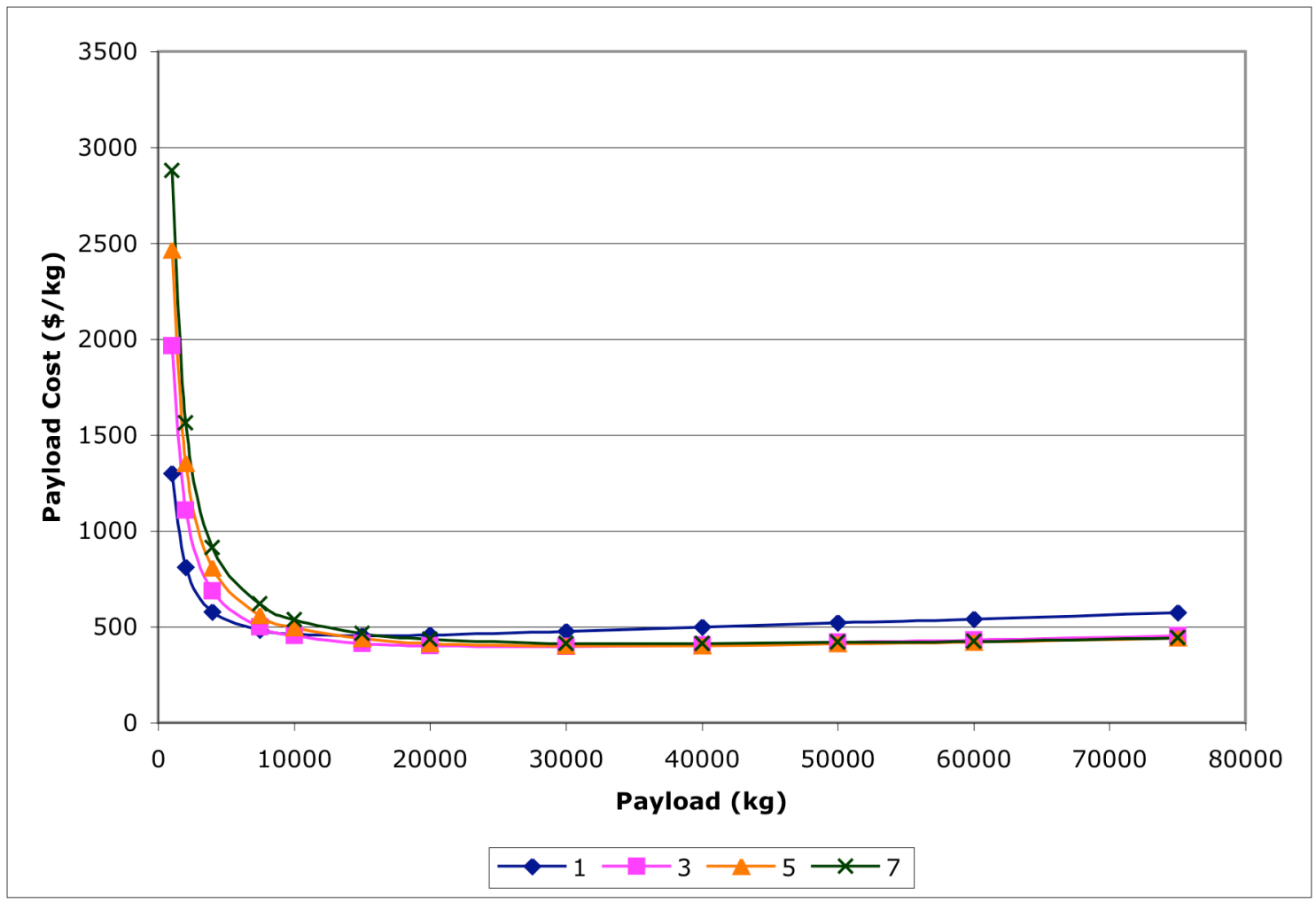

Figure 5.6: Operations cost multiplier (square root number of modules) 


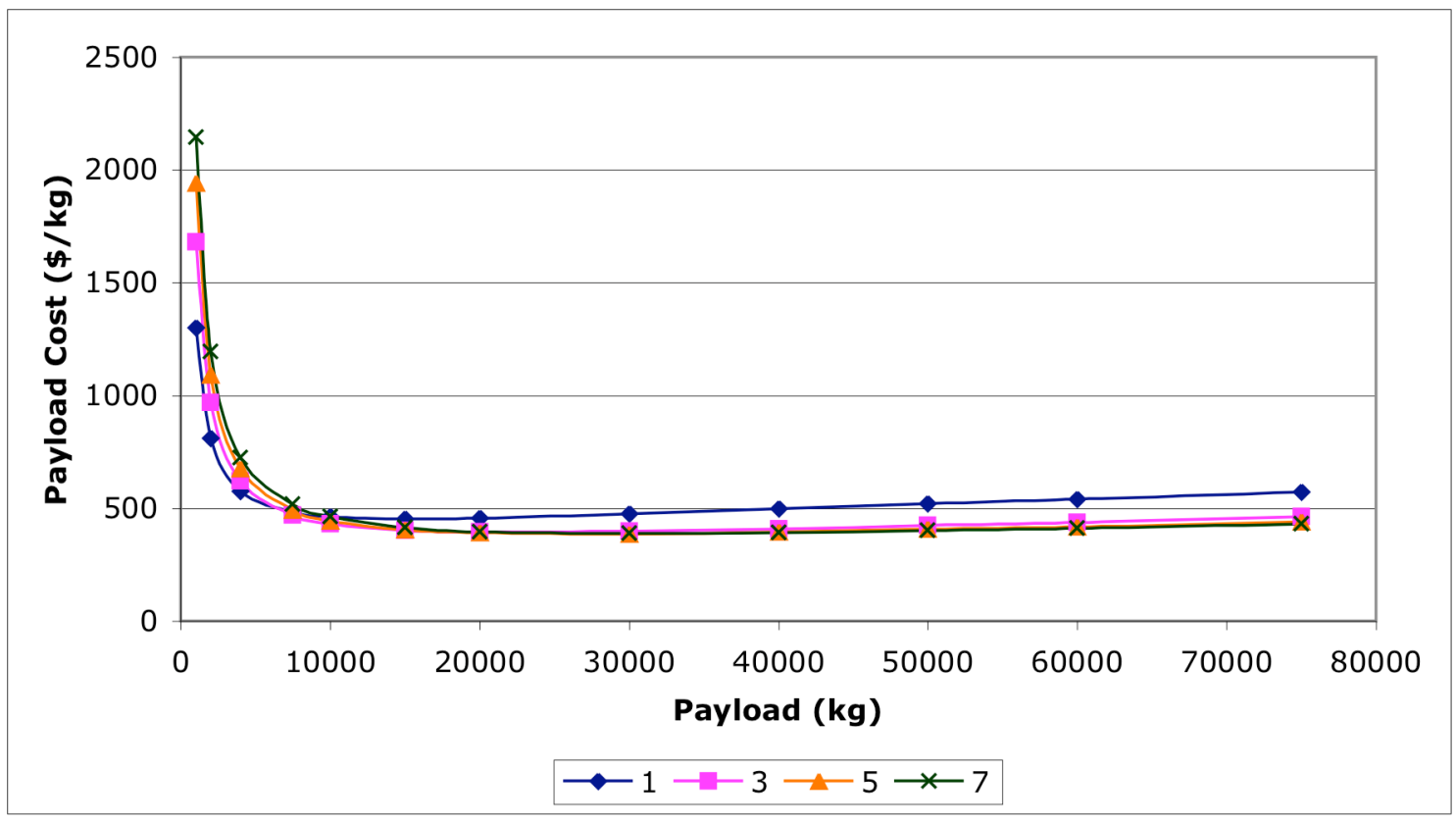

Figure 5.7: Operations cost multiplier (third root number of modules)

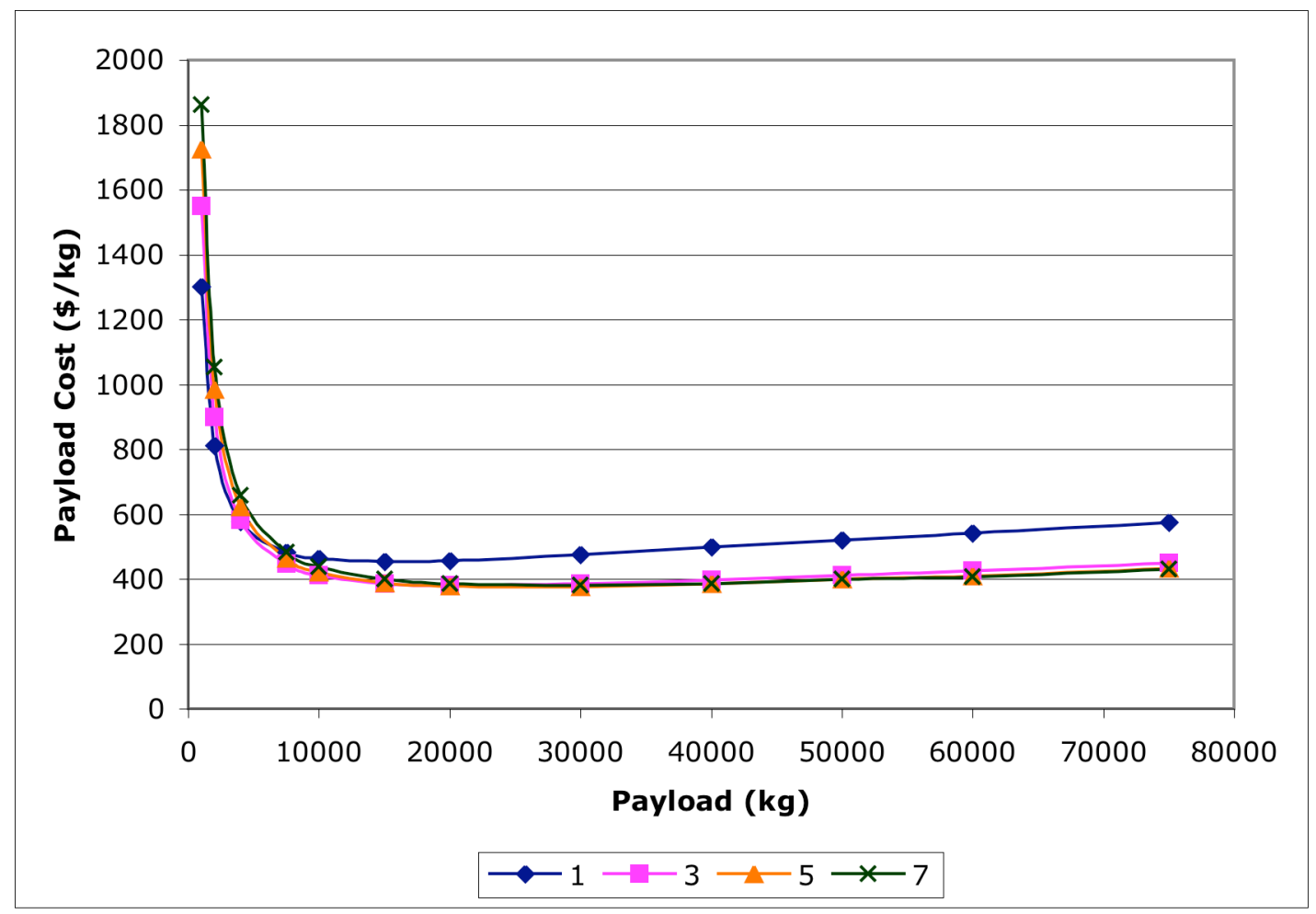

Figure 5.8: Operations cost multiplier (fourth root number of modules) 
Figures 5.5 through 5.8 show the effects of the four operations cost complexity factor scenarios on reusable launch vehicles. On the high extreme (Figure 5.5) the operations cost multiplier is equal to the number of modules. This is a worst-case scenario and in reality it isn't likely that the operations costs are this large, but must be accounted for in a complete survey. At very low payload masses the preflight operations costs dominate due to the large number of flights required for a small payload vehicle. This is due to the high operations costs associated with a large number of flights required for small individual payloads. As the first stage of the vehicle is broken up to into more modules, the total payload costs rises at a rate almost equal to the number of modules for small payloads. This is due to the large operations complexity factors further magnifying the dominating effects of the large flight numbers. At more likely assumptions for an operations complexity factor such as the third or fourth root of the number of modules (Figures 5.7 and 5.8) the operations cost, while still a dominating driver of payload costs for low payload vehicles, doesn't have such an extreme effect on the payload cost.

The crossover points where the payload costs transition from increasing as the number of modules increase to decreasing as the number of modules increase occur at lower payloads as the operations cost complexity factor decreases. For example, in the scenario where the operations cost complexity factor is proportional to the number of modules, payload costs rise as the number of modules increase over a payload range of $1000-20,000 \mathrm{~kg}$. At $30,00 \mathrm{~kg}$ there is a crossover point where payload costs decrease as the module number increases from 1 to 3 and then resume increasing as the module numbers increase from 3 to 7 . For a scenario where the operations costs are lower, such as when the complexity factor is equal to the third root of the module number, this 
crossover point occurs at a lower payload than the previous scenario. Payload costs increase as the module number increases over the small payload range of $1000-4000 \mathrm{~kg}$. At small payloads operations costs are the dominating factor. Hence, small payload vehicles don't see a cost benefit from modularity. In the lower end of the medium payload range of 7,500-15,000 kg, payload costs decrease from 1-3 modules and increase from 3-7 modules. Another crossover point occurs from in the upper end of the medium payload range from 15,000 to $30,000 \mathrm{~kg}$ as payload costs decrease from 1-5 modules, and increases from 5-7 modules. At large payloads of 40,000 kg and more, the payload costs decrease as modules increase from 1-7. Normally without modularity, the nonrecurring costs become very high and are the cost driver at medium to large payloads. With the implementation of modularity the nonrecurring costs receive more cost reducing benefits as the $1^{\text {st }}$ stage is broken down into more modules. The normally very high nonrecurring costs seen at high payloads are reduced with multiple modules, making payload costs decrease with increasing modularity as payloads increase.

The minimum cost point for each operation complexity factor scenario represents the optimal number of modules in the first stage of a vehicle in that scenario. In the worst-case scenario where the operations complexity factor is equal to the number of modules, the minimum cost point occurs around $15,000 \mathrm{~kg}$ with one module. In the third and fourth root scenarios the minimum cost point occurs around 30,000-40,000 kg and five modules. In the worst case scenario one module produces a cost optimum solution because the large operations costs associated with multiple modules overrun the rest of the costs. In the third and fourth root scenarios the operations cost aren't the cost driver, allowing the lower nonrecurring benefits of the multiple modules to be the driver towards 
a minimum payload cost. Based on this finding, the operations cost complexity multiplier used for this study is the fourth root of the number of modules.

\subsubsection{Revised modularity costs}

Based on the selected complexity factors for inert mass fraction and operations cost in the previous section, the principal cost categories are recalculated and shown in Figures 5.9-5.12 below.

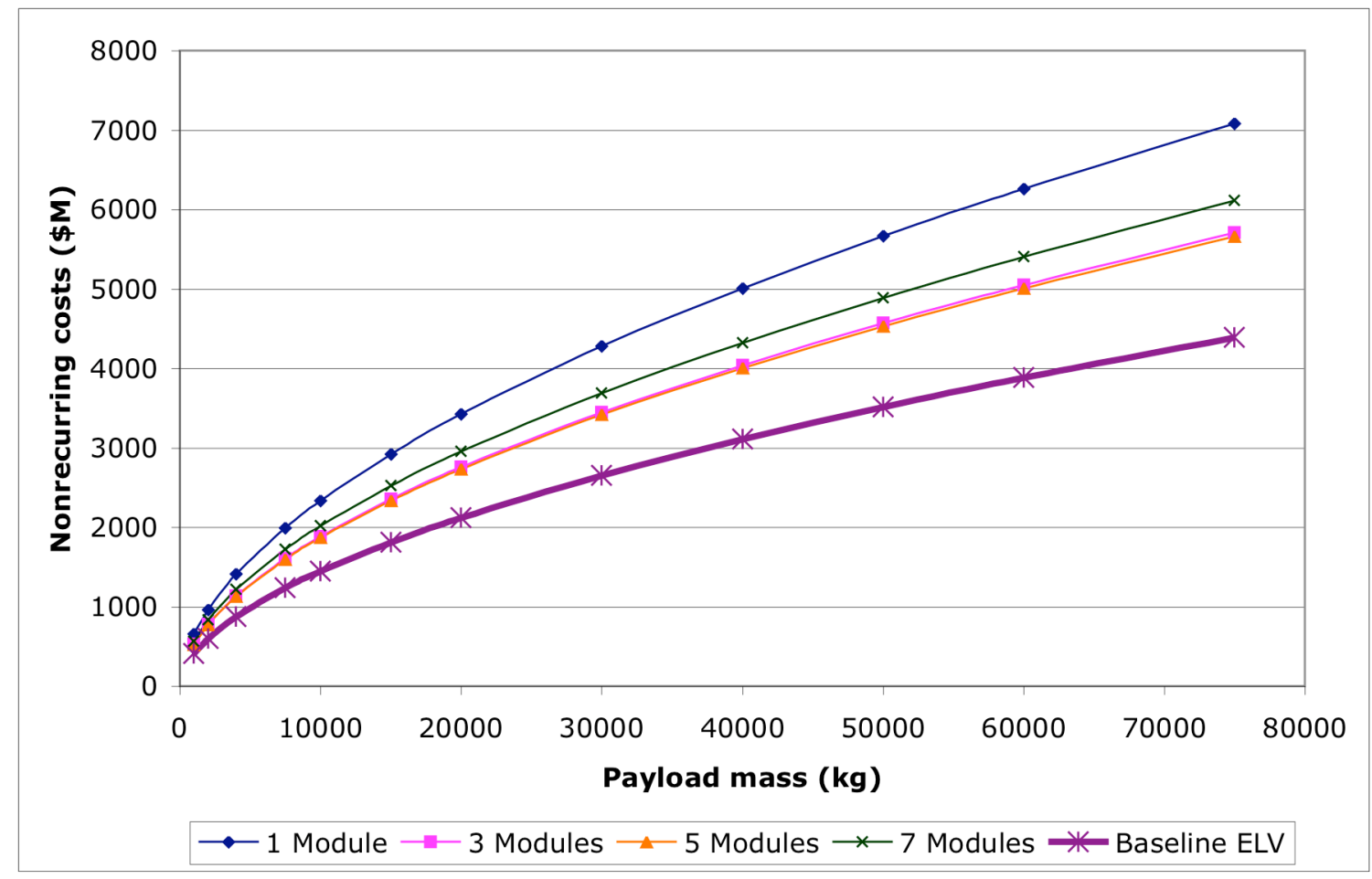

Figure 5.9: Revised nonrecurring costs $1^{\text {st }}$ stage

When the inert mass complexity factor is applied to modularity in the first stage, there is still a cost benefit for nonrecurring costs, but not as much as the initial case. A first stage with multiple modules has lower nonrecurring costs than a monolithic first stage, due to lower inert mass. Since the inert mass fraction is now a function of the number of modules in the first stage, the nonrecurring costs for multiple module stages 
increase as the number of modules per stage increase. The high inert mass fractions make a reusable vehicle with a modular first stage more costly than the baseline ELV.

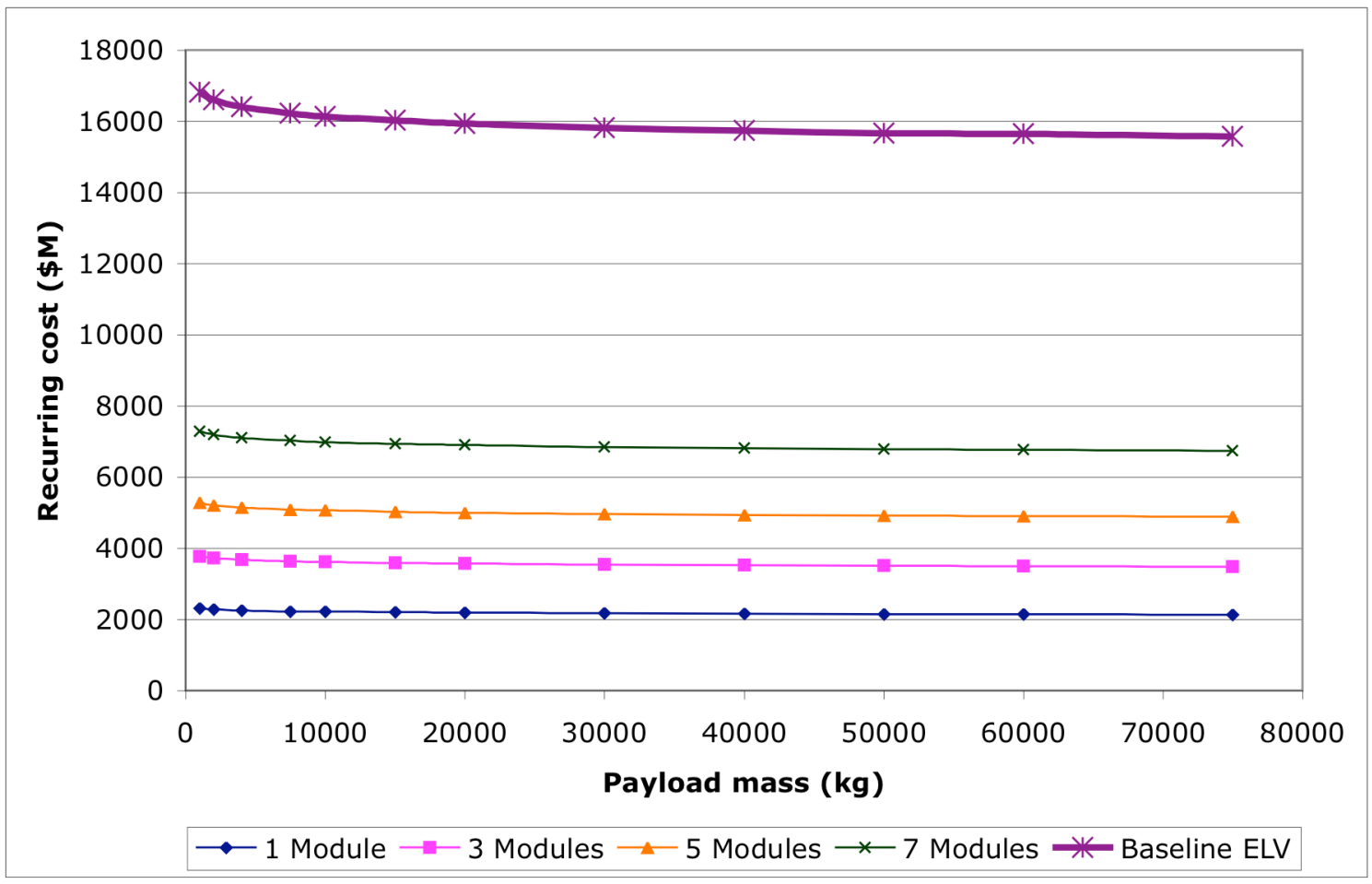

Figure 5.10: Revised recurring costs $1^{\text {st }}$ stage

The recurring costs trend for a reusable vehicle with a modular first stage follows similar trends when inert mass and operations cost complexity factors are implemented. The absolute values of the recurring costs increase due to the increase in first unit production costs due to the higher inert mass fraction for modular vehicles. The RLV first stage with modularity still has lower production costs than the baseline ELV due to the reusability of the modules. The baseline ELV has approximately 7-8x more recurring costs than a monolithic $1^{\text {st }}$ stage RLV, while a RLV with even as many as seven modules on experiences an approximately $2 \mathrm{x}$ increase in recurring costs. 


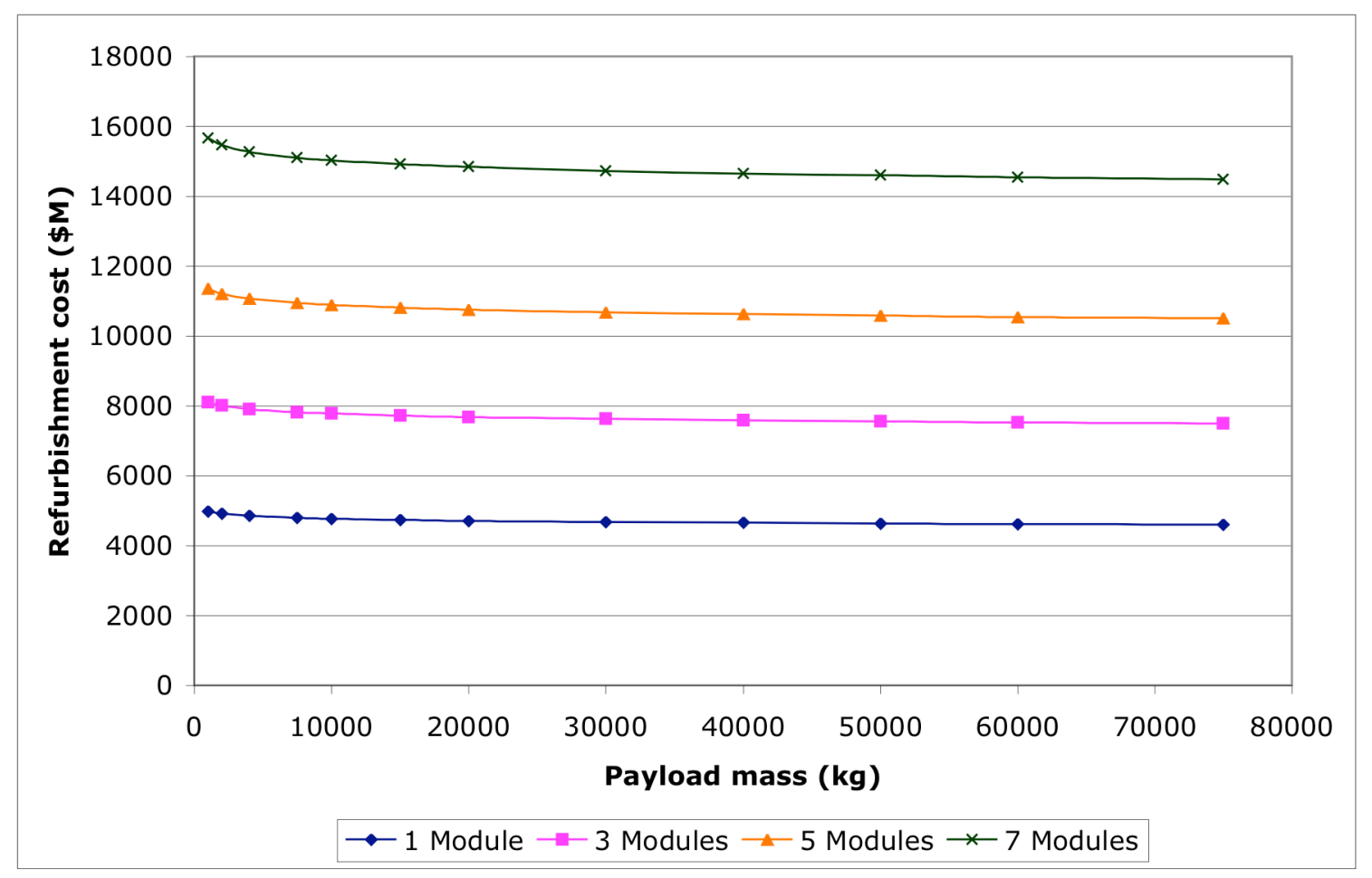

Figure 5.11: Revised refurbishment costs $1^{\text {st }}$ stage

The refurbishment costs experience a large increase when the inert mass fraction complexity factors are implemented. Since each individual module must be recovered and repaired after each flight, there are more units, for each addition of modules per vehicle, to refurbish as opposed to a monolithic $1^{\text {st }}$ stage. 


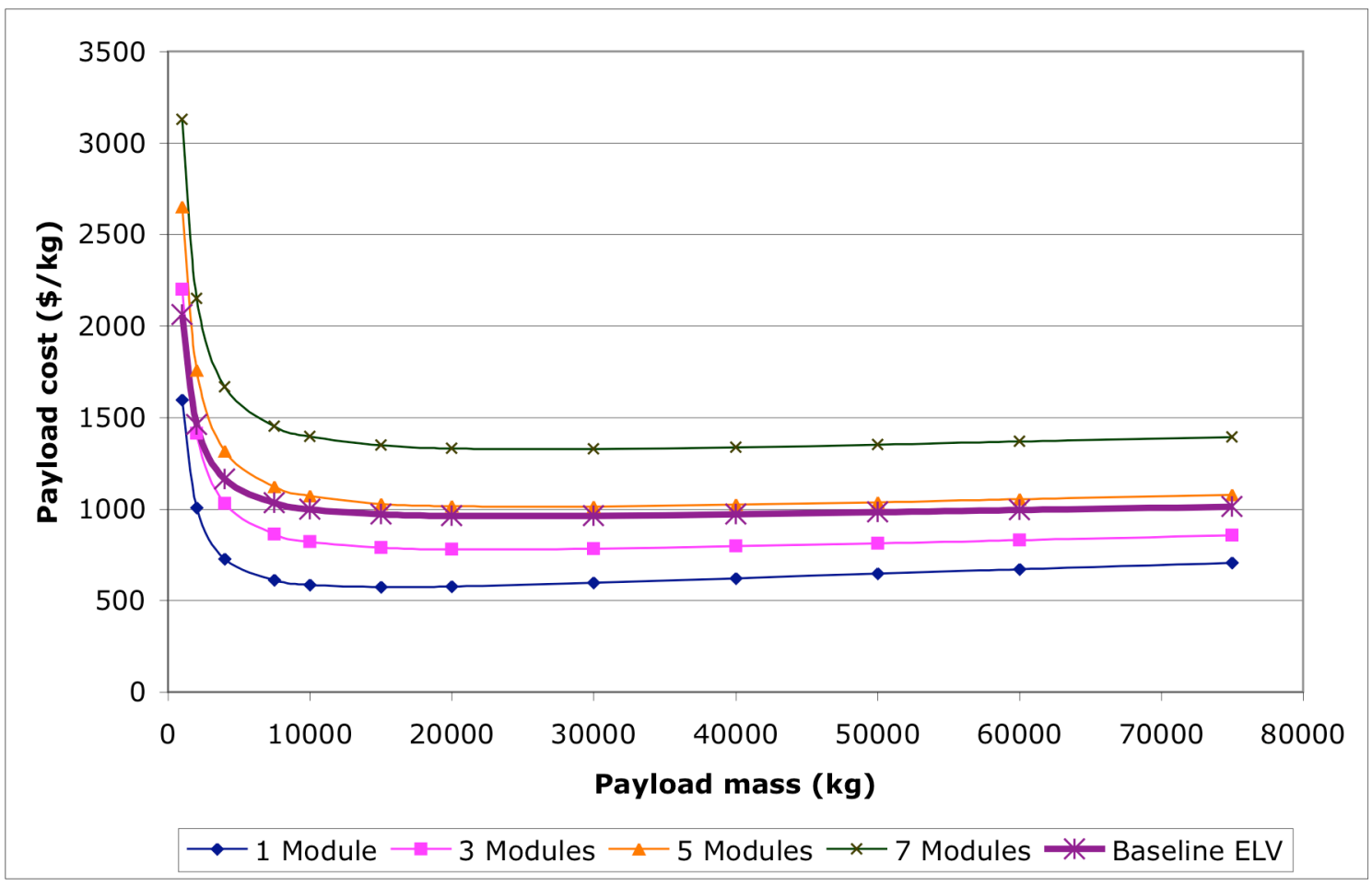

Figure 5.12: Revised payload costs $1^{\text {st }}$ stage

When these component costs are assembled together, the payload costs show the effects of implementing inert mass and operations cost complexity factors on RLV $1^{\text {st }}$ stages with multiple modules. The monolithic reusable vehicle is the most cost efficient. Despite the cost benefits for nonrecurring costs by adding modules to the first stage, the increase in recurring and refurbishment costs are too much to overcome to see cost savings in the overall payload costs. The vehicles with 5 and 7 modules are so complex that they cost more on a per kilogram payload basis than even the baseline ELV with high recurring costs.

\subsubsection{Expendable Launch Vehicles with Modularity}

Figures 5.13-5.15 show the effects of modularity on the costs of an ELV. 


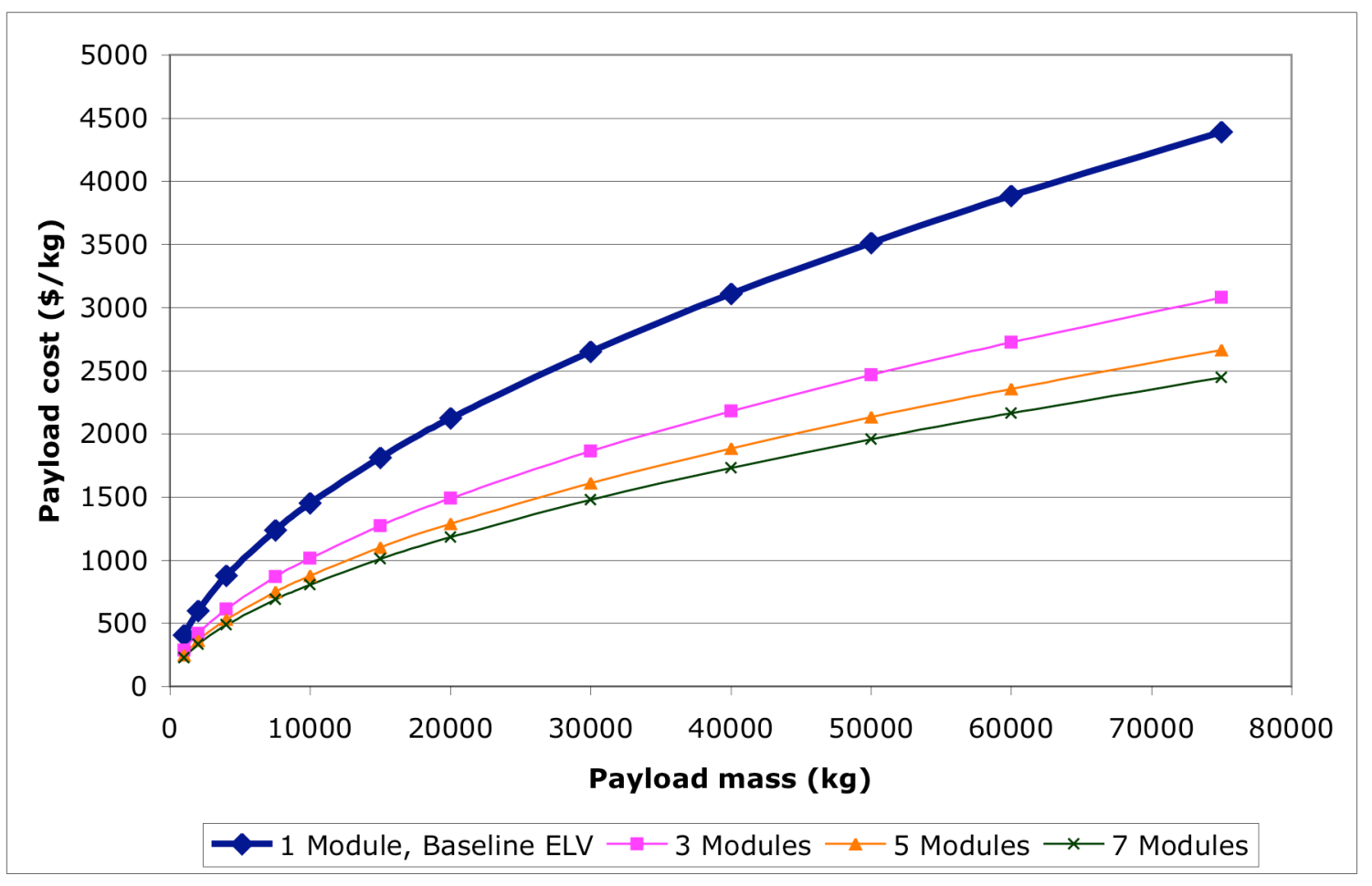

Figure 5.13: Nonrecurring costs ELV $1^{\text {st }}$ stage

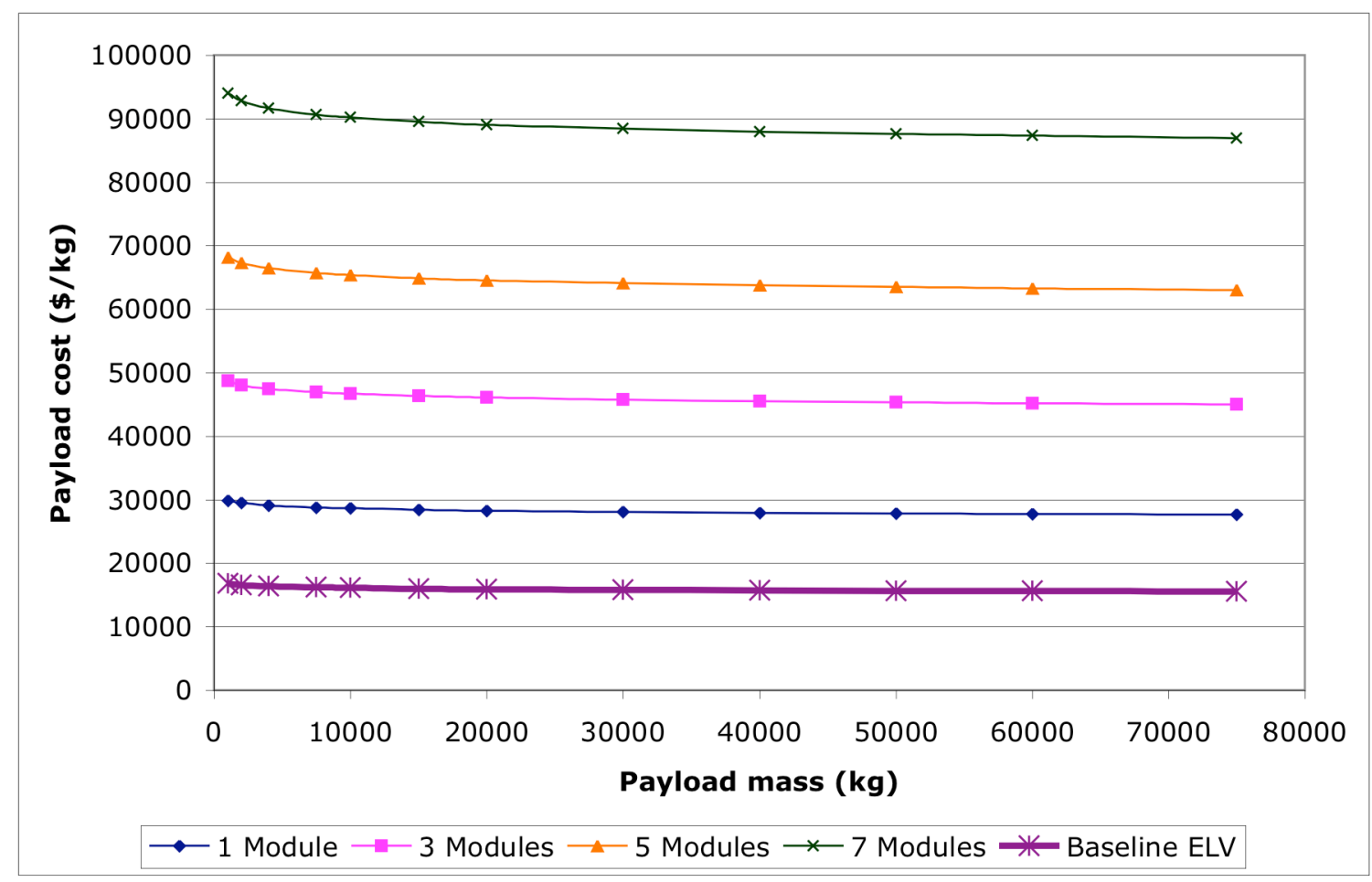

Figure 5.14: Recurring costs ELV $1^{\text {st }}$ stage 


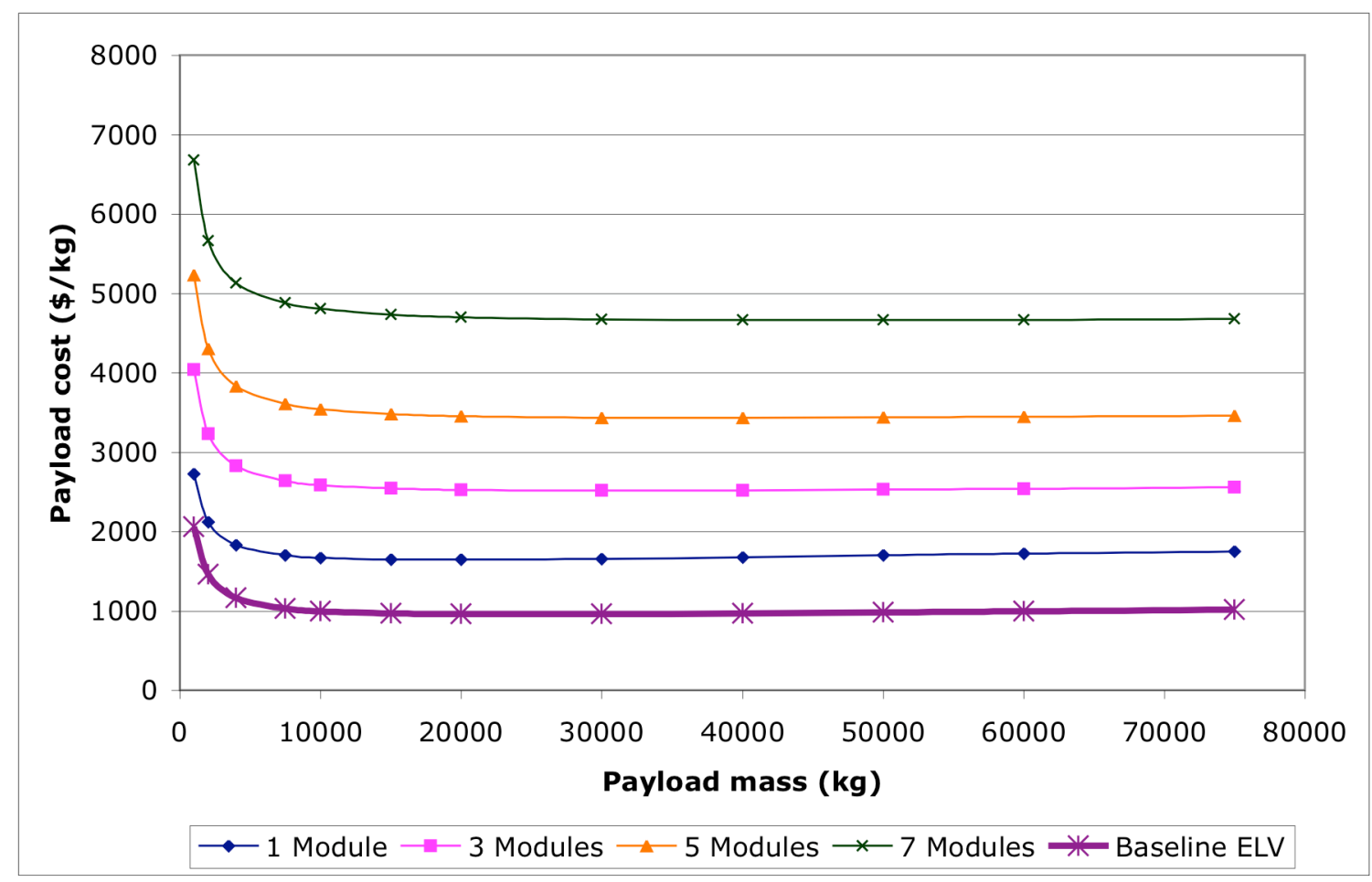

Figure 5.15: Payload costs ELV $1^{\text {st }}$ stage

Across the whole payload size range considered in this study from 1000-75,000 $\mathrm{kg}$ the payload costs increase as the number of modules increase. The overall minimum payload cost for each of the four scenarios occurs with one module at a payload of 20,000 $\mathrm{kg}$. As in the case of the reusable launch vehicles, the high preflight operations costs drive the payload costs at low payloads due to the large number of flights. At higher payloads the recurring costs associated with the large number of expendable vehicles drive the payload costs very high. As more modules are added, the number of expendable unit produced increases, making the recurring costs even higher. The recurring cost increase from producing more modules and throwing them away after each use is too much for the nonrecurring cost savings realized by developing less mass units to overcome. Breaking the first stage into modules is not a viable solution for optimizing costs in expendable launch vehicles. 
At this point it may seem that a reusable vehicle with a monolithic first stage is the most cost optimum solution. However, if the time effects of money are taken into account, the cost benefits that modularity provides nonrecurring costs are improved as will be shown later in this chapter.

\subsubsection{Changes of optimum velocity change split}

Previously the optimum stage 1 velocity change for minimizing cost in a two stage cryogenic reusable launch vehicle was found to be around 0.67 of the total $\Delta \mathrm{V}$. When modularity is implemented the optimum split for velocity change between stage 1 and stage 2 changes. As the number of modules increases, the optimum velocity change in the first stage decreases. This trend is shown below in Figure 5.16.

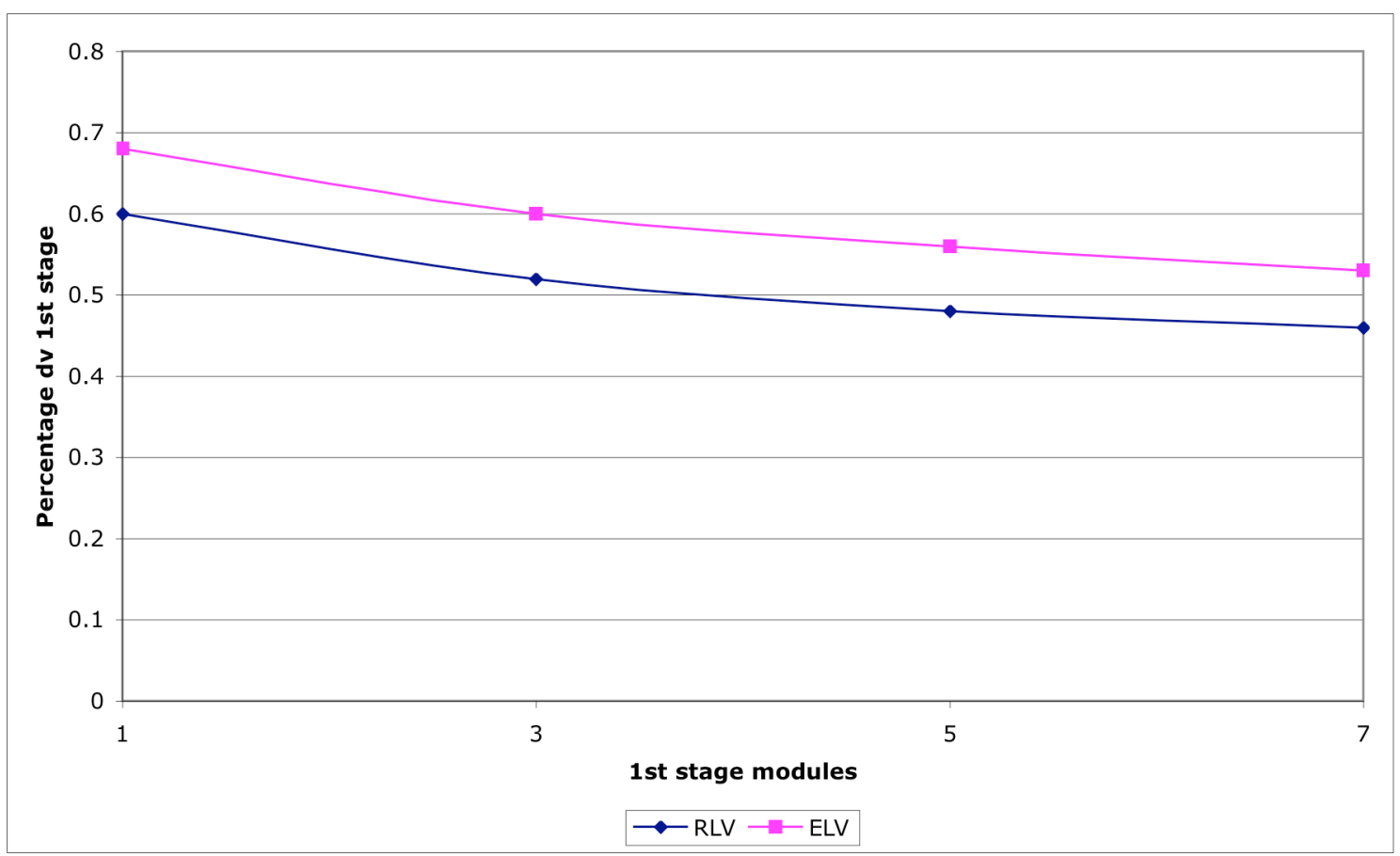

Figure 5.16: Change of optimum $\mathrm{dv}$ in $1^{\text {st }}$ stage with modularity 
As the number of modules increases, the optimum stage 1 velocity change percentage decreases. This is mainly due to the sizing of each individual unit. As more modules are added to the first stage, the size of each unit is made smaller. However the inert mass fraction of the entire stage increases by the factor specified by the inert mass fraction complexity factor. As more modules are added to the stage, there is more inert mass added in the form of structures and wiring to support the integration of multiple modules. With the total inert mass in the first stage increasing, the optimum stage 1 change in velocity decreases. This trend is similar regardless of the reusability of the vehicle. At higher payloads the percentage velocity change in the first stage goes up slightly for each number of modules but the overall decreasing trend remains the same.

For expendable vehicles, the percentage velocity change in the first stage goes up slightly for each similar number of modules as compared to a reusable vehicle, but the overall decreasing trend remains the same.

\subsubsection{Modular staging}

Until this point, when applying modularity to the first stage of launch vehicles, it is assumed that all of the modules in the first stage are firing and burning uniformly at the same time. A complete survey of modularity must include the possibility of a sequence of multiple firings of the first stage module groups. For example 3 modules may be fired in a 2-1 sequence, 5 modules in a 2-2-1 sequence, and 7 modules in a 4-3 or 4-2-1 sequence. At these sequence firings the payload is kept constant. However the module size is expected to shrink as the configuration goes from 7-0 to 4-3 to 4-2-1 because firing modules in additional segments should overshoot the required $\Delta V=9200 \mathrm{~m} / \mathrm{s}$ as 
opposed to firing all modules consecutively. Effectively more stages are being added to the launch vehicle.

To determine the $\Delta V$ produced in the firing of each segment, the standard rocket equation is used:

$$
\Delta V=-g_{0} I_{s p} \ln \left(\frac{M_{f}}{M_{0}}\right)
$$

The initial and final masses for each stage in a two-stage vehicle are broken down as shown in Table 5.3 below.

\begin{tabular}{|c|c|c|}
\hline & Stage 1 & Stage 2 \\
\hline$M_{0}$ & $M_{\text {gross } 1}$ & $M_{\text {gross } 2}$ \\
\hline$M_{f}$ & $M_{\text {gross } 2}+M_{\text {inert } 1}$ & $M_{p L}+M_{\text {inert2 }}$ \\
\hline
\end{tabular}

Table 5.3: Mass breakdowns of two-stage vehicle

Before the $\Delta \mathrm{V}$ values for the segmented firings are calculated, the $\Delta \mathrm{V}$ values for a simultaneous firing must be known. In Table 5.4 below are the $\Delta \mathrm{V}$ values produced in a reusable first stage with the cost optimum $d v$ fractions for a given number of modules in stage 1 .

\begin{tabular}{|l|l|l|}
\hline Stage 1 modules & $d v_{1}$ & $\Delta V_{1}(\mathbf{m} / \mathbf{s})$ \\
\hline 1 & 0.60 & 5520 \\
\hline 3 & 0.52 & 4784 \\
\hline 5 & 0.48 & 4416 \\
\hline 7 & 0.46 & 4232 \\
\hline
\end{tabular}

Table 5.4: Optimal dv values for stage 1 modules 
The $\Delta \mathrm{V}$ for each segmented firing is calculated and the sum is the total $\Delta \mathrm{V}$ produced in stage 1, shown in Table 5.5 below.

\begin{tabular}{|c|c|c|c|c|c|c|c|}
\hline \multirow{2}{*}{ Modules } & \multirow[t]{2}{*}{ Segments } & \multirow{2}{*}{$\begin{array}{c}\text { Initial } \\
\Delta V_{I}\end{array}$} & \multirow{2}{*}{$\begin{array}{c}\text { Resized } \\
\qquad \Delta V_{I}\end{array}$} & \multirow{2}{*}{$\begin{array}{l}\text { Initial } \\
M_{\text {gross } I}\end{array}$} & \multirow{2}{*}{$\begin{array}{c}\text { Resized } \\
\boldsymbol{M}_{\text {gross } I}\end{array}$} & \multicolumn{2}{|c|}{$\begin{array}{l}\$ / \mathrm{kg} \mathrm{PL} \\
@ 1000 \mathrm{~kg}\end{array}$} \\
\hline & & & & & & Initial & Resized \\
\hline 1 & 1 & 5520 & --- & 23310 & --- & --- & --- \\
\hline \multirow{4}{*}{3} & 3 & 4784 & --- & $3 \times 9216$ & --- & --- & --- \\
\hline & 2 & 2531 & 2172 & $2 \times 9216$ & $2 \times 7050$ & & \\
\hline & 1 & 3038 & 2612 & 1x 9216 & 1x 7050 & 1594 & 1550 \\
\hline & Total & 5569 & 4784 & 27648 & 21150 & & \\
\hline \multirow{11}{*}{5} & 5 & 4416 & --- & $5 \times 6054$ & --- & --- & --- \\
\hline & 4 & 3082 & 2676 & $4 \times 6054$ & $4 \times 4683$ & \multirow{3}{*}{1763} & \multirow{3}{*}{1721} \\
\hline & 1 & 2070 & 1740 & $1 \times 6054$ & $1 \times 4683$ & & \\
\hline & Total & 5153 & 4416 & 30270 & 15875 & & \\
\hline & 3 & 2074 & 1738 & $3 \times 6054$ & $3 \times 4640$ & \multirow{3}{*}{1763} & \multirow{3}{*}{1720} \\
\hline & 2 & 3079 & 2678 & $2 \times 6054$ & $2 \times 4640$ & & \\
\hline & Total & 5753 & 4416 & 30270 & 23200 & & \\
\hline & 2 & 1263 & 915 & $2 \times 6054$ & $2 \times 4373$ & \multirow{4}{*}{1763} & \multirow{4}{*}{1711} \\
\hline & 2 & 2073 & 1843 & $2 \times 6054$ & $2 \times 4373$ & & \\
\hline & 1 & 2070 & 1658 & $1 \times 6054$ & $1 \times 4373$ & & \\
\hline & Total & 5406 & 4416 & 30270 & 21865 & & \\
\hline \multirow{14}{*}{7} & 7 & 5428 & --- & $7 \times 4650$ & --- & --- & --- \\
\hline & 6 & 3297 & 2895 & $6 \times 4650$ & $6 \times 3649$ & \multirow{3}{*}{1898} & \multirow{3}{*}{1857} \\
\hline & 1 & 1598 & 1337 & $1 \times 4650$ & $1 \times 3649$ & & \\
\hline & Total & 4895 & 4232 & 32550 & 25543 & & \\
\hline & 4 & 1889 & 1556 & $4 \times 4650$ & $4 \times 3534$ & \multirow{3}{*}{1898} & \multirow{3}{*}{1853} \\
\hline & 3 & 3072 & 2676 & $3 \times 4650$ & $3 \times 3534$ & & \\
\hline & Total & 4961 & 4232 & 32550 & 24738 & & \\
\hline & 5 & 2534 & 2132 & $5 \times 4650$ & $5 \times 3496$ & \multirow{3}{*}{1898} & \multirow{3}{*}{1851} \\
\hline & 2 & 2490 & 2100 & $2 \times 4650$ & $2 \times 3496$ & & \\
\hline & Total & 5024 & 4232 & 32550 & 24472 & & \\
\hline & 4 & 1889 & 1463 & $4 \times 4650$ & $4 \times 3299$ & \multirow{4}{*}{1898} & \multirow{4}{*}{1843} \\
\hline & 2 & 1781 & 1532 & $2 \times 4650$ & $2 \times 3299$ & & \\
\hline & 1 & 1598 & 1238 & $1 \times 4650$ & 1x 3299 & & \\
\hline & Total & 5267 & 4232 & 32550 & 23093 & & \\
\hline
\end{tabular}

Table 5.5: Segmented module firings for stage 1 
The values shown above are for a reusable vehicle with various segmented firing patterns in a first stage with multiple modules at a payload of $1000 \mathrm{~kg}$. It is only necessary to do this analysis at one payload value because the mass ratio stays constant. Therefore the $M_{\text {gross }}$, both initial and resized, change proportionally with payload. Using the rocket equation, the $\Delta V$ produced by each segmented firing listed is calculated. The initial $\Delta V$ is the value produced by each segmented firing with the modules sized as they were for a monolithic first stage. As expected, the total $\Delta V$ produced for segmented firings overshoots the $\Delta V_{l}$ required for stage 1 . Therefore the individual module is resized and made smaller until the resized $\Delta V_{l}$ is equal to the $\Delta V_{l}$ required for stage 1. Note the $\Delta V_{l}$ required for stage 1 changes depending on the number of modules as detailed above. The initial $M_{\text {gross }}$ is the size of each individual module, and is multiplied by the number of modules indicated in the segments column. The resized $M_{\text {grossl }}$ is the size of each unit after the $\Delta V_{l}$ for each segmented firing is recalculated, so the total $\Delta V_{l}$ is equal to the $\Delta V_{l}$ required for the first stage. The initial $\$ / \mathrm{kg}$ PL is the cost per kilogram payload at $1000 \mathrm{~kg}$ payload at the initial gross mass. The resized $\$ / \mathrm{kg}$ PL is the cost per kilogram payload at $1000 \mathrm{~kg}$ payload at the resized gross mass.

As the segmented module firings are broken down from all modules firing simultaneously to multiple firings, the mass of each module unit goes down. As indicated by the resized $M_{\text {gross }}$ column, multiple modules fired in multiple segments (2-21, 4-2-1, etc.) drive the overall size of each unit down. In turn, this drives the payload 
cost down. If it is proven technologically feasible, it is advantageous to fire multiple modules in segments to reach a cost optimum solution. It should be noted that multiple firings add complexity factors that may offset the mass and cost savings seen here.

\subsection{Cost discounting}

Cost discounting is the traditional analysis technique to incorporate the opportunity costs of investing, wherein deferring expenditures is preferable to making payments in the early years of a program. Particularly for commercial launch vehicle programs, the traditional high nonrecurring costs of a space vehicle have prevented the levels of return on investment currently expected by venture capitalists. A discount rate of $25-30 \%$ is typical for risky investments such as a launch vehicle [10]. Incorporating the effects of cost discounting at various interest rates will further increase the bias towards smaller and more inexpensive launch vehicles flown in greater numbers, as well as increase the attractiveness of modular design concepts.

\subsubsection{Cost Discounting Methodology}

To integrate the effects of cost discounting into the primary model, the program is set on a timeline of 25 years, beginning in 2005 and ending in 2029. The total program cost over this time period is divided into 4 subdivisions of when the majority of each cost subdivision is expected to occur. Nonrecurring costs occur in year 1-5 (2005-2009), production costs occur in year 3-7 (2007-2011), operations costs occur in year 6-25 (2010-2029) and refurbishment costs for RLVs occur in year 6-25 (2010-2029). The operations and refurbishment costs timeline reflect the 20 -year flight period of the program. 
Since costs are rarely constant from year to year in a multiyear program, the costs must be spread over their respective periods. This is achieved using a beta function. A beta function determines the program costs in each year dependent on the fraction of total time that has passed. The two factors that determine the general shape of a beta function are cost fraction $(c f)$ and peakedness $(p f)$. The cost fraction is the fraction of the total cost to be to be spent when $50 \%$ of the time has elapsed and the peakedness determines the maximum annual cost. The cost fraction for any given year (CF) is calculated using the following:

$C F=a\left(10 t f^{2}-20 t f^{3}+10 t f^{4}\right)+b\left(10 t f^{3}-20 t f^{4}+10 t f^{5}\right)+\left(5 t f^{4}-4 t f^{5}\right)$

where:

$a=\frac{p(c f-0.8125)+c f-0.1825}{0.625}$

$b=\frac{p(0.8125-c f)}{0.3125}$

$t f=\frac{12(1+\text { year }- \text { begin })}{\text { months }}$

months $=12($ end - begin +1$)$

begin=beginning year

end=ending year

year=current year

The annual cost and cumulative cost are determined by:

Annual Cost $=\left(C F-C F_{\text {previous }}\right) *$ total cost

Cumulative Cost $=C F^{*}$ total cost 
Once the costs are spread over the respective years in which they are incurred, the time effects of money must be taken into account by calculating the present value (PV) of each cost. Present value is the amount that a future sum of money is worth today given a rate of return.

$C_{i}=C_{i+n}(1+r)^{-n}$

where:

$C_{i}=$ present value in year 0

$C_{i+n}=$ future value in year $n$

$r=$ discount rate

$n=$ year number in program

The discount rate is an interest rate used in determining the present value of future cash flows. For this study, the discount rate is set at various rates of return to reach the revenue required for private investors in the program to achieve their expected rate of return on their money. Return rates (RR) of $10 \%, 25 \%, 50 \%, 75 \%$ and $100 \%$ are modeled. $0 \%$ is also modeled as a control group where there is no private investment money involved.

\subsubsection{Cost discounting application}

To fully understand the effect of various discount rates on different RLV configurations, a TSTO RLV with the cost optimum pairings of number of flights per vehicle and refurbishment fraction is modeled over the payload range from $1000 \mathrm{~kg}$ to $75000 \mathrm{~kg}$ for each discount rate. The first and second stages are identical with an Isp of 425 seconds and inert mass fraction of 0.078 . The optimum staging velocity for 
minimizing cost is calculated at each point. For each case the total cost is broken down into the four cost categories: nonrecurring, production, refurbishment and operations costs. These four subcategories are cost spread over the life of the program using the beta function and then the present value of each cost incurred in future years is calculated, using the selected discount rate. After cost spreading and present value calculations are performed, the costs and their present values are totaled. An example is displayed below. In this example the return rate is set at $50 \%$, the number of flights per vehicle is 43 and the refurbishment fraction is $5 \%$.

\begin{tabular}{|c|c|c|c|c|c|}
\hline Total Costs & 9021.2 & & & PV (2004) & PV (2004) \\
\hline Year & Costs \$M & Flights & Revenue & Costs & Revenue \\
\hline 2005 & 113.59 & & & 75.72 & \\
\hline 2006 & 508.97 & & & 226.20 & \\
\hline 2007 & 809.77 & & & 239.93 & \\
\hline 2008 & 928.82 & & & 183.47 & \\
\hline 2009 & 704.28 & & & 92.74 & \\
\hline 2010 & 691.96 & 100.00 & 1000.00 & 60.74 & 87.79 \\
\hline 2011 & 365.81 & 100.00 & 1000.00 & 21.41 & 58.52 \\
\hline 2012 & 272.11 & 100.00 & 1000.00 & 10.61 & 39.01 \\
\hline 2013 & 272.11 & 100.00 & 1000.00 & 7.07 & 26.01 \\
\hline 2014 & 272.11 & 100.00 & 1000.00 & 4.71 & 17.34 \\
\hline 2015 & 272.11 & 100.00 & 1000.00 & 3.14 & 11.56 \\
\hline 2016 & 272.11 & 100.00 & 1000.00 & 2.09 & 7.70 \\
\hline 2017 & 272.11 & 100.00 & 1000.00 & 1.39 & 5.13 \\
\hline 2018 & 272.11 & 100.00 & 1000.00 & 0.93 & 3.42 \\
\hline 2019 & 272.11 & 100.00 & 1000.00 & 0.62 & 2.28 \\
\hline 2020 & 272.11 & 100.00 & 1000.00 & 0.41 & 1.52 \\
\hline 2021 & 272.11 & 100.00 & 1000.00 & 0.27 & 1.01 \\
\hline 2022 & 272.11 & 100.00 & 1000.00 & 0.18 & 0.67 \\
\hline 2023 & 272.11 & 100.00 & 1000.00 & 0.12 & 0.45 \\
\hline 2024 & 272.11 & 100.00 & 1000.00 & 0.08 & 0.30 \\
\hline 2025 & 272.11 & 100.00 & 1000.00 & 0.05 & 0.20 \\
\hline 2026 & 272.11 & 100.00 & 1000.00 & 0.03 & 0.13 \\
\hline 2027 & 272.11 & 100.00 & 1000.00 & 0.02 & 0.08 \\
\hline 2028 & 272.11 & 100.00 & 1000.00 & 0.01 & 0.05 \\
\hline 2029 & 272.11 & 100.00 & 1000.00 & 0.01 & 0.03 \\
\hline Total & 9021.20 & 2000.00 & 20000.00 & 932.07 & 263.29 \\
\hline
\end{tabular}


An arbitrary value of $\$ 1000 \mathrm{M}$ revenue per year is selected. To determine the amount of revenue required to provide investors their expected rate of return, the present value of the revenue must equal the present value of the costs. The correct value of revenue required to give an investors their expected rate of return is calculated by setting the present value of the revenue (shown in the right hand column) equal to the present value of the costs shown in the column $2^{\text {nd }}$ from the right. Using Excel's Solver function a value for revenue per year is entered to make the two right hand columns equal as shown below.

\begin{tabular}{rrrrrr}
$\begin{array}{c}\text { Total Costs } \\
\text { Year }\end{array}$ & $\begin{array}{c}9021.2 \\
\text { Costs \$M }\end{array}$ & Flights & \multicolumn{3}{c}{$\begin{array}{c}\text { NPV (2004) } \\
\text { NPV (2004) } \\
\text { Revenue \$M } \\
\text { Costs \$M }\end{array}$ Revenue \$M } \\
2006 & 113.59 & & & 75.72 & \\
2007 & 508.97 & & & 226.20 & \\
2008 & 909.77 & & & 239.93 & \\
2009 & 704.28 & & & 183.47 & \\
2010 & 691.96 & 100.00 & 3540.04 & 60.74 & 310.78 \\
2011 & 365.81 & 100.00 & 3540.04 & 21.41 & 207.19 \\
2012 & 272.11 & 100.00 & 3540.04 & 10.61 & 138.12 \\
2013 & 272.11 & 100.00 & 3540.04 & 7.07 & 92.08 \\
2014 & 272.11 & 100.00 & 3540.04 & 4.71 & 61.38 \\
2015 & 272.11 & 100.00 & 3540.04 & 3.14 & 40.92 \\
2016 & 272.11 & 100.00 & 3540.04 & 2.09 & 27.28 \\
2017 & 272.11 & 100.00 & 3540.04 & 1.39 & 18.18 \\
2018 & 272.11 & 100.00 & 3540.04 & 0.93 & 12.12 \\
2019 & 272.11 & 100.00 & 3540.04 & 0.62 & 8.08 \\
2020 & 272.11 & 100.00 & 3540.04 & 0.41 & 5.38 \\
2021 & 272.11 & 100.00 & 3540.04 & 0.27 & 3.59 \\
2022 & 272.11 & 100.00 & 3540.04 & 0.18 & 2.39 \\
2023 & 272.11 & 100.00 & 3540.04 & 0.12 & 1.59 \\
2024 & 272.11 & 100.00 & 3540.04 & 0.08 & 1.06 \\
2025 & 272.11 & 100.00 & 3540.04 & 0.05 & 0.70 \\
2026 & 272.11 & 100.00 & 3540.04 & 0.03 & 0.47 \\
2027 & 272.11 & 100.00 & 3540.04 & 0.02 & 0.31 \\
2028 & 272.11 & 100.00 & 3540.04 & 0.01 & 0.21 \\
2029 & 272.11 & 100.00 & 3540.04 & 0.01 & 0.14 \\
Total & 9021.20 & 2000.00 & 70800.85 & 932.07 & 932.07
\end{tabular}




\section{Table 5.7: Solved for breakeven costs and revenue at $50 \%$ return rate}

The new value for revenue, $\$ 3540 \mathrm{M}$, is the amount that should charged on a per year basis, equal to about $\$ 36 \mathrm{M}$ per flight, to give investors their expected return on their money. A similar analysis is done for each return rate for each payload for each refurbishment fraction. The results are compiled and examined to understand the vehicle payload mass at which a cost minimum is reached for each return rate.

The payload values at which a cost minimum is reached are displayed below for each return rate considered.

\begin{tabular}{|l|l|l|l|l|l|l|c|}
\hline \multicolumn{2}{|c|}{ RR (x100\%) } & \multicolumn{1}{c|}{$\mathbf{0 . 1}$} & \multicolumn{1}{c|}{$\mathbf{0 . 2 5}$} & $\mathbf{0 . 5}$ & $\mathbf{0 . 7 5}$ & \multicolumn{1}{c|}{$\mathbf{1}$} & $\mathbf{0}$ \\
\hline $\begin{array}{l}\text { Payload } \\
\text { at } \\
\text { minimum } \\
\text { cost (kg) }\end{array}$ & RLV & 7500 & 4000 & 2000 & 1250 & 750 & 15000 \\
\cline { 2 - 7 } & ELV & 10000 & 4000 & 2000 & 1500 & 1000 & 20000 \\
\hline
\end{tabular}

Table 5.8: Payload values at minimum cost for various rates of return 


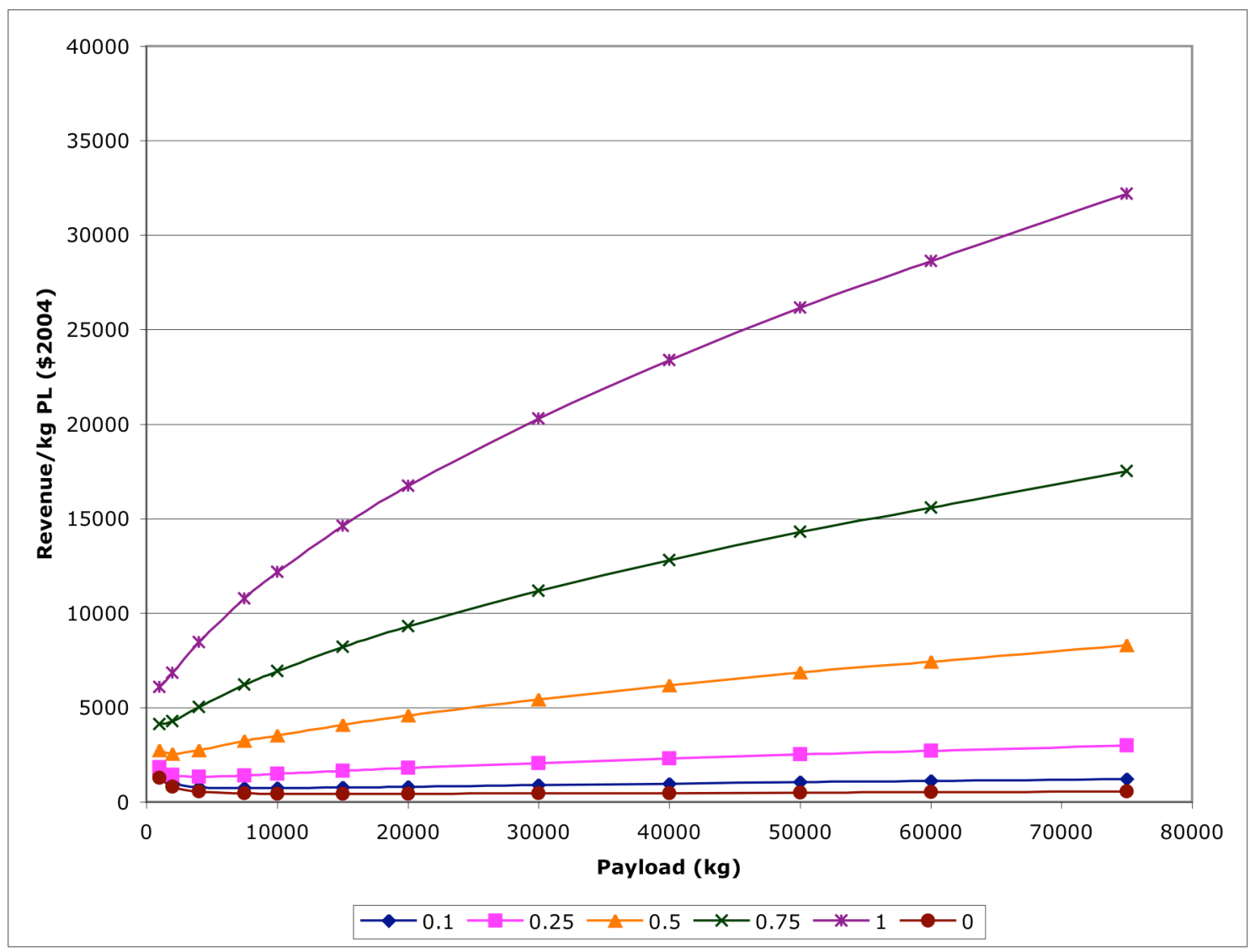

Figure 5.17: Revenue required at various rates of return for small refurbishment fraction $(\mathbf{0 . 0 5})$

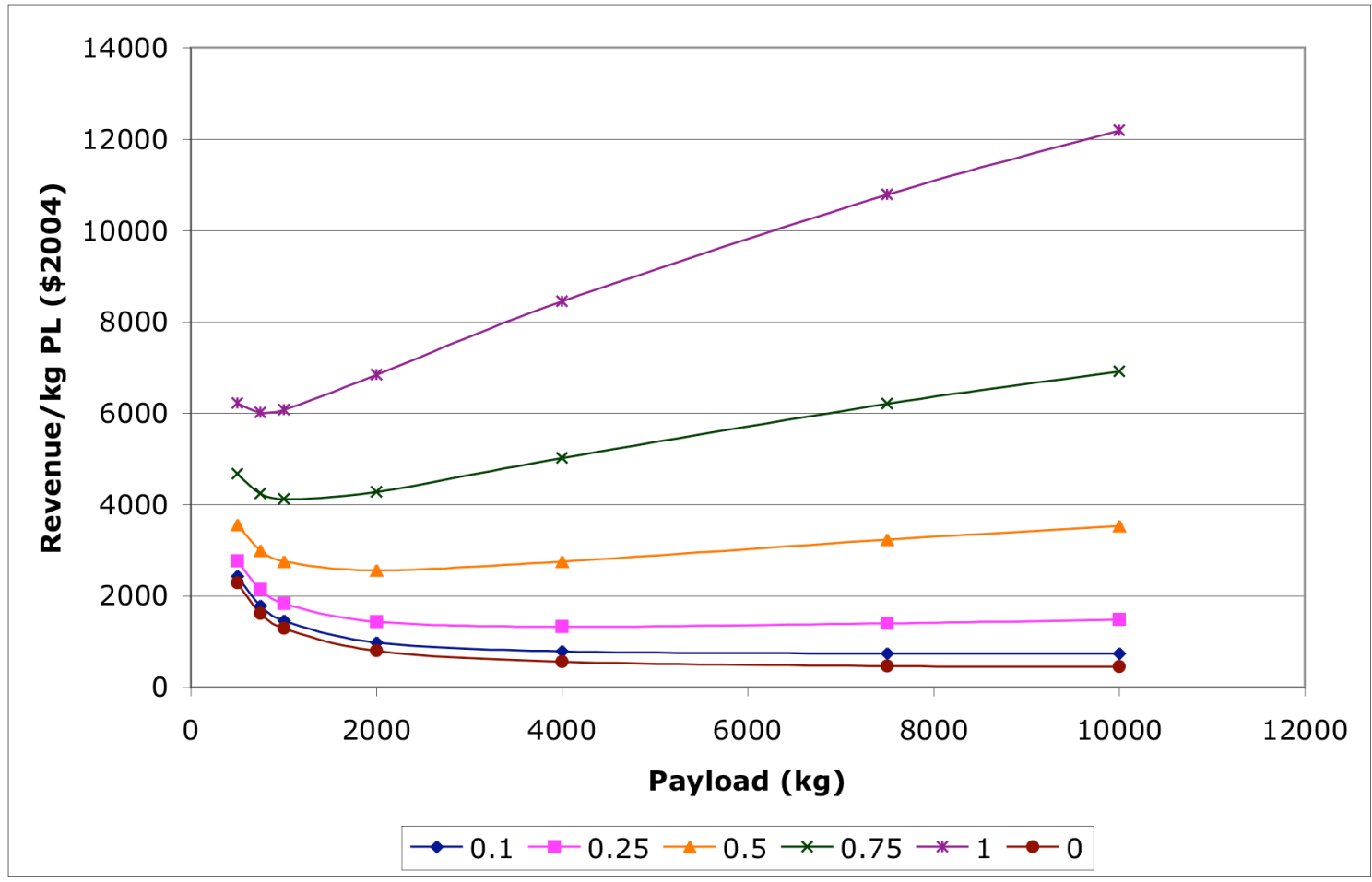

Figure 5.18: Zoomed-in view of Figure 5.17 
Figure 5.18 is the same data as Figure 5.17 but focuses on the low end of the payload range to highlight the points where cost minimums occur.

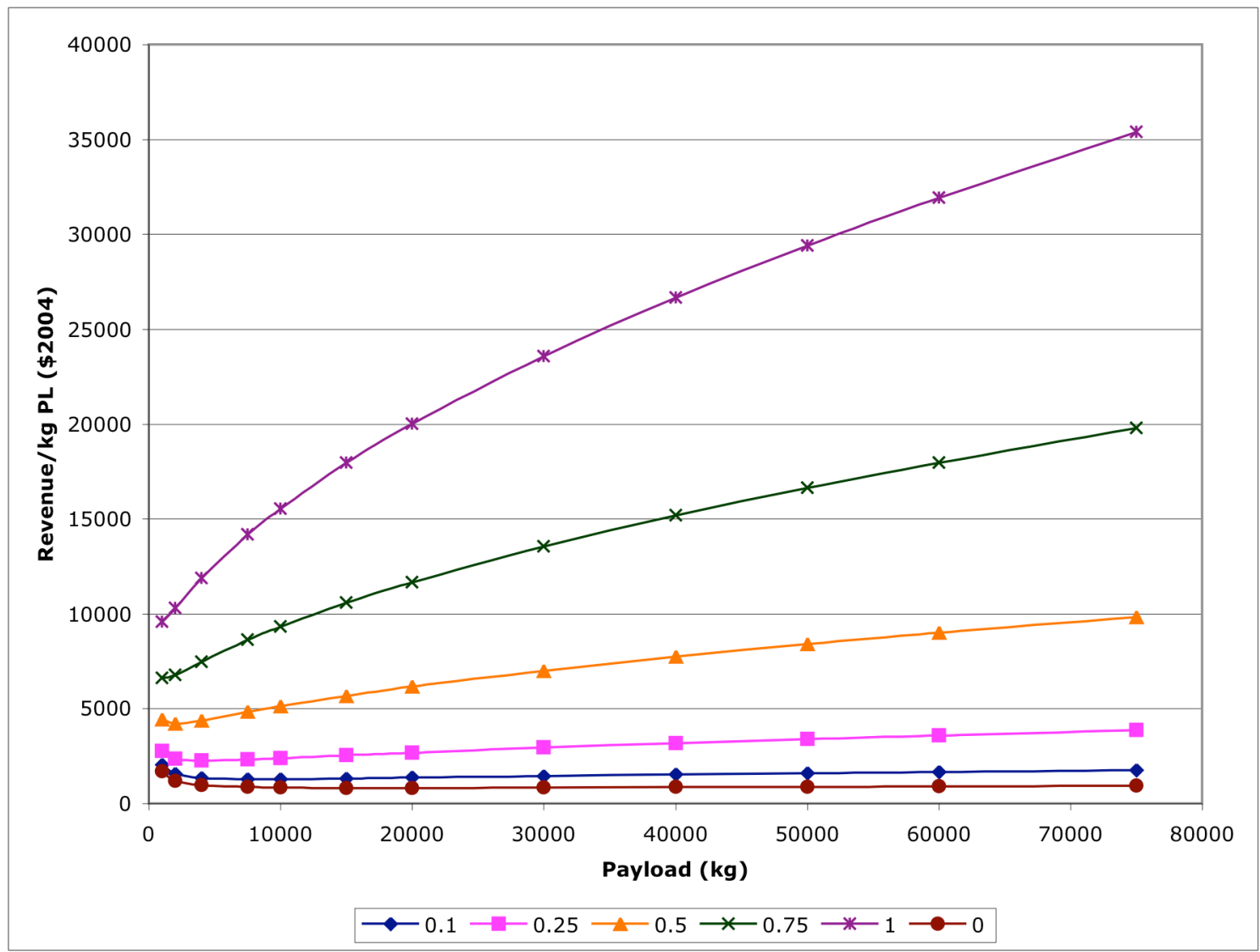

Figure 5.19: Revenue required at various rates of return for large refurbishment fraction $(0.20)$ 


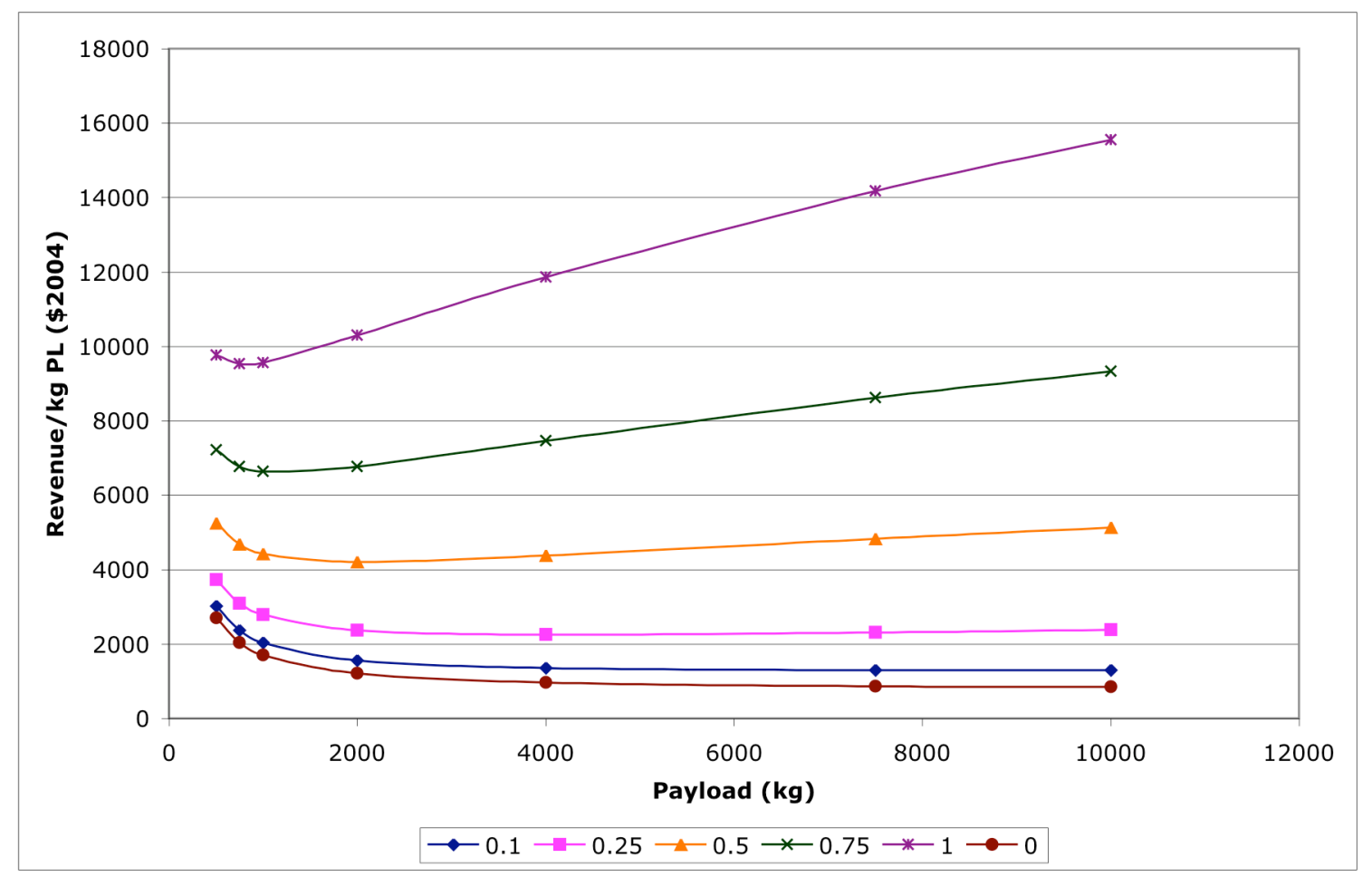

Figure 5.20: Zoomed-in view of Figure 5.19

Figure 5.20 is the same data as Figure 5.19 but focuses on the low end of the payload range to highlight the points where cost minimums occur. 


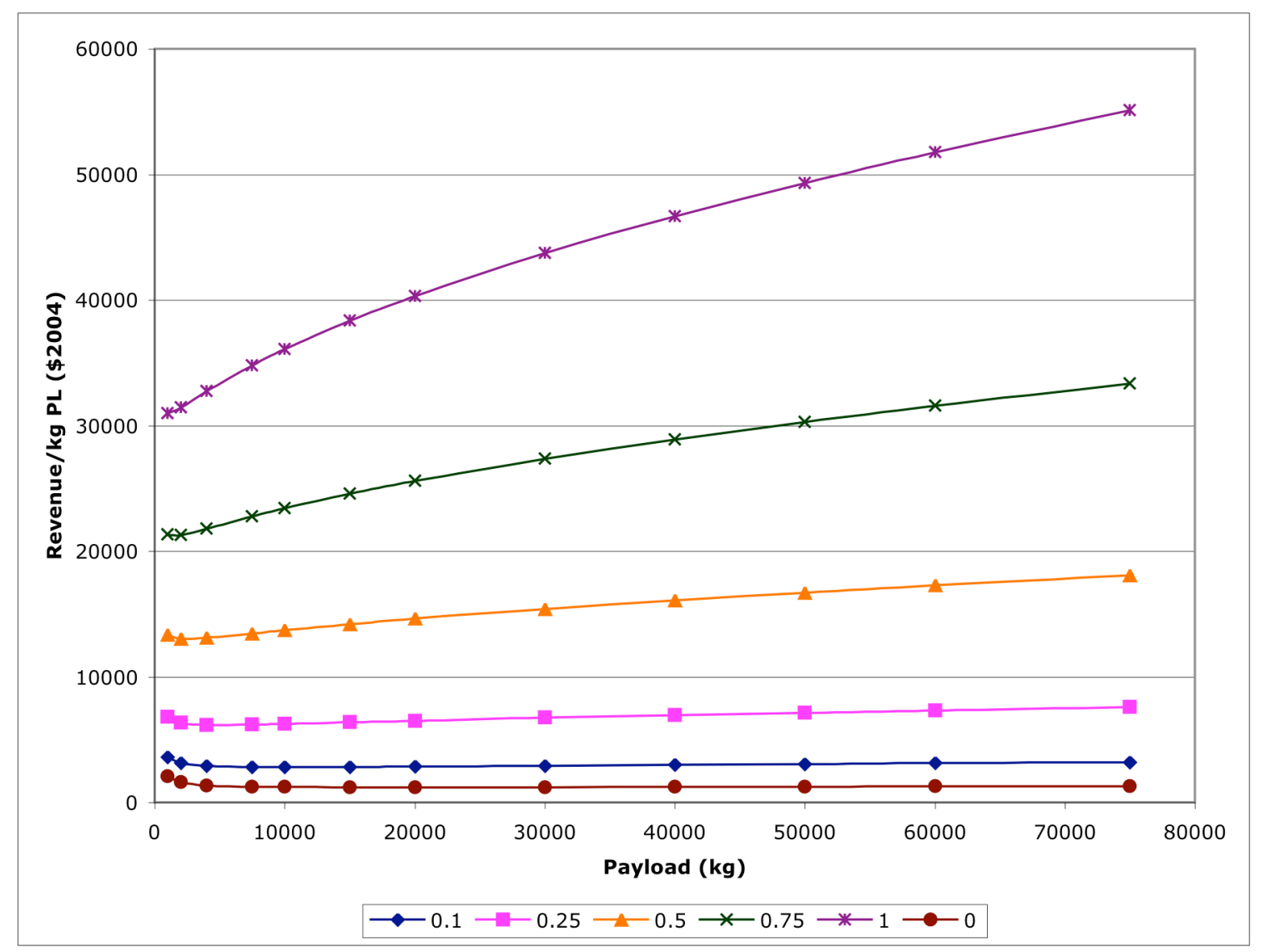

Figure 5.21: Revenue required at various rates of return for baseline ELV 


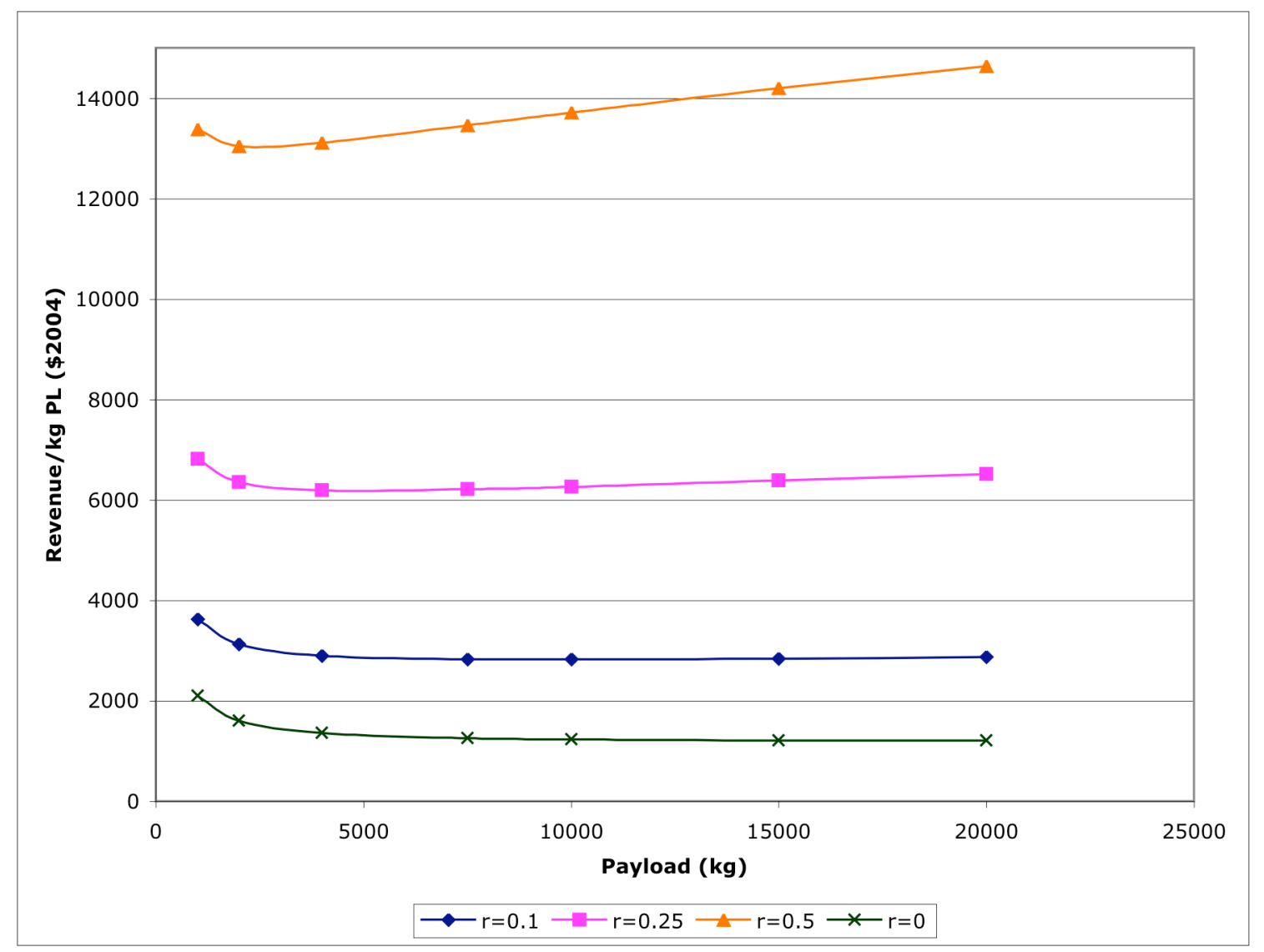

Figure 5.22: Zoomed-in view of Figure 5.21

By visual inspection of Figures 5.19-5.22 the same general trend applies at low through high levels of reusability and for the baseline expendable vehicle. The baseline expendable vehicle requires higher revenue to achieve the specified rate of return because the principal cost is higher due to the high recurring costs of expendable vehicles. For the reusable vehicles, the cost minimums occur at similar payload values regardless of the refurbishment fraction. Intuitively the costs themselves rise to higher levels at high refurbishment costs. As the required rate of return by investors increases, the payload at which a payload cost minimum occurs decreases. At low payloads the nonrecurring costs are low relative to the production and refurbishment costs. At higher payloads the nonrecurring costs become comparable to the production and refurbishment costs, so the 
nonrecurring costs savings seen at low payloads isn't a factor. Rate of return equal to 0 is a control representing no cost discounting. At a rate of return of 0 , the payload where minimum cost occurs is closer to the intermediate values noted previously. A cost advantage is seen in small payload vehicles when cost discounting is applied. A fleet of small, cheaply refurbishable, reusable launch vehicles flown at high flight rates has a cost advantage.

\subsubsection{Cost discounting and modularity}

In Chapter 5.1 modularity is shown to reduce nonrecurring costs, however a first stage divided into multiple booster units has a higher payload cost per kilogram than a monolithic first stage due to rising recurring and refurbishment costs. Modularity becomes even more important when time effects of money supplies are considered. The required revenue per kilogram payload as the first stage is broken down into modules changes similarly to the cost per kilogram payload trends shown in Chapter 5.1. At very small payloads the required revenue increases as the number first stage modules increases. Given a vehicle with a small payload size (under $10,000 \mathrm{~kg}$ ), it is not cost optimal to break a first stage into modules if cost discounting is applied. However as the payload size increases, breaking the first stage into modules causes the revenue required for breakeven given a return rate to decrease. An example is shown in Figure 5.23 below. 


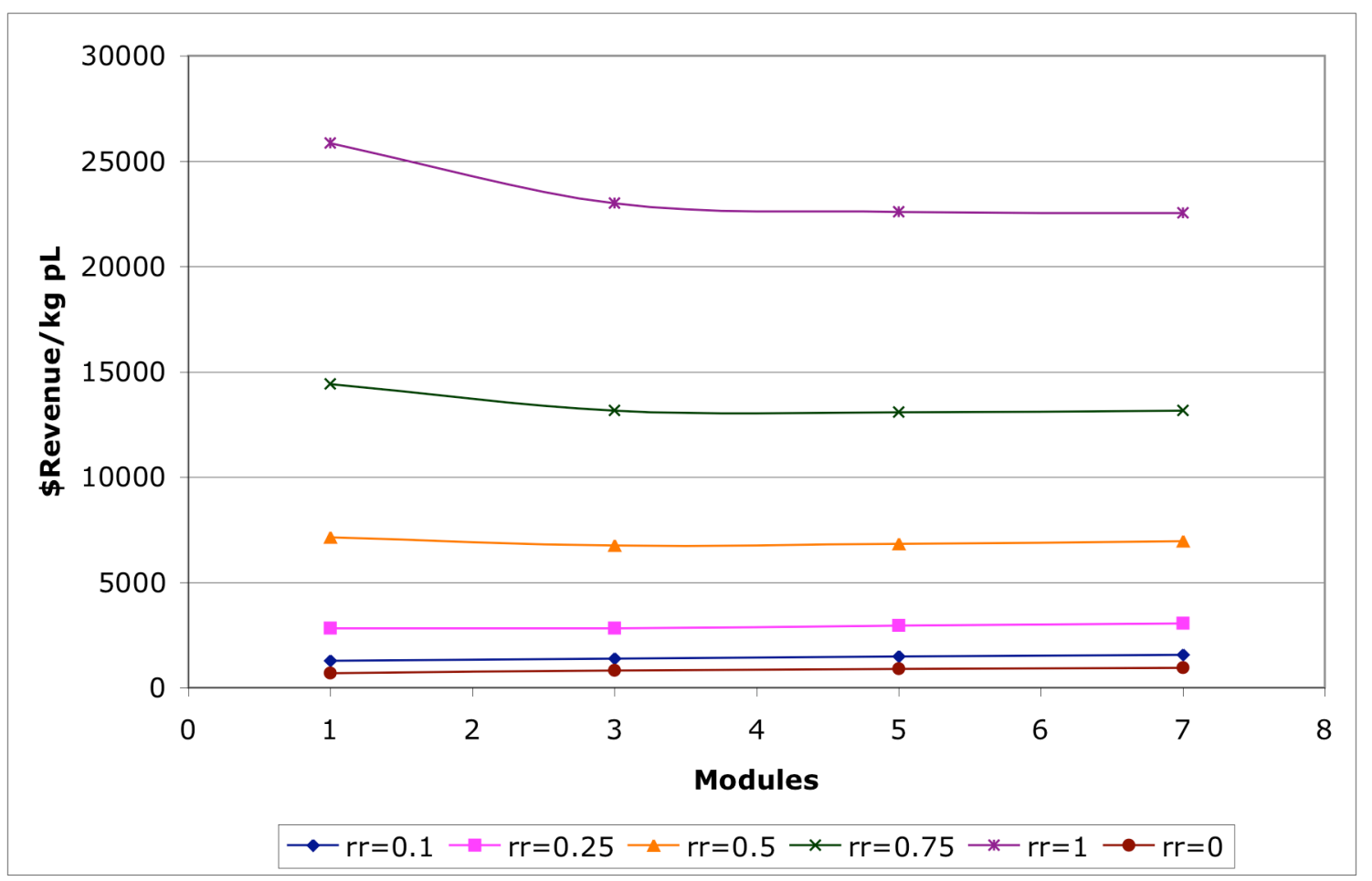

Figure 5.23: Modularity applied to discounting at $20,000 \mathrm{~kg}$ payload

Here a cheaply refurbishable reusable vehicle (refurbishment fraction equals 0.05 ) is shown with a payload of $20,000 \mathrm{~kg}$. At the high return rates illustrated in the figure, the revenue required to reach breakeven decreases as more modules are added.

Nonrecurring cost decrease as the first stage is broken up into modules. Nonrecurring costs occur during years 1-5 of this program and are the cost driver during this period. By deferring these costs to later years in the program at high return rates, the nonrecurring costs benefits are sufficient to overcome the cost increases in recurring, refurbishment and operations costs caused by multiple modules. Reducing these cost drivers over the course of the program results in reduced revenue required for breakeven at high return rates. However, as can be seen in Figure 5.23 above, these cost savings are minimal. Several key conditions, including return rate, $\Delta V$ split, inert mass fraction and the number of modules must be set within a limited range of values for modularity to 
become cost beneficial. More research should be done with modular vehicle concepts and cost discounting to fully understand its benefits and drawbacks. 


\section{Future Work and Conclusions}

\subsection{Future Work}

The results and analysis presented in this research can be expanded. One series of cost estimating relationships are used, NASA's Spacecraft/Vehicle Level Cost Model. It would be interesting to examine if using other CERs produce comparable results with regards to the relationships between refurbishment fraction and number of flights per vehicle and cost discounting and modularity.

This study assumes a launch market where the demand is inelastic. A total program payload mass of 20M kilograms must be launched regardless of the launch vehicle cost. It would be interesting to examine a marketplace where the demand is elastic. Such a market would see demand rising as prices dropped and vice versa. Large numbers of flights are necessary to examine key parameters in minimizing costs in this study. It would be noteworthy to examine more conservative launch marketplaces such as the present market of 50-70 launches a year. Certainly this would make developing and maintaining a cost effective RLV fleet a difficult obstacle.

Insurance costs include the costs of replacing a lost or failed launch vehicle and the missed opportunity costs due to not having the payload available until a later time. Wertz [9] states that the "typical insurance cost for current launch systems is on the order of $15 \%$ of the launch costs." Reusable vehicles will have very high insurance costs due to the extra operations complexity in recovering the vehicle and the unseen risks caused by testing new technology. Wertz states "reusable vehicles must be made significantly more reliable than expendable vehicles in order to be economically reliable." However with a high launch rate, a lower rate of reliability may be acceptable for unmanned 
missions given favorable insurance costs. Adding insurance costs at various levels of reliability into the model would provide additional considerations for reusable vehicles vs. their expendable counterparts in minimizing payload costs.

Finally, this study only examines unmanned ELVs and RLVs to LEO. In reality there is a requirement for vehicles, both manned and unmanned, to travel to other points in space. Geostationary orbits carry communications satellites, and lunar and interplanetary launch trajectories will be required to carry humans back to the Moon and to Mars someday. Key design parameters in determining costs of these scenarios should be identified.

\subsection{Conclusions}

Minimizing cost is an important driver in launch vehicle development. New technology must not only improve on physical design of launch vehicles (make the vehicle lighter, higher payload, more fuel efficient) but also make the vehicle more cost effective. Most launch applications in the present use ELVs; the only partially reusable vehicle in use today is the Shuttle. Reusable vehicles drive down recurring costs, which become quite costly for a launch vehicle program comprised of expendable vehicles, as an entirely new vehicle must be produced for each flight. However RLVs represent new technology and ultimately cost more to develop and refurbish. It appears that refurbishment fraction is a key parameter in determining whether it is more cost effective to recover, repair and refurbish a vehicle as opposed to building a new one. If the refurbishment costs are above $15 \%$, the analysis shows that expendable vehicles are most cost effective. Intuitively it would seem that flying a fully reusable vehicle is beneficial, 
but it turns out there is an optimum number of flights per vehicle to minimize payload costs. Beyond a certain point, the fleet size becomes so small that learning curve effects are minimal and the refurbishment costs become very high to furnish replacement parts. Essentially each replacement part becomes a hand-built part and the benefits of assembly line production are lost.

Generally, for both expendable and reusable vehicles there exists an optimum payload size for minimizing costs in the 10,000-20,000 kg range. High operations costs due to a high number of flights drive the costs up at payloads lower than this range. At payloads higher than this range, the costs are dominated by nonrecurring costs. With fewer flights per vehicle, there exists less opportunity for these nonrecurring costs to be amortized over the vehicles lifetime. This has always been a prohibitive measure in producing reusable vehicles in the commercial marketplace. It has been shown that a high flight rate is required to make a reusable vehicle more cost effective than an expendable vehicle. A high flight rate increases the opportunity for amortization of nonrecurring costs. Government and military LEO needs don't justify a flight rate high enough to make reusable vehicle sufficiently cost effective to overcome their high development costs. With more commercial entries into the market place, this could push flight rates into hundreds and thousands of flights over the lifetime of a launch vehicle program's life, giving ample opportunity for development costs to be amortized. However these high development costs that could be reduced by commercial enterprise entering into the market are exactly what scares off most commercial and private investments. A new launch vehicle is a high-risk investment that may not provide the expected rate of return. Splitting the first stage of a multiple stage launch vehicle into 
modules is one way to curb high development costs. By making smaller identical modules to form the first stage, the inert mass decreases for individual units. This causes development costs to decrease since they are a function of inert mass. With lower development costs, there is a better chance at investors seeing their expected rate of return. Also producing more identical units puts the production line further down the learning curve, thereby reducing refurbishment cost. Recurring costs and per-flight operations cost do rise for modular first stages, but the cost savings provided in nonrecurring and refurbishment costs outweigh these increases.

Based on the results of this analysis, sample vehicle architecture for a new launch vehicle fleet to launch $20 \mathrm{M} \mathrm{kg}$ to LEO in 20 years is presented here. This launch vehicle would be a reusable launch vehicle with a payload capacity of $10,000-20,000 \mathrm{~kg}$. It would be moderately cheap to refurbish at $5 \%$ of the first unit production costs. Each unit would fly 43 missions, the optimum number at a 5\% refurbishment fraction for minimizing payload costs, before being retired. At this flight rate it would take production of 25-50 vehicles to fly $1000-2000$ flights to take the required $20 \mathrm{M} \mathrm{kg}$ of payload to LEO over 20 years. The vehicle design and production processes would start 5 years and 3 years respectively before the first scheduled flight. This program would seek private and commercial investors to help foot the bill for the large number of missions required. Assuming an expected rate of return of $50 \%$ the cost per flight would be $\$ 35 \mathrm{M}-\$ 92 \mathrm{M}$ per flight or $\$ 3800-\$ 5000$ per kilogram payload launched. This would be a $65 \%-75 \%$ cost savings over flying an expendable vehicle similar to the baseline case with the same rate of return expected from investors. 


\section{Bibliography}

[1] Koelle, D. "Cost Efficiency as Design and Selection Criteria for Future Launch Vehicles", Acta Astonautica. Vol. 57, International Astronautical Federation. 2005.

[2] Closing the Business Case for Commercial Reusable Launch Vehicles. Futron Corporation. Bethesda, MD. April 2002.

[3] The Space Launch Industry Recent Trends and Near-Term Outlook. Futron Corporation. Bethesda, MD. Oct. 2004.

[4] 2006 Commercial Space Transportation Forecasts. Federal Aviation Administration Commercial Space Transportation Advisory Committee. May 2006.

[5] Koelle, D. TRANSCOST 6.0: Statistical-Analytical Model for Cost Estimation and Economical Optimization of Space Transportation Systems. TransCostSystems, Ottobrunn, Germany, 1995.

[6] Koelle, H.H., Johenning, B., TRASIM 2.0: Space Transportation Simulation Model. TU Berlin, ILR Mitt. 319(1997) 1997.

[7] NASA Cost Estimating Web Site. Spacecraft/Vehicle Level Cost Model. http://www1.jsc.nasa.gov/bu2/SVLCM.html

[8] Koelle, D. "Cost Engineering - The New Paradigm for Space Launch Vehicle Design", Journal of Reducing Space Missions Cost. Vol. 1., No. 1. Dordrecht, The Netherlands: Kluwer Academic Publishers. 1998.

[9] Wertz, James R., "Economic Model of Reusable vs. Expendable Launch Vehicles", Presented at the IAF Congress, Rio de Janeiro, Brazil, Oct. 2000.

[10] Griffin, Michael D., and Claybaugh, William R., "On the Economics of Staging for Reusable Launch Vehicles", Presented at the Space Technology and Applications International Forum, Albuquerque, NM, January 1996.

[11] Miller, R. H., "The Effect of Demand on Optimum Launch Vehicle Size" Journal of Spacecraft and Rockets, AIAA, July-August 1979.

[12] Wertz, J. R. and Larson, W. J., ed. Space Mission Analysis and Design ( $3^{\text {rd }}$ Edition). Torrance, CA and Dordrecht, The Netherlands: Microcosm Press and Kluwer Academic Publishers. 1999.

[13] Akin, D., "A Systems Analysis of Space Industrialization" ScD Dissertation, Massachusetts Institute of Technology. Aug. 1981. 
[14] U.S. Department of Labor, Bureau of Labor Statistics website. Consumer Price Index. http://www.bls.gov/cpi/home.htm 2006.

[15] Isakowitz, Steven J. International Reference Guide to Space Launch Systems. AIAA 1995.

[16] Wilhite, A.W., Engelund, W.C., Stanley, D.O., Naftel, J. C., Lepsch, R.A., Bush, L.B., Wurster, K.E., "Technology and Staging Effects on Two-Stage-to-Orbit Systems", Journal of Spacecraft and Rockets, Vol. 31, No. 1, AIAA, Feb. 1994.

[17] Wingfield III, Loran L., "Staging Evaluation of a Two-Stage-to-Orbit Vehicle at Mach 8", AIAA Paper 2001-1815, April 2001.

[18] Wade, Mark, “OTRAG”, http://www.astronautix.com/lvs/otrag.htm 1997-2006.

[19] David, Leonard, "NASA Shuts Down X33, X34 Programs", http://www.space.com/missionlaunches/missions/x33_cancel_010301.html March 2001. 